\title{
A statistical analysis of the elastic distortion and dislocation density fields in deformed crystals
}

\author{
Mamdouh S. Mohamed ${ }^{\mathrm{a}, 1,{ }^{*}}$, Ben C. Larson ${ }^{\mathrm{b}}$, Jon Z. Tischler $^{\mathrm{c}}$, and Anter El-Azab ${ }^{\mathrm{d}}$ \\ ${ }^{a}$ Department of Scientific Computing, Florida State University, Tallahassee, FL 32306, USA \\ Email: mamdouh.s.mohamed@gmail.com \\ ${ }^{\mathrm{b}}$ Materials Sciences and Technology Division, Oak Ridge National Laboratory, Oak Ridge, TN 37831, USA \\ Email: larsonbc@ornl.gov \\ ${ }^{\mathrm{c}}$ X-ray Sciences Division, Advanced Photon Source, Argonne National Laboratory, Argonne, IL 60439, USA \\ Email: tischler@aps.anl.gov \\ ${ }^{\mathrm{d}}$ School of Nuclear Engineering and School of Materials Engineering, Purdue University, West Lafayette, IN 47907, \\ USA \\ E-mail: aelazab@purdue.edu \\ Phone: +1 7654966864
}

* Corresponding Author: Mamdouh S. Mohamed, Physical Sciences and Engineering Division, KAUST, Thuwal, Jeddah 23955, KSA, Phone (Cell): +966561510040, (work): +966128080863

${ }^{1}$ Present address: Physical Sciences and Engineering Division, KAUST, Thuwal, Jeddah 23955, KSA 


\begin{abstract}
.
The statistical properties of the elastic distortion fields of dislocations in deforming crystals are investigated using the method of discrete dislocation dynamics to simulate dislocation structures and dislocation density evolution under tensile loading. Probability distribution functions (PDF) and pair correlation functions $(\mathrm{PCF})$ of the simulated internal elastic strains and lattice rotations are generated for tensile strain levels up to $0.85 \%$. The PDFs of simulated lattice rotation are compared with sub-micrometer resolution three-dimensional X-ray microscopy measurements of rotation magnitudes and deformation length scales in $1.0 \%$ and $2.3 \%$ compression strained $\mathrm{Cu}$ single crystals to explore the linkage between experiment and the theoretical analysis. The statistical properties of the deformation simulations are analyzed through determinations of the Nye and Kröner dislocation density tensors. The significance of the magnitudes and the length scales of the elastic strain and the rotation parts of dislocation density tensors are demonstrated, and their relevance to understanding the fundamental aspects of deformation is discussed.
\end{abstract}

Keywords: Discrete dislocation dynamics, dislocation density tensor, lattice rotation, 3D X-ray microscopy 


\section{Introduction}

\section{Metal plasticity is carried out by dislocations moving under the influence of applied stresses and}

their mutual interactions in crystals. During the course of plastic deformation, the density of dislocations increases significantly and the dislocations themselves tend to aggregate into highly heterogeneous structures. Associated with these dislocation structures are complex internal stress and elastic distortion fields. The heterogeneity of these fields gives rise to strain hardening in deformed metals and represents a driving force for grain refinement and post deformation recrystallization. Accordingly, investigating the internal elastic fields and their gradients in deforming crystals is important to understanding both the deformation and post deformation properties of metals.

Based on the dislocation-induced elastic distortion fields, a key measure of the crystal incompatibility due to dislocations is defined in terms of the dislocation density tensor. This tensor was initially introduced by Nye (1953) as a quantitative characterization of the elastic lattice rotation gradients in deformed crystals. Later, Kröner (1981) incorporated the elastic strain into the definition and established the dislocation density tensor as a central quantity in his continuum theory of dislocations. The importance of the dislocation density tensor has become apparent in plasticity theories both as a primary field in continuum dislocation dynamics (Acharya, 2001; Limkumnerd and Sethna, 2007) and as a definition for geometrically necessary dislocation (GND) densities in strain gradient plasticity theories (Gao et al., 1999; Huang et al., 2000; Gao and Huang, 2003). The inclusion of the dislocation density tensor in plasticity models has motivated experimental measurements through the electron backscattering diffraction (EBSD) technique and x-ray microbeam diffraction. The EBSD technique has provided a means 
to determine the Nye dislocation density tensor in two dimensions (i.e., crystal surfaces, serial sectioning) through measurements of local lattice rotation fields. For example, lattice rotation measurements were used to study the evolution of GND densities near grain boundaries in deformed aluminum crystals (Sun et al., 2000; El-Dasher et al., 2003) and polycrystalline copper (Jiang et al., 2013). Moreover, by incorporating focused ion beam serial sectioning, EBSD was extended to three dimensions (Zaefferer et al., 2008) to study the GND density distributions and indentation size effects for copper crystals (Zaafarani et al., 2008; Demir et al., 2009). The elastic strain contribution to the GND density is often not addressed experimentally, especially in the absence of a quantitative assessment of the significance of the elastic strain contribution to the dislocation density tensor. However, it is significant that in addition to lattice rotation field measurements, advances in the EBSD technique (Wilkinson et al., 2006; Villert et al., 2009; Kacher et al., 2009) have enabled the detection of surface elastic strain fields and the determination of the Kröner dislocation density tensor at surfaces (Wilkinson and Randman, 2010; Britton et al., 2013; Wilkinson et al. 2014).

The development of submicron resolution three-dimensional x-ray microscopy (3DXM) has provided the capability to perform nondestructive spatially resolved measurements of both local lattice orientations and elastic strain fields in 3D over mesoscopic length scales (Larson et al., 2002; Yang et al., 2003; Levine et al., 2006; Larson and Levine, 2013). Nondestructive measurements of the Nye dislocation density tensor were reported for a (2D) cross-sectional plane (Larson et al., 2008) in elastically and plastically bent silicon plates, demonstrating that the 2D dislocation density tensor for an elastically bent Si plate vanishes and that the Nye tensor for a plastically deformed Si plate contains GND traces predicted for thin plate plastic deformation (Cleveringa et al., 1999). 
At present, high spatial resolution, high angular resolution, nondestructive experimental measurements of local elastic strains and local rotations in deformed materials over mesoscopic length scales of tens of micrometers is in progress. However, as experimental capabilities mature they will provide the possibility of direct, quantitative, non-destructive comparisons between experiments and discrete dislocation dynamics predictions (Devincre et al., 2011; Ghoniem et al., 2000; Arsenlis et al., 2007). Of course, such direct, one-to-one comparisons between theoretical predictions and experimental measurements for stochastic processes such as dislocation generation and evolution can be performed quantitatively only through quantitative, statistical based analyses of simulations and experimental measurements.

This communication presents a statistical theoretical analysis of discrete dislocation dynamics based simulations of the elastic distortion fields and the dislocation density tensor in a $\sim 5 \mu \mathrm{m}$ cube of $\mathrm{Cu}$ undergoing tensile strains up to $0.85 \%$. An example of $\mathrm{x}$-ray measurements of lattice rotation in lightly deformed $\mathrm{Cu}$ is presented to underscore the motivation of the method and provide a reference for comparison of the predicted distortion fields with measurements. The theoretical basis for calculating the elastic strain, lattice rotation, and dislocation density tensor is presented in section 2. In section 3, the statistical measures used in the analysis are defined, followed by the dislocation dynamics simulation conditions and the numerical implementation in section 4. Section 5 presents and discusses the physical implications of the results of dislocation dynamics simulations of deformation in $\mathrm{Cu}$ along with a preliminary comparison with mesoscale 3D x-ray microscopy measurements of deformation-induced lattice rotations in $\mathrm{Cu}$. Section 6 discusses the outlook for combined simulation and measurement investigations of the fundamental aspects of deformation in ductile crystalline materials.

\section{Theoretical formalism for the elastic fields in a deformed crystal}


In order to provide a self-contained presentation, we review briefly the theoretical formalism for crystal deformation, denoting the elastic displacement field of a dislocation line by $u_{i}$. Within the linear theory of elasticity, the gradient of $u_{i}\left([\nabla \mathbf{u}]_{i j}=\partial_{i} u_{j}\right)$ is decomposed into symmetric and skew-symmetric parts, defining the elastic strain $\varepsilon_{i j}$ and lattice rotation $w_{i j}$ fields, respectively, following the Cartesian tensor notation. This additive decomposition is expressed in the form

$$
\begin{aligned}
& \partial_{i} u_{j}=\varepsilon_{i j}+w_{i j}, \\
& \varepsilon_{i j}=\frac{1}{2}\left(\partial_{i} u_{j}+\partial_{j} u_{i}\right), \\
& w_{i j}=\frac{1}{2}\left(\partial_{i} u_{j}-\partial_{j} u_{i}\right) .
\end{aligned}
$$

where $\partial_{i}=\partial / \partial x_{i}$. The rotation tensor $w_{i j}$ is represented in terms of its axial vector $\omega_{k}=\left(e_{i j k} w_{i j}\right) / 2$, where $e_{i j k}$ is the permutation symbol. For the purpose of computing the elastic fields of a crystal undergoing a statistically homogeneous deformation, we consider a representative volume element (RVE), $\Omega$, containing a statistically large number of dislocations embedded in an infinite crystal. Within this RVE, the internal elastic fields are found by the superposition of two contributions, one contribution due to dislocations inside the RVE itself and a second due to dislocations outside the RVE. The former contribution is given by the infinitemedium solution of all dislocations within the RVE while the latter is the image field (Hirth and Lothe, 1982). The elastic strain and rotation fields are expressed in the form

$$
\begin{aligned}
& \varepsilon_{i j}=\varepsilon_{i j}^{\infty}+\varepsilon_{i j}^{I}, \\
& w_{i j}=w_{i j}^{\infty}+w_{i j}^{I},
\end{aligned}
$$


with the superscripts $\infty$ and $I$ referring to the infinite domain and image fields, respectively. The former fields are computed assuming that dislocations are embedded in an infinite elastic medium. Adopting the regularized approximation (Cai et al., 2006) to avoid singularities in the classical expressions, the infinite medium contribution to the elastic strain and lattice rotation tensors are expressed in the form:

$$
\begin{aligned}
& \varepsilon_{m l}^{\infty}(\mathbf{x})=\frac{-1}{16 \pi} \oint_{L} b_{p}\left[\left(\delta_{k m} e_{p q l}+\delta_{k l} e_{p q m}-2 \delta_{l m} e_{p q k}\right) \partial_{q} \nabla^{2} R_{a}+\frac{1}{1-v} e_{p j k} \partial_{l} \partial_{j} \partial_{m} R_{a}\right] d x_{k}^{\prime}, \\
& w_{m l}^{\infty}(\mathbf{x})=\frac{-1}{16 \pi} \oint_{L}\left[e_{l i k}\left(b_{i} \partial_{m}+b_{m} \partial_{i}\right)+e_{i m k}\left(b_{l} \partial_{i}+b_{i} \partial_{l}\right)\right] \nabla^{2} R_{a} d x_{k}^{\prime},
\end{aligned}
$$

where $\partial_{i}=\partial / \partial x_{i}, R_{a}=\left(R^{2}+a^{2}\right)^{1 / 2}$, with $R=\left(\Delta x_{i} \Delta x_{i}\right)^{1 / 2}, \Delta x_{i}=\left(x_{i}^{\prime}-x_{i}\right), a$ is a non-singularity parameter, $v$ is the Poisson's ratio and $\delta_{i j}$ is the Kronecker delta (Deng et al., 2008). For an RVE that has dislocations piercing its boundary, the image contribution to the elastic distortion fields is calculated by solving a traction boundary value problem (BVP) developed by Deng et al. (2008). The BVP for the image stress field, $\sigma_{i j}^{I}$, is stated as follows:

$\partial_{j} \sigma_{i j}^{I}+F_{i}=0, \quad F_{i}=\partial_{k} \sigma_{i k}^{\infty}$ in $\Omega$ $n_{j} \sigma_{i j}^{I}=T_{i}, \quad T_{i}=-n_{k} \sigma_{i k}^{\infty}$ on $\partial \Omega$

where $\sigma_{i k}^{\infty}$ is the infinite-domain stress of dislocations inside the RVE $\Omega, n_{k}$ is the normal to its boundary, and $F_{i}$ and $T_{i}$ are the body forces within $\Omega$ and the traction on $\partial \Omega$, respectively. The stress equilibrium equations above are supplemented by Hooke's law and the strain-displacement relationship, and a Galerkin finite element method is used to solve the BVP. The finite element scheme finds the image displacement field, which is then used to determine image the 
contribution to the elastic strain and rotation tensors. The latter are subsequently added to the infinite-domain contributions given in Eqs. (3) and (4) to determine the total fields. The elastic distortion fields are then used to determine the dislocation density tensor by differentiation.

In the continuum dislocation theory (Kröner, 1981), the deformed state of a crystal is reached by first introducing an inhomogeneous plastic distortion, $\beta_{i j}^{p}$, to form a non-compact intermediate configuration, followed by imposing an elastic distortion, $\beta_{i j}^{e}$, to bring the intermediate configuration into the final state. The total distortion is thus given by

$\beta=\beta_{i j}^{p}+\beta_{i j}^{e}$

Since both the initial and final states are compact, the total distortion $\beta_{i j}$ is compatible and thus can be expressed as the gradient of a displacement field $\tilde{u}_{i}$, i.e., $\beta_{i j}=\partial_{i} \tilde{u}_{j}$, which implies that $\beta_{i j}$ is irrotational, or

$e_{i k l} \partial_{k} \beta_{l j}=e_{i k l} \partial_{k} \partial_{l} \tilde{u}_{j}=0$

Eqs. (6) and (7) lead to Kröner's definition of the dislocation density tensor $\boldsymbol{\alpha}_{i j}$ (Kröner, 1981),

$\alpha_{i j}=e_{i k l} \partial_{k} \beta_{l j}^{p}=-e_{i k l} \partial_{k} \beta_{l j}^{e}$

We note that $\tilde{u}_{j}$ is a compatible displacement field while the elastic displacement field $u_{i}$ introduced above Eq. (1) suffers a closure failure when integrated over a closed path encircling a dislocation or a set of dislocations with non-trivial Burgers vectors. After some manipulations, 
the dislocation density tensor can be defined as a function of the elastic strain and lattice rotation by

$\alpha_{i j}=\partial_{j} \omega_{i}-\delta_{i j} \partial_{k} \omega_{k}-e_{i k l} \partial_{k} \varepsilon_{l j}$

where $\omega_{i}$ is an axial rotation vector (Kröner, 1981). For negligibly small spatial variations of the elastic strain field, the above definition of the Kröner dislocation density tensor reduces to that introduced originally by Nye (1953),

$\alpha_{i j}^{\mathrm{Nye}}=\partial_{j} \omega_{i}-\delta_{i j} \partial_{k} \omega_{k}$

Considering the discrete nature of dislocations in the crystal, the dislocation density tensor averaged over a volume element $V_{e}$ can also be expressed in terms of the dislocation segments contained in the element as follows, (Nye, 1953):

$\alpha_{i j}=\frac{-1}{V_{e}} \sum_{m=1}^{M} t_{i}^{m} b_{j}^{m}$,

where $M$ is the number of segments in $V_{e}$ and $t_{i}^{m}$ is the line direction of dislocation segment $m$. The difference between Eq. (11) and the original formula developed by Nye (1953) (i.e. the negative sign and the implied tensor transpose) is due to the different convention used to define the gradient of the elastic displacement vector and the curl of the displacement gradient tensor, where the convention adopted be Kröner (1981) was followed here in defining the dislocation density tensor. Eq. (11) provides a means to validate the computational scheme fixing the dislocation density tensor using Eq. (9). The difference between the predicted $\alpha_{i j}$ values by both equations is discussed in section 5.4. 


\section{Statistical description of internal elastic fields}

The internal elastic fields in a deforming crystal are generated by the dislocation configuration in the crystal. The statistical properties of these fields are thus linked to the statistics of the underlying dislocation system. Here, we define the statistical measures for an internal tensor field $\eta_{i j}$, e.g., elastic strain, lattice rotation, or dislocation densities by referring to a probability density function of the dislocation system itself (Deng, 2008). In the spirit of dislocation dynamics, we consider a dislocation system that is discretized into a large number $n$ of small segments of a given configuration. Considering many such possible configurations, one may be able to describe them using a joint probability density function of the system of segments. Let the $k$-th segment belong to a given slip system $s s_{k}$ and have a position vector $\mathbf{x}_{k}$ and a line tangent described by one angle $\theta_{k}$ measured relative to a fixed direction, say the slip direction, in its slip plane. Here, $\mathbf{x}_{k} \in \mathfrak{R}^{3}$ and $\theta_{k} \in \Theta$, with $\mathfrak{R}^{3}$ and $\Theta$ being the 3D Cartesian space and the periodic angular space, $[0,2 \pi)$, respectively. We note that the coordinates $\mathbf{x}_{k}$ and $\theta_{k}$ are random but the dislocation systems is properly connected. The $n$-th order (joint) probability density function (PDF) $f^{\left(s s_{1}, \ldots, s_{n}\right)}\left(\mathbf{x}_{1}, \theta_{1}, \ldots, \mathbf{x}_{n}, \theta_{n}\right)$ for the dislocation system is defined such that

$\int f^{\left(s s_{1}, \ldots, s s_{n}\right)}\left(\mathbf{x}_{1}, \theta_{1}, \ldots, \mathbf{x}_{n}, \theta_{n}\right) d \mathbf{x}_{1} d \theta_{1} \ldots d \mathbf{x}_{n} d \theta_{n}=1$

with $f^{\left(s s_{1}, \ldots, s s_{n}\right)}\left(\mathbf{x}_{1}, \theta_{1}, \ldots, \mathbf{x}_{n}, \theta_{n}\right) d \mathbf{x}_{1} d \theta_{1} \ldots d \mathbf{x}_{n} d \theta_{n}$ being the probability of finding segment 1 in the spatial volume $d \mathbf{x}_{1}$ and orientation range $d \theta_{1}$ at $\mathbf{x}_{1}$ and $\theta_{1}, \ldots$, and segment $n$ in the spatial volume $d \mathbf{x}_{n}$ and orientation range $d \theta_{n}$ at $\mathbf{x}_{n}$ and $\theta_{n}$. For the sake of defining the joint probability above, the segment size can be made as small as needed, in which case the coordinate 
$\mathbf{x}_{k}$ refers to, say, the center point of the segment. The probability of finding a segment in the spatial volume $d \mathbf{x}_{1}$ and orientation range $d \theta_{1}$ at $\mathbf{x}_{1}$ and $\theta_{1}$ can be obtained by integrating over all coordinates and angles except $\mathbf{x}_{1}$ and $\theta_{1}$.

The first order PDF of $\eta_{i j}$ generated by the dislocations is defined by

$$
p_{i j}\left(\eta_{\circ} ; \mathbf{x}\right)=\int f^{\left(s s_{1}, \ldots, s s_{n}\right)}\left(\mathbf{x}_{1}, \theta_{1}, \ldots, \mathbf{x}_{n}, \theta_{n}\right) \delta\left(\eta_{\circ}-\eta_{i j}(\mathbf{x})\right) d \mathbf{x}_{1} d \theta_{1} \ldots d \mathbf{x}_{n} d \theta_{n}
$$

where the field $\eta_{i j}(\mathbf{x})=\eta_{i j}\left(\mathbf{x} ; \mathbf{x}_{1}, \theta_{1}, \ldots, \mathbf{x}_{n}, \theta_{n}\right)$ is generated at $\mathbf{x}$ by the dislocation configuration at hand. The role of the Dirac-delta in Eq. (13) is to reduce the integration over the phase space $\Gamma=\left(\mathfrak{R}^{3} \times \Theta\right)^{n}$ to the subset of this space in which $\eta_{i j}(\mathbf{x})=\eta_{\circ}$. The Dirac-delta in Eq. (13) has the inverse units of its argument and, as a result, $p_{i j}\left(\eta_{\circ} ; \mathbf{x}\right)$ is a probability density function in the $\eta_{i j}$-space and it is defined such that $p_{i j}\left(\eta_{\circ} ; \mathbf{x}\right) d \eta_{\circ}$ is the probability of finding $\eta_{i j}$ at point $\mathbf{x}$ in the interval $d \eta_{\circ}$ at $\eta_{\circ}$. Under the condition of statistical homogeneity, the ensemble average of an internal field is equal to its volume average; this statement enables us to define a positionindependent first order PDF of the form

$p_{i j}\left(\eta_{\circ}\right)=\frac{1}{\Omega} \int_{\Omega} p_{i j}\left(\eta_{\circ} ; \mathbf{x}\right) d \mathbf{x}$,

where $\Omega=\int d \mathbf{x}$ is the volume of an RVE in an homogeneously deformed crystal.

The second order PDF $p_{i j k l}\left(\eta_{\circ}, \eta_{\circ}^{\prime} ; \mathbf{x}, \mathbf{x}^{\prime}\right)$ of the tensor field $\eta_{i j}$ is similarly defined by

$$
\begin{aligned}
& p_{i j k l}\left(\eta_{\circ}, \eta_{\circ}^{\prime} ; \mathbf{x}, \mathbf{x}^{\prime}\right)= \\
& \quad \int f^{\left(s s_{1}, \ldots, s s_{n}\right)}\left(\mathbf{x}_{1}, \theta_{1}, \ldots, \mathbf{x}_{n}, \theta_{n}\right) \delta\left(\eta_{\circ}-\eta_{i j}(\mathbf{x})\right) \delta\left(\eta_{\circ}^{\prime}-\eta_{k l}\left(\mathbf{x}^{\prime}\right)\right) d \mathbf{x}_{1} d \theta_{1} \ldots d \mathbf{x}_{n} d \theta_{n},
\end{aligned}
$$


which is defined such that $p_{i j k l}\left(\eta_{\circ}, \eta_{\circ}^{\prime} ; \mathbf{x}, \mathbf{x}^{\prime}\right) d \eta_{\circ} d \eta_{\circ}^{\prime}$ is the joint probability of finding $\eta_{i j}(\mathbf{x})$ in the range $d \eta_{\circ}$ at $\eta_{\circ}$ and $\eta_{k l}\left(\mathbf{x}^{\prime}\right)$ in $d \eta_{\circ}^{\prime}$ at $\eta_{\circ}^{\prime}$. The pair correlation function (PCF) $\operatorname{cor}_{i j k l}\left(\eta_{\circ}, \eta_{\circ}^{\prime} ; \mathbf{x}, \mathbf{x}^{\prime}\right)$ is defined in terms of the first and second order PDFs as follows:

$$
\operatorname{cor}_{i j k l}\left(\eta_{\circ}, \eta_{\circ}^{\prime} ; \mathbf{x}, \mathbf{x}^{\prime}\right)=\frac{p_{i j k l}\left(\eta_{\circ}, \eta_{\circ}^{\prime} ; \mathbf{x}, \mathbf{x}^{\prime}\right)}{p_{i j}\left(\eta_{\circ} ; \mathbf{x}\right) p_{k l}\left(\eta_{\circ}^{\prime} ; \mathbf{x}^{\prime}\right)}
$$

The spatial dependence is the property of interest in characterizing crystal deformation. As such a different PCF is defined by integrating the corresponding PDFs over $\eta_{\circ}$ and $\eta_{\circ}^{\prime}$. This yields

$$
\operatorname{cor}_{i j k l}\left(\mathbf{x}, \mathbf{x}^{\prime}\right)=\frac{\iint \eta_{\circ} \eta_{\circ}^{\prime} p_{i j k l}\left(\eta_{\circ}, \eta_{\circ}^{\prime} ; \mathbf{x}^{\prime} \mathbf{x}^{\prime}\right) d \eta_{\circ} d \eta_{\circ}^{\prime}}{\int \eta_{\circ} p_{i j}\left(\eta_{\circ} ; \mathbf{x}\right) d \eta_{\circ} \int \eta_{\circ}^{\prime} p_{k l}\left(\eta_{\circ}^{\prime} ; \mathbf{x}^{\prime}\right) d \eta_{\circ}^{\prime}}=\frac{\left\langle\eta_{i j}(\mathbf{x}) \eta_{k l}\left(\mathbf{x}^{\prime}\right)\right\rangle}{\left\langle\eta_{i j}(\mathbf{x})\right\rangle\left\langle\eta_{k l}\left(\mathbf{x}^{\prime}\right)\right\rangle}
$$

The angle brackets above refer to an ensemble average which, in our case, is assumed to be equal to the volume average. Expressing $\eta_{i j}$ as $\eta_{i j}=\left\langle\eta_{i j}\right\rangle+\delta \eta_{i j}$ and $\eta_{k l}$ in the same way, with $\delta \eta_{i j}$ denoting the fluctuation about $\left\langle\eta_{i j}\right\rangle$, the above correlation can be recast in the form

$$
\operatorname{cor}_{i j k l}\left(\mathbf{x}, \mathbf{x}^{\prime}\right)=1+\frac{\left\langle\delta \eta_{i j}(\mathbf{x}) \delta \eta_{k l}\left(\mathbf{x}^{\prime}\right)\right\rangle}{\left\langle\eta_{i j}(\mathbf{x})\right\rangle\left\langle\eta_{k l}\left(\mathbf{x}^{\prime}\right)\right\rangle}
$$

For internal stresses or elastic strain fields, the above PCF forms are problematic because the ensemble averages are typically zero. To avoid this computational indeterminacy, a correlation expression of the following form is introduced, 


$$
\begin{aligned}
\operatorname{cor}_{i j k l}\left(\mathbf{x}, \mathbf{x}^{\prime}\right) & =\frac{\operatorname{cov}\left(\eta_{i j}(\mathbf{x}), \eta_{k l}\left(\mathbf{x}^{\prime}\right)\right)}{\left[\operatorname{var}\left(\eta_{i j}(\mathbf{x})\right) \operatorname{var}\left(\eta_{k l}\left(\mathbf{x}^{\prime}\right)\right)\right]^{1 / 2}} \\
& =\frac{\left\langle\eta_{i j}(\mathbf{x}) \eta_{k l}\left(\mathbf{x}^{\prime}\right)\right\rangle-\left\langle\eta_{i j}(\mathbf{x})\right\rangle\left\langle\eta_{k l}\left(\mathbf{x}^{\prime}\right)\right\rangle}{\left[\left\langle\eta_{i j}^{2}(\mathbf{x})\right\rangle-\left\langle\eta_{i j}(\mathbf{x})\right\rangle^{2}\right]^{1 / 2}\left[\left\langle\eta_{k l}^{2}\left(\mathbf{x}^{\prime}\right)\right\rangle-\left\langle\eta_{k l}\left(\mathbf{x}^{\prime}\right)\right\rangle^{2}\right]^{1 / 2}}
\end{aligned}
$$

where $\operatorname{cov}(.,$.$) and \operatorname{var}($.$) are the covariance and variance of the enclosed quantities. Since the$ mean values, $\left\langle\eta_{i j}(\mathbf{x})\right\rangle$ and $\left\langle\eta_{k l}\left(\mathbf{x}^{\prime}\right)\right\rangle$ are identically zero in the case of strain and lattice rotation, the last expression simplifies to

$$
\operatorname{cor}_{i j k l}\left(\mathbf{x}, \mathbf{x}^{\prime}\right)=\frac{\left\langle\eta_{i j}(\mathbf{x}) \eta_{k l}\left(\mathbf{x}^{\prime}\right)\right\rangle}{\left\langle\eta_{i j}^{2}(\mathbf{x})\right\rangle^{1 / 2}\left\langle\eta_{k l}^{2}\left(\mathbf{x}^{\prime}\right)\right\rangle^{1 / 2}}=\frac{\left\langle\delta \eta_{i j}(\mathbf{x}) \delta \eta_{k l}\left(\mathbf{x}^{\prime}\right)\right\rangle}{\left\langle\eta_{i j}^{2}(\mathbf{x})\right\rangle^{1 / 2}\left\langle\eta_{k l}^{2}\left(\mathbf{x}^{\prime}\right)\right\rangle^{1 / 2}}
$$

For a statistically homogeneous deformation state and a sufficiently large RVE, all correlations become functions of coordinate difference by the principle of translation invariance. This means that $\operatorname{cor}_{i j k l}\left(\mathbf{x}, \mathbf{x}^{\prime}\right)=\operatorname{cor}_{i j k l}\left(\mathbf{x}^{\prime}-\mathbf{x}\right)=\operatorname{cor}_{i j k l}(\Delta \mathbf{x})$. We further specify a radial PCF $C_{i j k l}(r)$ as a simple version of $\operatorname{cor}_{i j k l}(\Delta \mathbf{x})$, defined in terms of $r=|\Delta \mathbf{x}|$ by $C_{i j k l}(r)=\operatorname{cor}_{i j k l}(|\Delta \mathbf{x}|)$. This expression implies that the radial PCF is always in the range $[-1,1]$, where a positive PCF value means that $\eta_{i j}(0)$ and $\eta_{k l}(r)$ are on the same side of the average field value and a negative value means that they are on opposite sides of the average value. The analyses in this paper focus only on the self PCF, $C_{i j i j}(r)$, which is evaluated using expression (20) along with the above simplification. The decaying part of $C_{i j i j}(r)$ will be fit to an exponential form $C_{i j i j}(r)=C(r)=A \exp \left(-r / \ell_{0}\right)$, with $\ell_{0}$ being a correlation length.

\section{Numerical simulations}


Numerical simulations of deformation were performed using the Discrete Dislocation Dynamics (DDD) library microMegas (Devincre et al., 2011). A simulated copper single crystal of size 4.6 $\times 5.0 \times 5.4 \mu \mathrm{m}$ was loaded in tension under the constraint of a constant strain rate of $25 \mathrm{~s}^{-1}$ along the [001] direction. Copper has 12 primary slip systems of type $a / 2\langle 110)\{111\}$, where the $<110>$ Burgers vectors have magnitudes $b=2.553 \AA$, and the nominally $5 \mu \mathrm{m}$ edge length cube was seeded with an initial dislocation density of $1.6 \times 10^{12} \mathrm{~m} / \mathrm{m}^{3}$ in the form of closed loops distributed randomly in space over the 12 slip systems. Tensile deformation simulations were performed up to a total strain of $0.85 \%$, during which the dislocation density increased by more than an order of magnitude to $1.8 \times 10^{13} \mathrm{~m} / \mathrm{m}^{3}$. In addition to analyzing the dislocation distribution for $0.85 \%$ strain, dislocation configurations were collected at intermediate strain levels in the range $0.15 \%-0.85 \%$, for which the elastic strain and lattice rotation fields were computed as well. To solve the BVP (5), the simulation volume was partitioned into 8-node brick elements of edge size 0.1 micrometers. Numerical integration of the body forces inside each volume element and the traction forces on each surface element was implemented using 4 quadrature points in each direction. The elastic strain and lattice rotation tensors were calculated at the center of each volume element as an average over the 4 sample points in each direction. The Nye and Kröner dislocation density tensors were then calculated for each element using the elastic fields at the element and at its neighboring elements using Eq. (9) and Eq. (10). Since periodic boundary conditions were applied during the simulation, the dislocation system and the resulting elastic fields are assumed to be statistically homogeneous over an infinite crystal. Accordingly, the PDFs and PCFs and their radially averaged form $C_{i j i j}(r)$ were computed using Eq. (14) and Eq. (20), respectively. To avoid edge effects in the results (Deng and El-Azab, 2007), the absolute 
value of the separations of Cartesian coordinates $\Delta x, \Delta y$ and $\Delta z$ were confined to values of less than $0.75 L$ (where $L$ is the nominally $5 \mu \mathrm{m}$ cube edge length) during the PCF calculations.

\section{Results and discussion}

\subsection{Statistical properties of deformation induced elastic strain}

The probability distribution function (PDF) for the elastic strain tensor in the crystal for $0.85 \%$ strain is displayed in Fig. 1(a). The shear strain components exhibit nearly symmetric distributions around a mean value of zero while the normal strain distributions exhibit slightly skewed distributions. Analysis of the corresponding stress PDF in previous work (Deng, 2008) showed that both shear and normal stress components exhibit symmetric PDFs. The fact that the shear strain components are proportional to the stress counterparts makes the PDF of the shear strain components symmetric. However, because each normal strain component is a linear superposition of three normal stress components, and because the stress signs differ for various stress components at a particular point in space, symmetry of normal strain PDF is not guaranteed. The observed skewness of the normal strain PDF does not affect the average values of the strain components, which were found to be zero. Moreover, analysis of the elastic strain PDF for the intermediate strain levels (not shown) showed that the widths of the distributions of the strain components increased and the peak heights decreased with increasing the strain level.

The radial self-correlations $C_{i j i j}(r)$ at the maximum strain of $0.85 \%$ are displayed in Fig. 1(b). The correlations decay from unity at $r=0$ to a no-correlation value of 0 at $r \approx 1.5$ micrometers; the initial rapid falloff in the correlation is followed by weak oscillations corresponding to weakly correlated and anti-correlated behavior that is increasingly damped at larger distances and higher deformation levels. A similar behavior was reported previously for dislocation 
density self-correlations (Deng and El-Azab, 2007), where the oscillatory behavior in the dislocation correlations was interpreted as evidence for the emergence of dislocation patterns. We note that oscillations in the elastic strain correlations are related to the spatial characteristics of the underlying dislocation systems, especially dislocation density heterogeneities. Since elastic strains are integrally connected with the underlying dislocation system, discussion of PCF patterning will be deferred to sections 5.2 and 5.4, where the correlation lengths for the elastic strain are compared with lattice rotation and the dislocation density tensor correlations. As will be emphasized later, the elastic strain PCFs better represent the spatial features of the underlying dislocation structure than the rotation field PCFs.

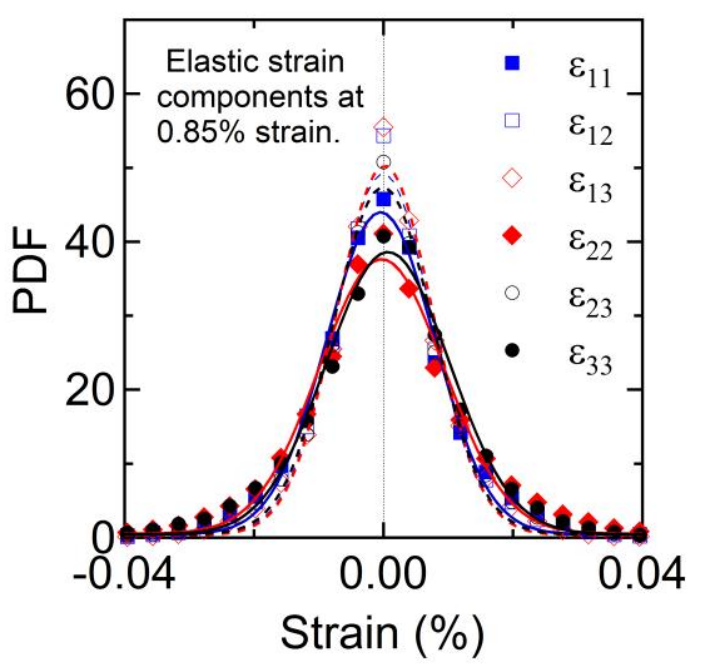

(a)

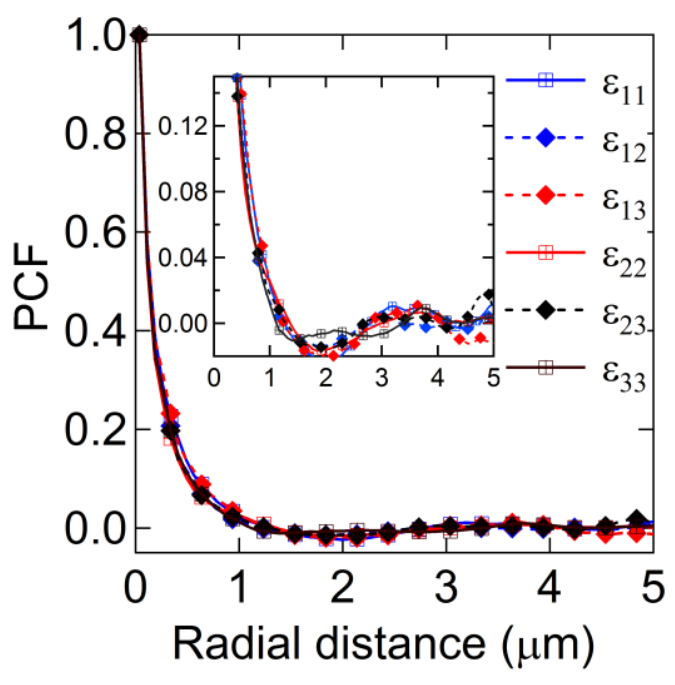

(b)

Fig.1. (a) The probability density function of the elastic strain components at a tensile strain value of $0.85 \%$; the PDFs are fit to Gaussian distributions. (b) Radial self-correlation of the elastic strain at a tensile strain value of $0.85 \%$. The inset shows an amplified view of the correlation demonstrating an oscillation at long range.

\subsection{Statistical properties of deformation induced lattice rotations}

Figs. 2(a) and (b) show the distribution of the simulated lattice rotation components at strain levels of $0.25 \%$ and $0.85 \%$, respectively. All components exhibit nearly symmetric distributions 
with only a small amount of skewness in the central part of the distribution. A comparison between Fig. 2(a) (at strain level of $0.25 \%$ ) and Fig. 2(b) (at strain level of $0.85 \%$ ) reveals a strong similarity in the shapes of the probability distribution functions of the rotation fields. The rotation distribution becomes wider with lower peak height as a result of the increase in the dislocation density with increasing tensile strain. Quantitatively, the full widths at half maxima (FWHM) increase sub-linearly from $0.02^{\circ}$ at $0.25 \%$ tensile strain to approximately $0.035^{\circ}$ for $0.85 \%$ tensile strain in Fig. 2(b).

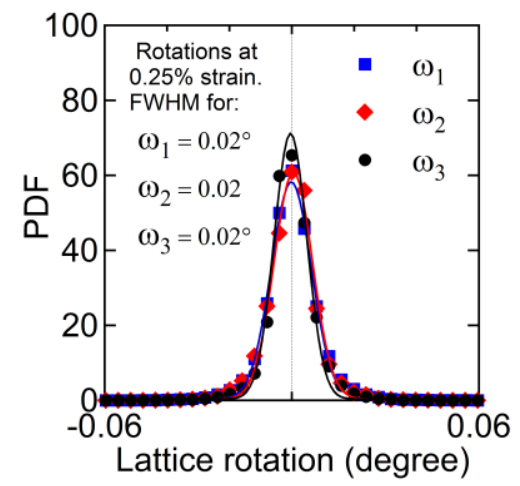

(a)

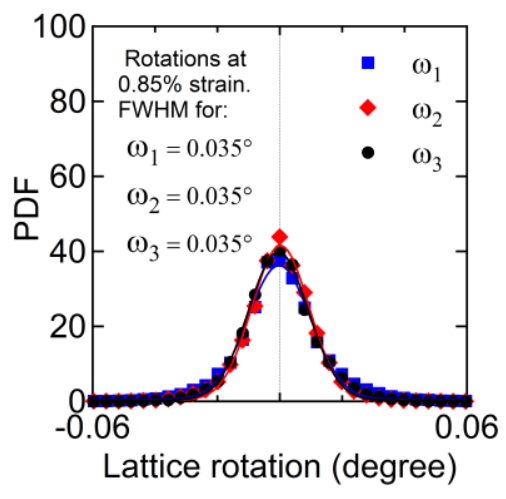

(b)

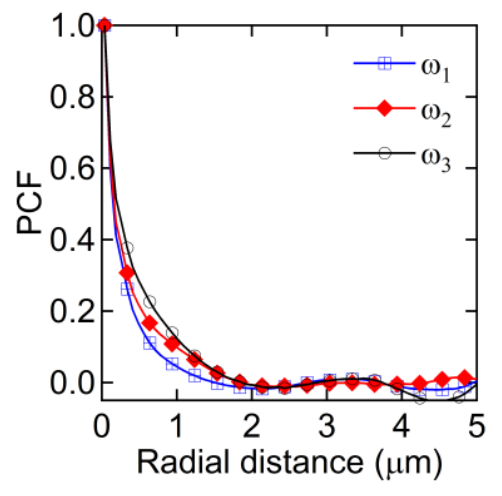

(c)

Fig.2. (a) and (b) The PDF for lattice rotations at crystal strain levels of $0.25 \%$ and $0.85 \%$, respectively. The PDFs are fit to Gaussian distributions. (c) The radial self-correlation function for the lattice rotations at a crystal strain of $0.85 \%$.

The radial self-correlations for lattice rotations shown in Fig. 2(c) reveal similarities with the strain correlations shown in Fig. 1(b) in terms of the initial decay and subsequent oscillatory behavior. However, we note that the lattice rotation correlations decay more slowly than the elastic strain correlations. The lattice rotation correlation length $\ell_{0}$ was found to fall in the range 0.40-0.57 $\mu \mathrm{m}$ compared to $0.21-0.24 \mu \mathrm{m}$ for the strain correlations at the same deformation level. In order to investigate the source of this difference, 2D maps of the elastic strain and lattice rotation fields have been inspected. Fig. 3 shows a typical map in the $y-z$ plane for $\varepsilon_{12}$ and $\omega_{3}$, 
both calculated using the dislocation configuration corresponding to the $0.85 \%$ strain case. As shown in the figure the spatial distributions of the elastic strain and rotation fields differ significantly. The differences in the length scales of the fields are not limited to the $\varepsilon_{12}$ and $\omega_{3}$ components only, but rather are observed for other components of the elastic strain and the lattice rotation fields as well. Such a behavior can be attributed to the more frequent sign reversal of the elastic strain components in comparison with the lattice rotation. On average, this makes the numerator of the correlation expression (20), which sums the products of fields at pairs of points, smaller at shorter distances causing the correlation to decay earlier. This effect can be observed in Fig. 3, where the areas across which the elastic strain component $\varepsilon_{12}$ changes its sign (i.e. red to blue) in Fig. 3(a) (i.e. dislocation-populated zones) exhibit patterns different from the lattice rotation component $\omega_{3}$ in Fig. 3(b). Larger zones, with single dominating rotation orientations, are observed more commonly for the lattice rotation components $\omega_{3}$; this ultimately results in a slower decay for the lattice rotation correlations as observed in Fig. 2(c).

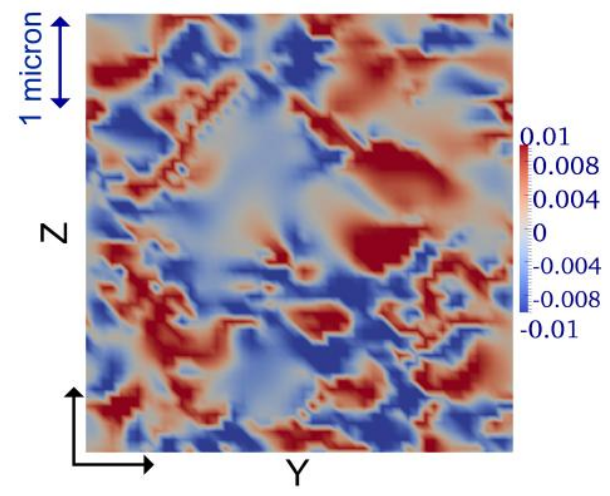

(a)

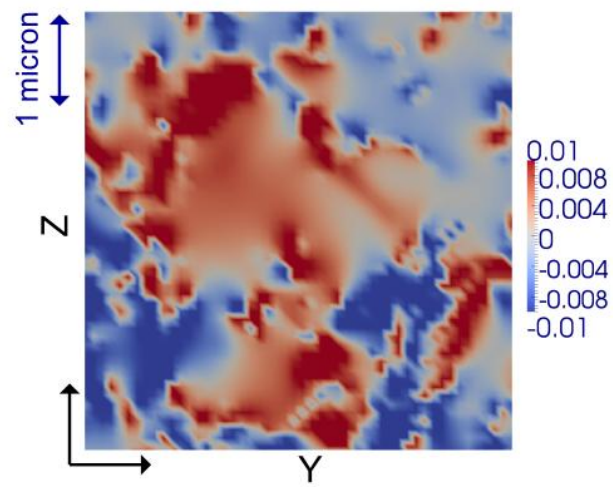

(b)

Fig. 3. 2D maps of (a) $\varepsilon_{12}(\%)$ and (b) $\omega_{3}$ (degree) in a $y$ (horizontal)- $z$ (vertical) plane in the middle of the simulated volume at a strain level of $0.85 \%$; the maps show clear differences in the spatial changes of the elastic strain and lattice rotation fields in deformed crystals.

\subsection{Experimental measurements of deformation induced lattice rotations}


Before presenting simulations of the dislocation density tensor associated with the elastic strain and the lattice rotation simulations presented in Sections 5.1 and 5.2 above, we provide spatially resolved experimentally measured lattice rotations for (initially) essentially perfect (i.e. $<10^{9}$ $\mathrm{m} / \mathrm{m}^{3}$ ) Cu single crystal that were deformed in compression to 1.0 and $2.3 \%$ for comparison with the lattice rotations simulated for 0.15 to $0.85 \%$ tensile strain in an $\sim 5 \mu \mathrm{m}$ cube. Figure 4 shows submicron resolution 3D x-ray microscopy measurements (3DXM) of local lattice rotations performed over $\sim 30 \times 40 \mu \mathrm{m}^{2}$ (parallelogram shaped) 2D slices within $\mathrm{Cu}$ crystals that had been deformed to $1.0 \%$ and $2.3 \%$ in compression along the [001] direction. These measured lattice rotation values refer to deformation induced rotations of the $\mathrm{Cu}$ lattice relative to the average orientation in the slices as a reference. The measurements were performed (nondestructively) with 0.5 micron in-plane and out of plane spatial resolution and with $\sim 0.01$ degree angular resolution over the 45-degree parallelogram shaped slices using the Sector 34 ID-E white-beam Laue 3DXM facility at the Advanced Photon Source at Argonne National Laboratory.

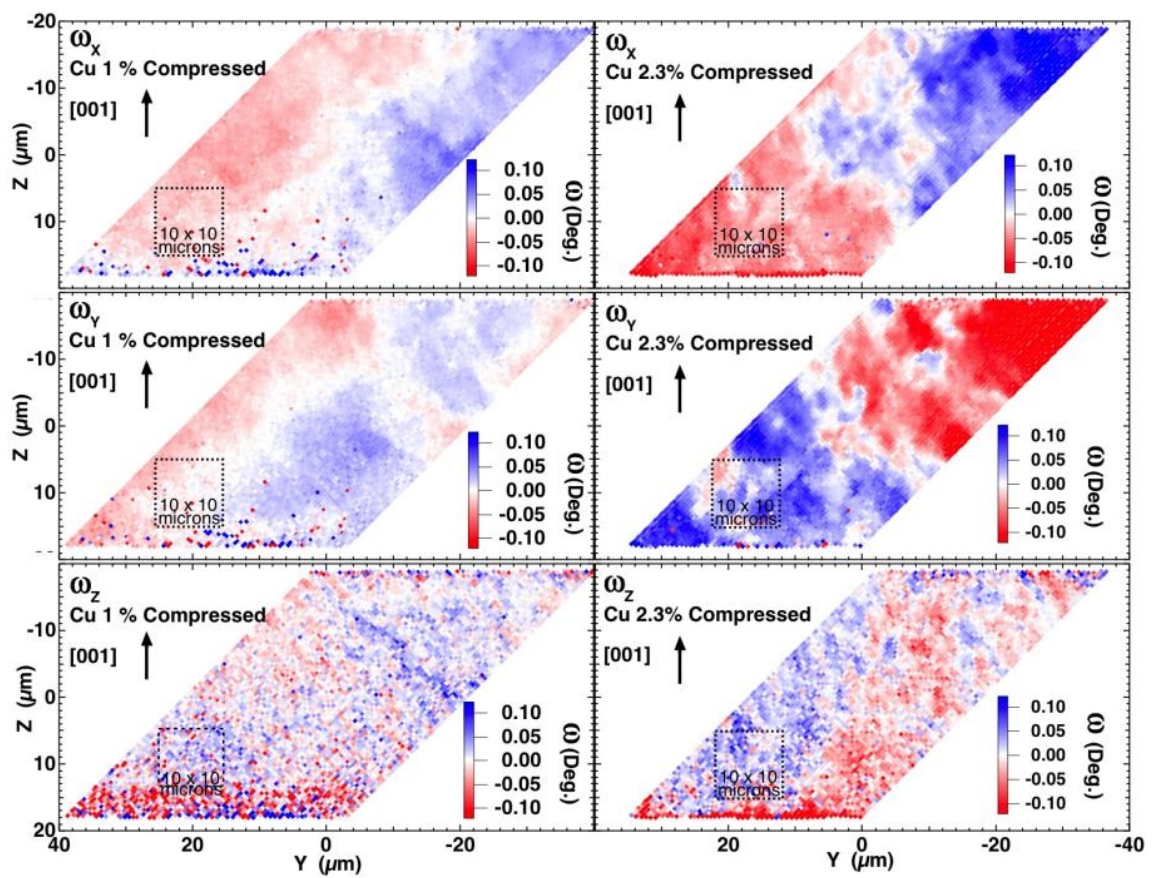


Fig. 4: Two-dimensional false-color plots of lattice rotation around the $x, y$ and $z$-axes in a $1.0 \%$ (left column) and $2.3 \%$ (right column) compressed copper single crystal.

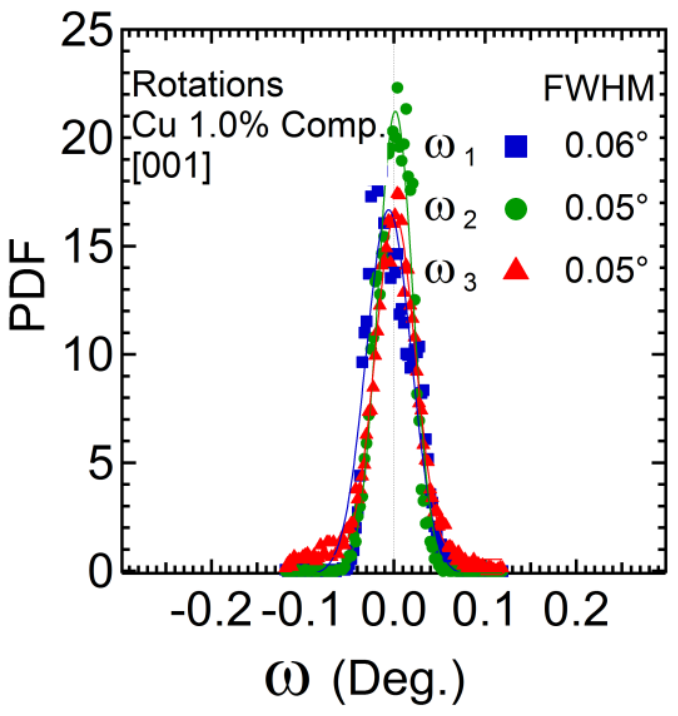

(a)

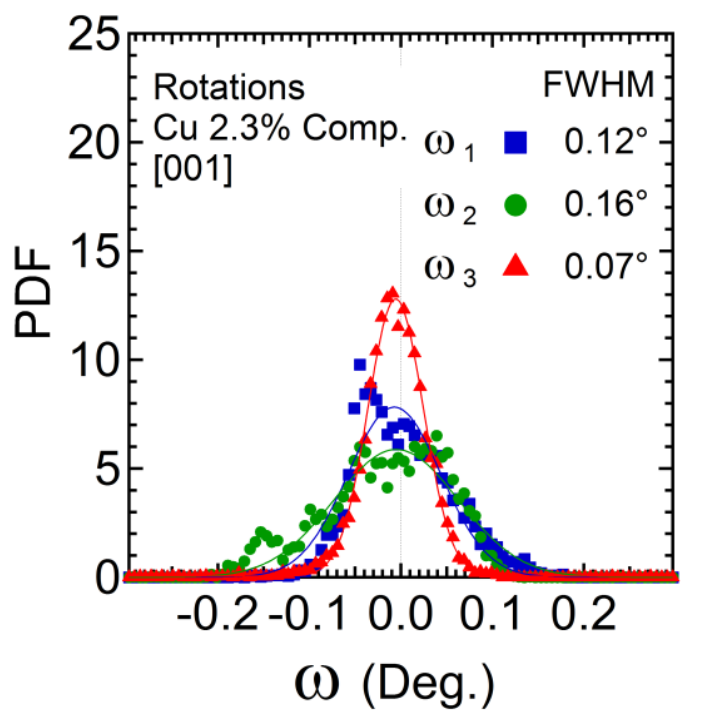

(b)

Fig.5. Probability density function of the measured lattice rotation for (a) $1.0 \%$, and (b) $2.3 \%$ compressed $\mathrm{Cu}$ single crystals. The rotation vector components $\omega_{1}, \omega_{2}$ and $\omega_{3}$ refer to the rotations around $x, y$ and $z$-axes displayed in Fig. 4. The PDFs are fit to Gaussian distributions.

Full crystallographic orientations (relative to the reference) of each $0.5 \times 0.5 \times 0.5 \mu \mathrm{m}^{3}$ voxel were determined using two Laue reflections from white beam Laue diffraction patterns. In turn, the axial vector rotations of individual voxels from the reference orientation (see Section 2 above) were decomposed into rotation components $\omega_{1}, \omega_{2}$ and $\omega_{3}$ along the $x, y$ and $z$ coordinate axes.

Accordingly, the 2D false-color plots in Fig. 4 refer to the lattice rotational components about the $x, y$ and $z$ axes, where the $\mathrm{z}$ axis is along the [001] direction of the uniaxial compression. The color scales ( $0 \pm 0.12$ degrees from full blue to full red) are the same for the $1.0 \%$ and $2.3 \%$ compressed $\mathrm{Cu}$, indicating by the levels of color saturation that the magnitudes of the rotations increase with increasing compression. The plots show further that early stage deformation is a 
multi-scale phenomenon on mesoscopic length scales over several $10 \mathrm{~s}$ of micrometers. In general, the rotations in the $1.0 \%$ compressed $\mathrm{Cu}$ are less than \pm 0.12 degrees while the $2.3 \%$ compressed $\mathrm{Cu}$ contains rotations ranging greater than \pm 0.12 degrees as indicated by the presence of areas with full color saturation. The rotation distributions are shown quantitatively as PDFs in Figs. 5 (a) and (b), where we note that for $1.0 \%$ compression the FWHM are $\sim 0.05$ degrees with very few voxels outside \pm 0.10 degrees, while even the average FWHM for $2.3 \%$ compression exceeds 0.1 degrees. The existence of predominantly red regions and predominantly blue regions on the right and left sides of the $1 \%$ plots and at the top and bottom of the $2.3 \%$ plots indicate the presence of (a very small) long-range curvature over the $\sim 30-40$ micrometer measurement ranges. This is presumably the result of very small experimental misalignments in the compression considering that the starting material was an essentially perfect crystal, but these trends could be the signature of even longer length scale deformation features as well.

Qualitative comparison between the simulated lattice rotation PDFs in Fig. 2 and the experimental rotation PDF measurements in Fig. 5 indicates similar trends for the distributions as a function of strain magnitude, where the distributions become wider with lower peaks as strain level increases. Again, this is attributed to the increase in the dislocation density with crystal deformation. In addition, some skewness is observed in the central peak position of the rotation PDFs for both simulations and experiments. Quantitatively, the FWHM for the distributions are $\sim 0.035$ degrees for the simulated rotations (at strain level of $0.85 \%$ ) and $\sim 0.05$ degrees for the experimental rotations (at a strain level of $1.0 \%$ ). Although relatively close in magnitude, the larger FWHM for the experiment compared to the simulations can be attributed in part to the impact of the long range (30-40 micron) rotation zones observed in the experimental data (Fig. 4); these could not be captured in simulations for a $\sim 5$ micrometer cube, of course. 
The quantitative and qualitative similarities exhibited by the measured and simulated rotation statistics underscore the importance of further investigations both experimentally and computationally. Although the measurements reported in Fig. 4 provide only 2D maps of the lattice rotation fields, this 3DXM technique can be extended to generate 3D maps by repeating the measurements on adjacent parallel planes within the sample (Larson et al., 2007, Larson et al., 2008). The increasingly powerful capabilities to perform nondestructive 3D spatially resolved measurements of microstructural details in deformed materials provides a strong motivation to extend dislocation dynamics simulations to larger strains, larger length scales, and more comprehensive theoretical treatments. However, the present comparisons stand as an initial quantification of the differences between the actual deformation microstructure in deformed crystals and discrete dislocation dynamics simulations. As such, they provide an initial bridge between simulations and experiment, albeit not quite overlapping in strain magnitudes.

\subsection{Statistical properties of the simulated dislocation density tensor}

As was explained in section 2, two methods can be used to calculate the dislocation density tensor for a given dislocation configuration. The first is through the elastic strain and rotation fields using Eq. (9) and the second is directly from the discrete dislocation configuration using Eq. (11). Although the latter method is straightforward, the former method is adopted in the current analysis since during experiments the dislocation density tensor is calculated from the measured elastic distortion fields. Therefore, Eq. (11) provides an appropriate means to verify the computational scheme used to calculate the dislocations density tensor using Eq. (9).

Figs. 6 (a) and (b) show 2D maps for the dislocation density tensor norm, $\left(\alpha_{i j} \alpha_{i j}\right)^{1 / 2}$, calculated using Eq. (11) and Eq. (9), respectively. The figures reveal similar spatial characteristics in 
general with minor differences. In the dislocation-free zones, the dislocation density norm calculated using Eq. (11) is identically zero. However, when using Eq. (9), small nonzero values of the tensor norm are observed. On the other hand, in dislocation-populated zones, the field peaks are seen to be slightly diffuse when computed using Eq. (9). We recall that in the calculation of the elastic distortion fields and the dislocation density tensor, the simulated domain was partitioned into cubic elements with edge sizes of 0.1 micrometers. Calculating $\alpha_{i j}$ using Eq. (11) amounts to finding the tensor components first exactly (by the virtue of having all dislocations explicitly from dislocation dynamics) and then averaging $\alpha_{i j}$ over the volume element. However, when using Eq. (9), the elastic distortion fields are first averaged over each volume element (to emulate experimental situations) and the $\alpha_{i j}$ components is then calculated by taking the derivative of those averaged elastic distortion fields numerically via a central difference scheme. The difference between the two numerical schemes therefore explains the observed difference between Figs. 6 (a) and (b). An additional contribution to this difference can be the volume over which the $\alpha_{i j}$ tensor is calculated when using the respective equations. Using Eq. (11) to calculate $\alpha_{i j}$ considers only the discrete dislocation segments falling inside the small volume element under consideration. However, for Eq. (9), $\alpha_{i j}$ is determined in a particular volume element using the elastic distortion fields calculated at the volume element and also its neighbors. Therefore, computing $\alpha_{i j}$ using Eq. (9) effectively averages the tensor value over larger volume elements. In any case, considering the apparent similarities between Figs 6 (a) and (b), we remark that Eq. (9) and Eq. (11) provide consistent descriptions for the dislocation density tensor, where the comparison between them reflects the sensitivity of $\alpha_{i j}$ to the volume over which it is calculated; i.e. mesh size effects. As mentioned earlier in this section, we will 
use Eq. (9) to calculate $\alpha_{i j}$ during the forthcoming analysis due to its consistency with experimental deformation characterizations.

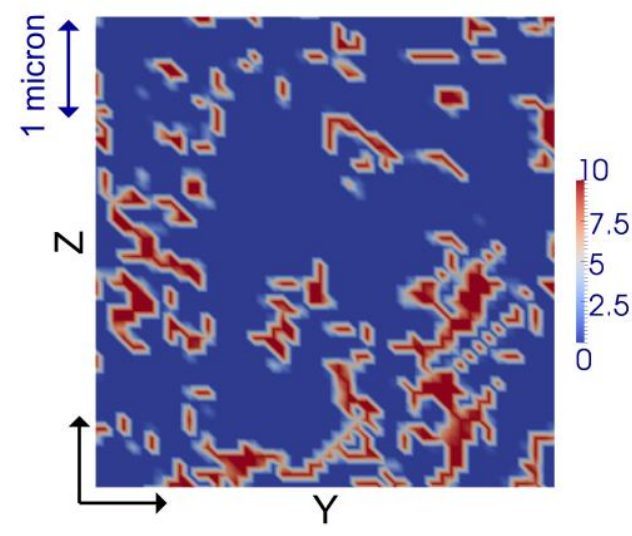

(a)

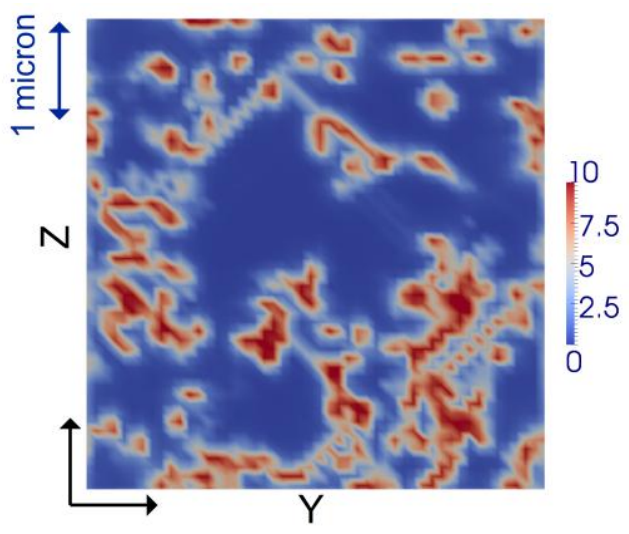

(b)

Fig. 6. A display of the norm of the dislocation density tensor ( $\mathrm{mrad} / \mathrm{micron})$ on a $y-z$ plane in the simulation volume at a strain level of $0.85 \%$ (a) calculated from the discrete dislocation configuration using Eq. (11) and (b) from the elastic distortion fields using Eq. (9).

The PDF of the components of the dislocation density tensor as defined by Kröner in Eq. (9) is shown in Fig. 7(a). It is observed that all tensor components have similar distributions; they are all nearly symmetric around a mean value of zero, indicating the predominance of small distortions. The zero mean value of the distribution reflects the nominally zero net Burgers vector and is consistent with the fact that the initial configuration for the dislocation dynamics simulation consisted of set of closed square loops and the use of periodic boundary conditions throughout the simulations. The evolution of the distributions with increasing strain levels reflects the increase of the dislocation density with strain. Actually, the PDF peaks for the tensor components at the $0.25 \%$ strain level (not shown) were higher, by almost double, in comparison to the $0.85 \%$ strain in Fig. $7($ a).

The 2D spatially resolved x-ray measurements in Fig. 4 (and in Larson et al., 2008) contain only rotational distortions and hence provide no information on $-e_{i k l} \partial_{k} \varepsilon_{l j}$, the elastic strain 
contribution to $\alpha_{i j}$ in Eq. (9). Accordingly, the dislocation density tensor measurements in Larson et al., (2008) correspond to the Nye tensor $\alpha_{i j}^{\mathrm{Nye}}$ defined in Eq. (10). Similarly, some dislocation density tensor determinations from electron backscattering diffraction patterns (e.g. Sun et al., 2000; Demir et al., 2009; Jiang et al., 2013) do not consider the elastic strains based on the insignificance of the elastic strain fields in comparison to the lattice rotation fields. However, a recent theoretical analysis (Acharya and Knops, 2014) suggested that the difference between Kröner and Nye tensors can be significant due to the possibility that the curl of an elastic strain field and a lattice rotation field might be comparable even when the two fields are different in magnitude. Considering the challenges facing current techniques for measuring the elastic strain and lattice rotation fields (Larson et al., 2008; Wilkinson and Randman, 2010; Wilkinson et al. 2014), the present work provides a necessary insight into the elastic strain field contribution to the dislocation density tensor.

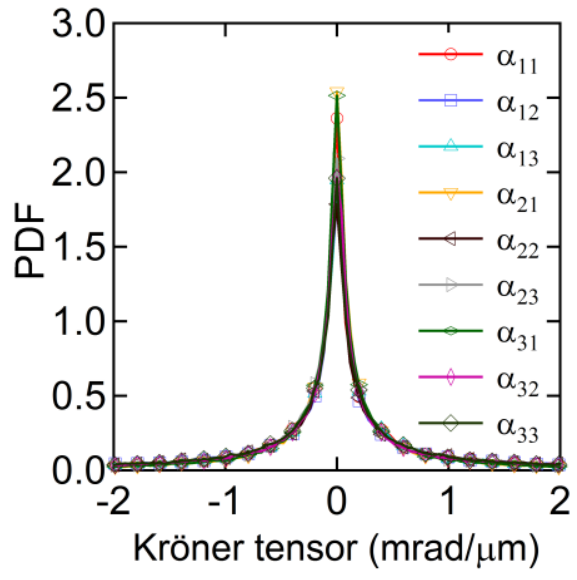

(a)

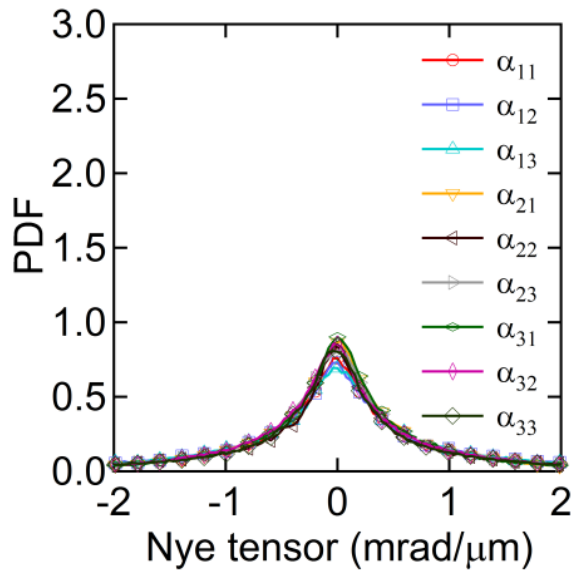

(b)

Fig.7. The PDF of the components of (a) Kröner dislocation density tensor and (b) Nye dislocation density tensor at strain level of $0.85 \%$.

The PDF of the Nye dislocation density tensor components are shown in Fig. 7(b). Comparison with Fig. 7(a) indicates that the elastic strain field contribution to the dislocation density tensor is 
significant. Evidently, the elastic strain contribution leads to the sharper peak in Fig. 7(a) along with a more narrow distribution width than in Fig. 7(b). At first glance, this observation might be thought of as an indication that the elastic strain contribution causes an overall decrease in the dislocation density tensor values. However, maps for dislocation density tensor norm shows that this inference is not necessarily true in general.

Fig. 8 shows a 2D map of the norm of the Nye dislocation density tensor; this it to be compared with the 2D map of the Kröner dislocation density tensor norm counterpart in Fig. 6(b) at a strain value of $0.85 \%$. The two figures confirm a significant effect of elastic strain in the dislocationfree regions. In such regions, excluding the elastic strain contribution yields non-zero values of the Nye tensor (shown as light blue color in Fig. 8) due to the non-vanishing gradient for lattice rotations. This observation is consistent with previous experimental measurements of both strain and curvature contributions for an elastically deformed silicon sheet (Larson et al., 2007, 2008); therein the elastic strain contribution was found to cancel the curvature contribution because $\mathrm{Si}$ at room temperature is brittle and elastic bending does not introduce dislocations. On the other hand, in dislocation rich zones, the elastic strain contribution acts differently, where it increases the dislocation density tensor norm in order to maintain a compact crystal. The elastic strain contribution is further displayed by plotting the PDF of the dislocation density norm, Fig. 9. The elastic strain contribution, again, pushes the distribution peak downward due to the cancelation of the curvature gradient with the elastic strain curl in dislocation free zones. It also weakens the distribution tail due to the accompanied increase in the dislocation density tensor norm in dislocation rich zones.

Turning to an analysis of the PDF, we have found that the dislocation density tensor norm distributions can be fit to lognormal distributions as shown in Fig. 9. Such a distribution is 
similar to that reported earlier (Hughes et al., 1997 and 1998; and Gurao and Suwas, 2014) regarding the distribution of misorientation angles, based on experimental measurements. The potential correspondence between the misorientation angle and the Nye dislocation density tensor is thus discussed in the following subsection.

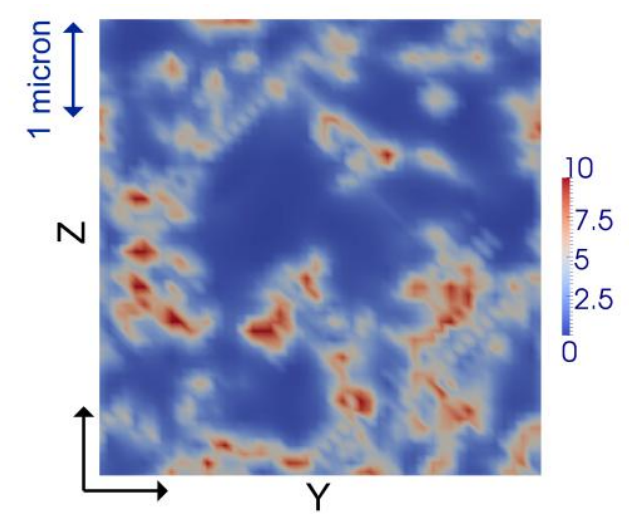

Fig. 8. A 2D map for the Nye dislocation density tensor norm (mrad/micron) calculated using Eq. (10) at strain level $0.85 \%$. This map is on the same plane as those in Figs. 6 (a) and (b).

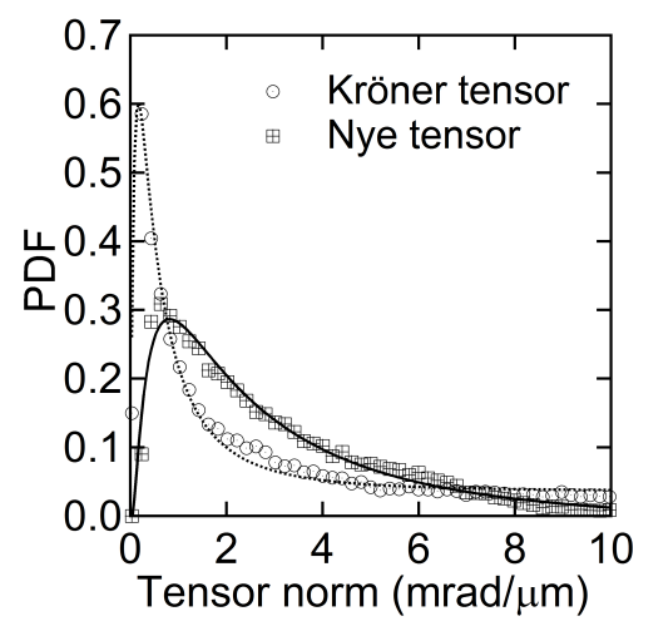

Fig. 9. PDF of the norms of Nye and Kröner dislocation density tensors at strain level of $0.85 \%$ strain. The PDFs are fitted into lognormal distribution.

In order to quantify the relative importance of the elastic strain and the lattice rotation contributions, $-e_{i k l} \partial_{k} \varepsilon_{l j}$ and $\partial_{j} \omega_{i}-\delta_{i j} \partial_{k} \omega_{k}$, to the dislocation density tensor, we plot in Fig. 
10(a) the ratio, $f_{E S}$, of the volume average norm of the elastic strain contribution to the volume average norm of the curvature contribution to the dislocation density tensor versus the applied tensile strain. We note that $f_{E S} \approx 0.85$ for the full range of strain levels considered; this implies that the volume averaged elastic strain contribution to the dislocation tensor is comparable (within 15\%) in magnitude to the curvature contribution for the simulations performed here. For dislocation-free, elastically-deformed regions the elastic strain and curvature contribution tensors components are known to be equal, but with opposite sign (Larson et al., 2007, 2008), meaning that the norms of both $-e_{i k l} \partial_{k} \varepsilon_{l j}$ and $\partial_{j} \omega_{i}-\delta_{i j} \partial_{k} \omega_{k}$ tensors are exactly equal. We note that $f_{E S} \approx 0.85$ at various strain levels implies further that the norms of the $-e_{i k l} \partial_{k} \varepsilon_{l j}$ and $\partial_{j} \omega_{i}-\delta_{i j} \partial_{k} \omega_{k}$ tensors are also comparable even in dislocation rich regions. In such regions, the $-e_{i k l} \partial_{k} \varepsilon_{l j}$ term increases the Kröner tensor norm in comparison to the Nye tensor norm, as can be noticed when comparing the 2D maps in Fig. 6(b) and Fig. 8.

We comment, however, that the fundamental significance of the elastic strain contribution to the dislocation density tensor is independent of its size relative to the rotation part. The Kröner dislocation density tensor in Eq. (9) provides an unambiguous measure of the "net" dislocation content within a given volume element through the elastic displacement incompatibility. That is, the incompatibilities that appear in the elastic distortion tensor $\left(\beta_{i j}^{e}\right.$ in Eq. (6)) are the algebraic sum of the elastic strain and lattice rotation tensors (as indicated in Eq. (1)). Accordingly, the Kröner tensor vanishes for dislocation-free volume elements where the elastic displacement field is compatible (i.e. $\beta_{i j}^{e}$ is curl-free), and provides a measure of the dislocation-induced incompatibility in dislocation-rich volume elements. On the other hand, given by the curvature 
part of the Kröner tensor, the Nye tensor provides only an estimate of a part of the incompatibility due to dislocations in dislocation-rich volume elements. Nye tensor can also have non vanishing components due to lattice rotation variations in dislocation-free volume elements. As is well known, the Nye tensor provides an unambiguous measure of the net dislocation content only in the limit of vanishing elastic strains.

The above analysis shows that ignoring the elastic strain field in the determination of the dislocation density tensor leads, on average, to some error that is manifested as a nonzero value for $\alpha_{i j}$ in dislocation free zones and, at the same time, to an underestimation of $\alpha_{i j}$ in dislocation rich zones. Since it is usual to use point-wise measurements to determine average GND densities, we now proceed to investigate how volume average values would be affected if the elastic strain fields were neglected in our simulation for uniaxial tensile deformation of $\mathrm{Cu}$. Volume average Kröner and Nye dislocation density tensor norms, $\langle\|\boldsymbol{\alpha}\|\rangle$, are displayed in Fig. 10(b) for tensile strains ranging from 0.15 to $0.85 \%$. The figure shows that at low strain (i.e., low dislocation density) the Nye and Kröner tensor norms are roughly equal. However, at higher strain levels (i.e., higher dislocation densities), the Kröner tensor norm is about $12 \%$ higher. Componentwise, this implies only that the tensor components are larger in absolute value. The fact that the average norms of the Kröner and Nye tensors have quite similar values within the strain range under study is not in contradiction with the fact that the strain and rotation contributions to the dislocation density tensor are comparable in magnitude, as depicted in Fig. 10(a). Recalling that the Kröner tensor norm is larger than the Nye tensor norm in dislocation-rich zones but smaller in dislocation-free zones, the corresponding norm averages will be affected by the dislocation density in the crystal. For low dislocation densities, the volume averaged Kröner tensor norm will be dominated by the zero values in volume elements not containing dislocations, whereas 
the nonzero values for the Nye tensor norm in such volume elements could make the Nye tensor average larger than the average Kröner tensor norm.

As the dislocation density increases, however, the larger values of the Kröner tensor norm in dislocation-rich volume elements can be expected to dominate, resulting in an increase in the Kröner tensor norm relative to the Nye tensor norm, as observed in figure 10(b). This trend will have significance in connection with experimental investigations of the evolution of volume average GND densities as a function of increasing strain (i.e. different dislocation density values). That is, the error incurred in the determination of average GND densities will increase with increasing strain level (i.e., dislocation density) as figure 10(b) indicates. Accordingly, it will be important to extend such comparisons to larger applied strains, because the magnitude of the errors will have to be weighed against the fact that the time and effort required for current elastic strain determinations using 3DXM is significant with presently available $\mathrm{x}$-ray facilities and the absence of reference strains in deformed samples is challenging for present EBSD techniques (Wilkinson et al., 2014).

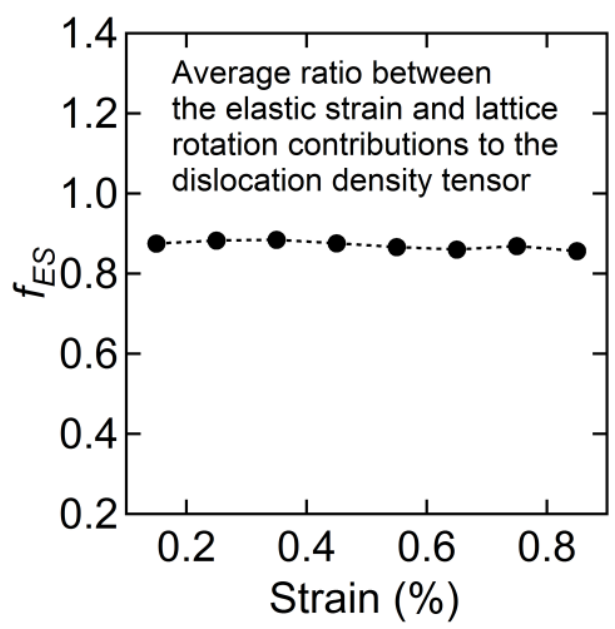

(a)

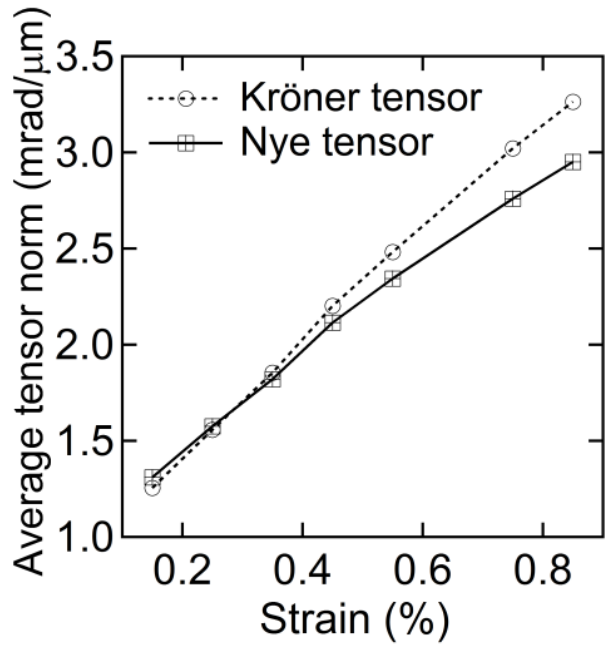

(b) 
Fig. 10. (a) The ratio between the elastic strain and the curvature contributions to the dislocation density tensor $\left(f_{E S}\right)$ versus the crystal strain level. (b) The evolution of the average norm of Kröner and Nye dislocation density tensors with crystal strain level.

The radial correlation function for the dislocation density tensor norm is shown in Fig. 11. The figure reveals a faster decay for the correlation function including the elastic strain contribution, which is consistent with the above discussed observation of shorter correlation lengths for the elastic strain than for the lattice rotation fields. This effect is reflected in the calculated correlation length values $\ell_{0}$ for the dislocation density tensor norm, which was found to decrease from 0.25 to 0.22 micrometers when adding the elastic strain contribution (at strain level of $0.85 \%$ ). Recall from section 5.2 that, at a strain level of $0.85 \%$, the correlation lengths for the elastic strain and the lattice rotation components were in the range 0.21-0.24 micrometers and 0.40-0.57 micrometers, respectively. We note therefore that the elastic strain PCF corresponds to a different representation of the spatial features of the underlying dislocation system than the lattice rotation PCF.

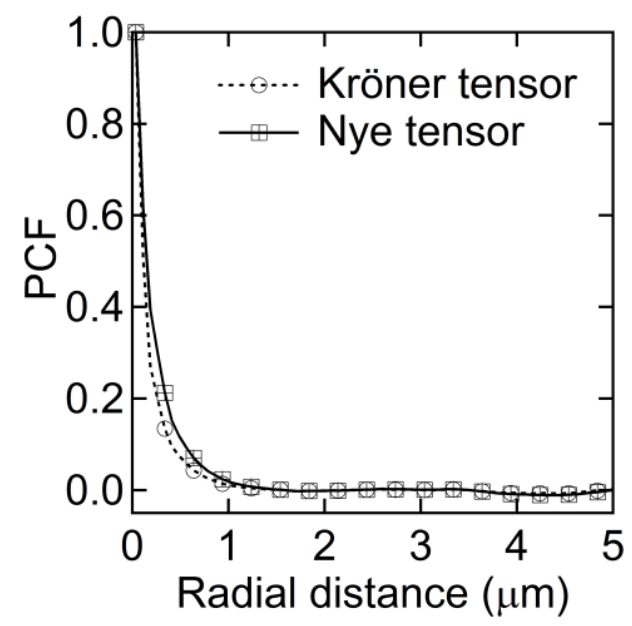

Fig. 11. Radial self-correlation of the norm of the Nye and Kröner's dislocation density tensors at a crystal strain level of $0.85 \%$. 


\subsection{The misorientation angle versus Nye dislocation density tensor}

A correspondence between the misorientation angle (differential rotation over small distances) and the Nye dislocation density tensor is now explored. This is motivated by the similarity between the PDFs for the Nye dislocation density norm in Fig. 9 and the misorientation angles across cell boundaries reported in the literature (Hughes et al., 1997 and 1998; Gurao and Suwas, 2014). Although the difference in the strain levels between the current simulations $(<1.0 \%)$ and to those discussed in the literature $(>100 \%)$ is very large, the character of the misorientation PDF and the scaling of its average value were found to persist over many length scales and for various deformation levels and crystal structures (Gurao and Suwas, 2014). Therefore, it is of interest to investigate the statistics of the misorientation angles in our simulations to test the scaling behavior for very small strain levels.

The misorientation angle between two neighboring crystal points $p^{1}$ and $p^{2}$ is given by $\left\|\boldsymbol{\omega}^{2}-\boldsymbol{\omega}^{1}\right\|$, where $\boldsymbol{\omega}^{i}$ is the rotation axial vector at point $p^{i}$. For a point $p^{i}$, it is thus possible to calculate three misorientation angles between the point and each neighboring points in $x, y$, and $z$-directions. In previous analyses, some authors (Hughes et al., 1997 and 1998) statistically analyzed the misorientation angle measurements separately across cell boundaries formed by statistically stored and geometrically necessary boundaries, whereas others (Gurao and Suwas, 2014) combined the measurements across both boundary types. We note that in the current analyses, subjective sub-division of the dislocation system microstructure of this nature does not arise. Fig. 12(a) shows the PDF for misorientation angles side by side with the corresponding 
distributions for the Nye dislocation density tensor norm in Fig. 12(b) for 3 different strain levels. It is observed that both the misorientation angle PDF and the Nye tensor norm PDFs can be fit to lognormal distributions. The lognormal character of the distributions implies a multiplicative effect for the misorientation angles. This is different from the components of the elastic strain and lattice rotation fields, which have normal (Gaussian) distributions (Fig. 1(a) and Fig. 2 (a) and (b)) due to the additive nature of these fields; i.e. the field calculated at any point is the summation of the fields generated by all dislocations. The (axial) misoreintation angles are, however, calculated as the vector difference norm, $\left\|\boldsymbol{\omega}^{j}-\boldsymbol{\omega}^{i}\right\|$, where the multiplication included in the norm calculation process is presumably the source of the multiplicative effect. This is also observed for the Nye dislocation density tensor norm PDF (Fig. 12(b)), which also reveals a lognormal distribution, although the individual tensor components were normally distributed (Fig. 7(b)). Fig. 12 shows a similarity between the misorientation angles and the Nye tensor norm distributions, and also their dependence on the strain level, which underscores the direct correspondence between misorientation angles and the Nye dislocation density tensor.

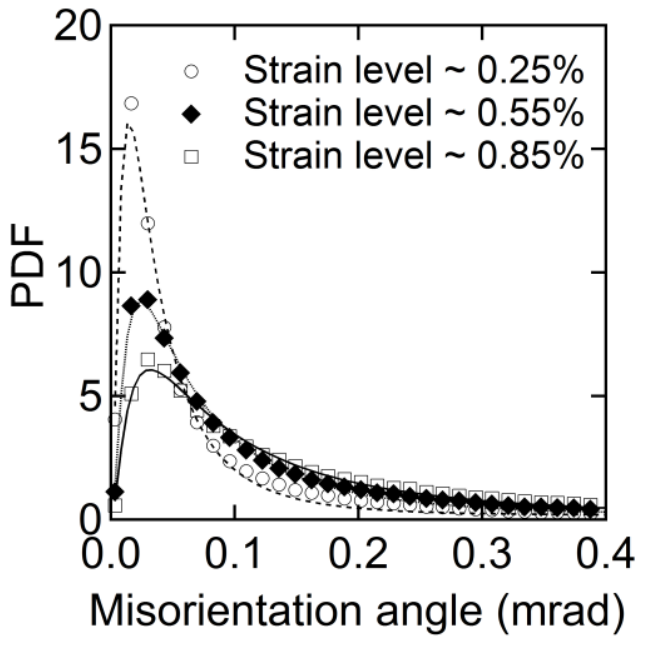

(a)

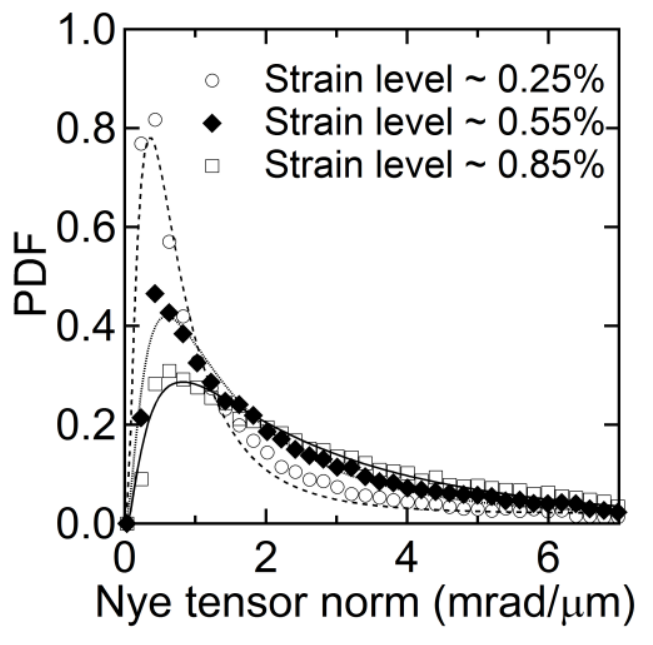

(b) 
Fig. 12. The PDF for (a) the misorientation angle at various crystal strain levels. (b) Nye dislocation density tensor norm. The PDFs are fitted with lognormal distribution in both figures.

The average misorientation angles are plotted versus strain in Fig. 13(a).The figure indicates a square root power law relationship between the average misorientation angle and the strain level, $\langle\theta\rangle \propto \sqrt{\varepsilon}$, which is similar to that reported in literature (Hughes et al., 1997 and 1998; Gurao and Suwas, 2014). This emphasizes further the generality of misorientation angle scaling across length scales. Fig. 13(a) also shows the evolution of the volume average of the Nye dislocation density tensor norm with strain level, which shows a power law scaling, $\left\langle\left\|\boldsymbol{\alpha}^{N y e}\right\|\right\rangle \propto \sqrt{\varepsilon}$. These results suggest that, for the case of monotonic loading and within the range of strains considered here, there is a linear relationship between average Nye dislocation density tensor norms and average misorientation angles, as shown in Fig. 13(b). Of course, additional analysis over a wider strain range and different loading conditions is needed in order to reveal the precise nature of the relationship. Nevertheless, the importance of exploring such relationships resides in its potential to link the rich transmission electron microscopy (TEM), orientation imaging microscopy (OIM) and electron backscattering diffraction (EBSD) literature on misorientation angle analyses, recent spatially resolved 3D X-ray lattice rotation measurements, and plasticity theories that adopt the dislocation density tensor as their constitutive variable. 


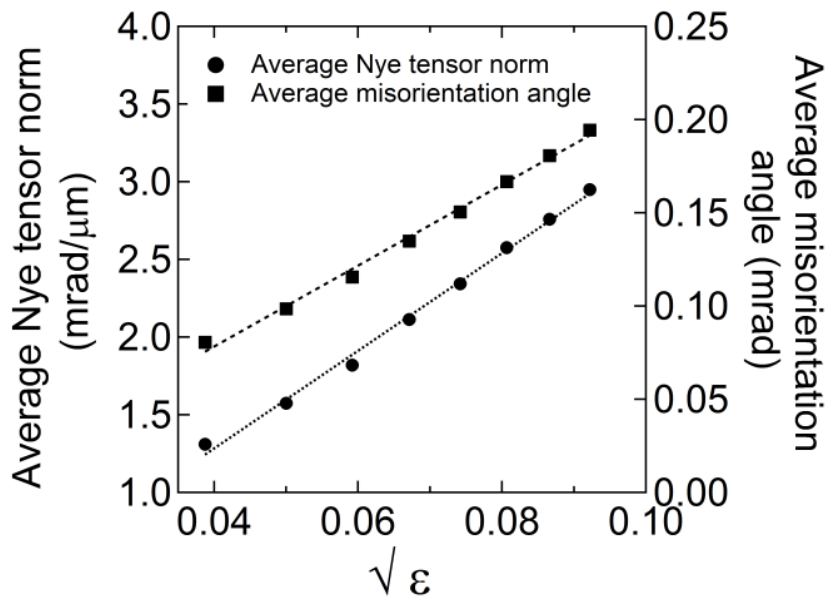

(a)

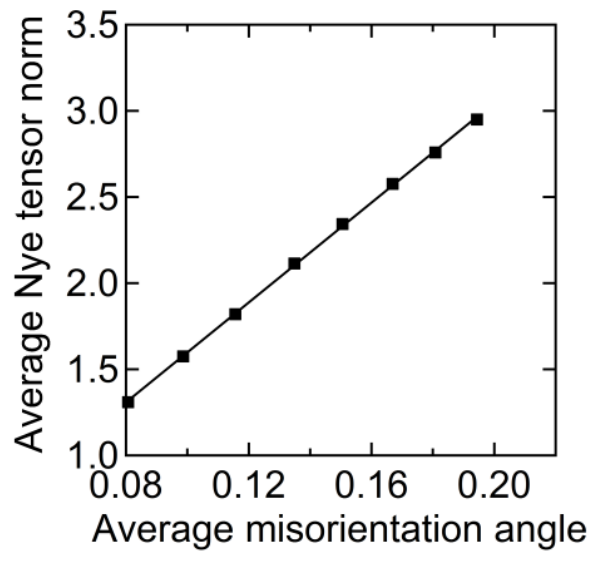

(b)

Fig.13. (a) The evolution of the average misorientation angle and Nye dislocation density tensor norm during the deformation process. The lines represent a linear fitting of the data points. (b) The average misorientation angle versus the average Nye dislocation density tensor during the deformation process. The line also represents a linear fitting of the data points.

\section{Concluding remarks}

A statistical analysis of the elastic distortion fields of dislocations in deforming crystals has been performed. This analysis was motivated by the recent advances in 3DXM enabling the nondestructive measurement of the local elastic strain and lattice rotation fields in 3D with submicron spatial resolution. Analysis of the simulated elastic strain fields has revealed statistical features similar in general to those previously reported for the internal stress field regarding the shapes of the PDFs and the character of the correlations (Deng, 2008). The analysis has shown that the lattice rotation field has longer correlation lengths than its elastic strain counterpart, which is attributed to the more frequent sign alteration, on average, around dislocation lines for the elastic strain in comparison with the lattice rotation field. Determinations of the correlation lengths for both the elastic strain and rotation fields and for the dislocation density tensor norm have shown that the correlation length for the dislocation density tensor 
norm follows that of the elastic strain field more closely, both of which were found to have correlation lengths approximately half that for the rotation field.

The elastic strain field for tensile strained $\mathrm{Cu}$ was found to make a non-negligible contribution to the dislocation density tensor, indicating that the difference between the Nye tensor and the Kröner tensor is of significance. Although experimental measurements of the elastic strain contribution to the dislocation density tensor present significantly larger challenges than the rotational part and are often not performed, the analysis provided here illustrates that the Kröner tensor provides a more complete measure of the underlying dislocation structure.

Statistical analysis revealed the PDF of the misorientation angles to follow a lognormal distribution, in addition to a (square root) power law scaling of the average value of the misorientation angles with strain. The close correlation between our analyses and previous experimental observations at much larger strain levels emphasizes the generality of the average misorientation angle scaling. The analysis over the restricted strain range considered here demonstrates a direct, linear relationship between the average misorientation angle and the average Nye dislocation density tensor norm, as expected.

The PDFs of the measured and simulated lattice rotation fields were found to be similar in terms of their full width at half maxima and their evolution with the deformation level. However, the dislocation dynamics simulations of a $5 \mu \mathrm{m}$ cube could not be expected to reproduce the long range (10s of micrometers) rotation zones that were observed experimentally. This result underscores the need to perform simulations using larger domains for better comparison with experiments. We remark finally that the statistical analysis presented provides the basis for a theoretical foundation for testing the fidelity of dislocation dynamics models through direct 
comparisons between the computationally and experimentally determined elastic distortion fields in deformed crystals. It must be born in mind though that confronting discrete dislocation dynamics simulation models with experiments will be limited to the cases of infinitesimal deformation until newer models capable of handling finite lattice rotation have been implemented.

\section{Acknowledgments}

Research was supported in part by the U.S. Department of Energy, Basic Energy Sciences, Materials Sciences and Engineering Division under contract \# DE-FG02-08ER46494 at Florida State University (MSM). Research at ORNL was supported by the U.S. Department of Energy, Basic Energy Sciences, Materials Sciences and Engineering Division (BCL). Use of the 34-ID beamline at the Advanced Photon Source was supported by the U.S. Department of Energy, Office of Basic Energy Sciences, Scientific User Facilities Division operated for the DOE Office of Science by Argonne National Laboratory under Contract No. DE-AC02-06CH11357 (JZT).

\section{References}

Acharya, A., 2001. A model of crystal plasticity based on the theory of continuously distributed dislocations. Journal of the Mechanics and Physics of Solids 49, 761-784.

Acharya, A., Knops, R.J., 2014. An observation on the experimental measurement of dislocation density. Journal of Elasticity 114, 275-279.

Arsenlis, A., Cai, W., Tang, M., Rhee, M., Oppelstrup, T., Hommes, G., Pierce, T. G., Bulatov, V. V., 2007. Enabling strain hardening simulations with dislocation dynamics. Modeling and Simulation in Materials Science and Engineering 15, 553-595. 
Britton, T.B., Jiang, J., Clough, R., Tarleton, E., Kirkland, A.I., Wilkinson, A.J., 2013. Assessing the precision of strain measurements using electron backscatter diffraction - Part 2: Experimental demonstration. Ultramicroscopy 135, 136-141.

Cai, W., Arsenlis, A., Weinberger, C.R., Bulatov, V.V., 2006. A non-singular continuum theory of dislocations. Journal of the Mechanics and Physics of Solids, 54, 561-587.

Cleveringa, H.H.M., Van der Giessen, E., and Needleman, A., 1999. A Discrete Dislocation Analysis of Bending. International Journal of Plasticity, 15, 837-868.

Demir, E., Raabe, D., Zaafarani, N., Zaefferer, S., 2009. Investigation of the indentation size effect through the measurement of the geometrically necessary dislocations beneath small indents of different depths using EBSD tomography. Acta Materialia 57, 559-569.

Deng, J., El-Azab, J., 2007. Dislocation pair correlation from dislocation dynamics simulations. Journal of Computer-Aided Materials Design 14, 295-307.

Deng, J., El-Azab, A., Larson, B.C., 2008. On the elastic boundary value problem of dislocations in bounded crystals. Philosophical Magazine 88, 3527-3548.

Deng, J., 2008. Statistical characterization of dislocation ensembles. Ph.D. dissertation, Florida State University.

Devincre, B., Madec, R., Monnet, G., Queyreau, S., Gatti, R., \& Kubin, L. (2011). Modeling crystal plasticity with dislocation dynamics simulations: The 'microMegas' code. Mechanics of Nano-objects. Presses de l'Ecole des Mines de Paris, Paris, 81-100.

El-Dasher, B.S., Adams, B.L., Rollett, A.D. (2003). Viewpoint: experimental recovery of geometrically necessary dislocation density in polycrystals. Scripta Materialia, 48, 141-145.

Gao, H., Huang, Y., Nix, W., Hutchinson, J., 1999. Mechanism-based strain gradient plasticity. I. $38 \mid \mathrm{P}$ a g e 
Theory. Journal of the Mechanics and Physics of Solids 47, 1239-1263.

Gao, H., Huang, Y., 2003. Geometrically necessary dislocation and size-dependent plasticity. Scripta Materialia 48, 113-118.

Ghoniem, N. M., Tong, S. H., Sun, L. Z., 2000. Parametric dislocation dynamics: A thermodynamics-based approach to investigations of mesoscopic plastic deformation. Physical Review B (Condensed Matter) 61, 913-927.

Gurao, N. P., Suwas, S., 2014. Generalized scaling of misorientation angle distributions at meso-scale in deformed materials. Scientific Reports 4, 5641.

Hirth, J.P., Lothe, J., 1982. Theory of Dislocations. John Wiley and Sons, New York.

Huang, Y., Gao, H., Nix,W., Hutchinson, J., 2000. Mechanism-based strain gradient plasticity. II. Analysis. Journal of the Mechanics and Physics of Solids 48, 99-128.

Hughes, D.A., Chrzan, D.C., Liu, Q. \& Hansen, N, 1998. Scaling of Misorientation Angle Distributions. Phys. Rev. Letts. 81, 4664-4667 .

Hughes, D.A., Liu, Q., Chrzan, D.C. \& Hansen, N, 1997. Scaling of microstructural parameters: Misorientations of deformation induced boundaries. Acta Mater. 45, 105-112 .

Jiang, J., Britton, T.B.,Wilkinson, A.J., 2013. Evolution of dislocation density distributions in copper during tensile deformation. Acta Materialia, 61 (19), 7227-7239.

Kacher, J., Landon, C., Adams, B.L., Fullwood, D., 2009. Bragg's law diffraction simulations for electron backscatter diffraction analysis. Ultramicroscopy, 109, 1148-1156.

Kröner, E., 1981. Continuum theory of defects. in Physics of Defects, Edited by Balian R, Kleman M and Poirier J (North-Holland, Amsterdam), 217-315. 
Larson, B. C., Yang, W., Ice, G. E., Budia, J., Tischler, J. Z., 2002. Three-dimensional X-ray structural microscopy with submicrometre resolution. Nature 415, 887-890.

Larson, B. C., El-Azab, A., Yang, W., Tischler, J. Z., Liu, W., Ice, G. E., 2007. Experimental characterization of the mesoscale dislocation density tensor. Philosophical Magazine 87, 13271347.

Larson, B. C., Tischler, J. Z., El-Azab, A., Liu, W., 2008. Dislocation Density Tensor Characterization of Deformation Using 3D X-Ray Microscopy. Journal of Engineering Materials and Technology 130, (021024) 1-10.

Larson, B.C., Levine, L.E., 2013 Submicrometre-resolution polychromatic three-dimensional Xray microscopy. Journal of Applied Crystallography 46, 153-164.

Levine, L.E., Larson, B.C., Yang, W., Kassner, M.E., Tischler, J.Z., Delos-Reyes, M.A., Fields, R.J., Liu, W., 2006, X-ray microbeam measurements of individual dislocation cell elastic strains in deformed single-crystal copper, Nature Materials 5, Issue 8, 619-622.

Limkumnerd, S., Sethna, J., 2007. Stress-free states of continuum dislocation fields: Rotations, grain boundaries, and the Nye dislocation density tensor. Physical Review B 75, (224121) 1-9.

Nye, J. F., 1953. Some geometrical relations in dislocated crystals. Acta Metallurgica 1, 153162.

Sun, S., Adams, B. L., King, W. E., 2000. Observations of lattice curvature near the interface of a deformed aluminum bicrystal. Philosophical Magazine A: Physics of Condensed Matter, Structure, Defects and Mechanical Properties 80, 9-25.

Villert, S., Maurice, C., Wyon, C., Fortunier, R., 2009. Accuracy assessment of elastic strain measurement by EBSD. Journal of Microscopy 233, 290-301. 
Wilkinson, A.J., Meaden, G., Dingley, D.J., 2006. High-resolution elastic strain measurement from electron backscatter diffraction patterns: New levels of sensitivity. Ultramicroscopy 106, 307-313.

Wilkinson, A.J., Randman, D., 2010. Determination of elastic strain fields and geometrically necessary dislocation distributions using electron back scatter diffraction. Philosophical Magazine 90, 9, 1159-1177.

Wilkinson, A.J., Britton, T.B., Jiang. J., Karamched, P.S., 2014. A review of advances and challenges in EBSD strain mapping. IOP Conf. Series: Materials Science and Engineering 55, 012020.

Yang, W., Larson, B.C., Ice, G.E., Tischler, J.Z., Budai, J.D., Chung, K.-S., Lowe, W.P., 2003. Spatially resolved poisson strain and anticlastic curvature measurements in $\mathrm{Si}$ under large deflection bending. Applied Physics Letters, 82, 22 (2), 3856-3858.

Zaafarani, N., Raabe, D. , Roters, F., Zaefferer, S., (2008). On the origin of deformation-induced rotation patterns below nanoindents. Acta Materialia, 56 (1), 31-42.

Zaefferer S., Wright S.I., Raabe D., (2008). Three-dimensional orientation microscopy in a focused ion beam-scanning electron microscope: A new dimension of microstructure characterization. Metallurgical and Materials Transactions A: Physical Metallurgy and Materials Science, 39 (2), 374-389. 


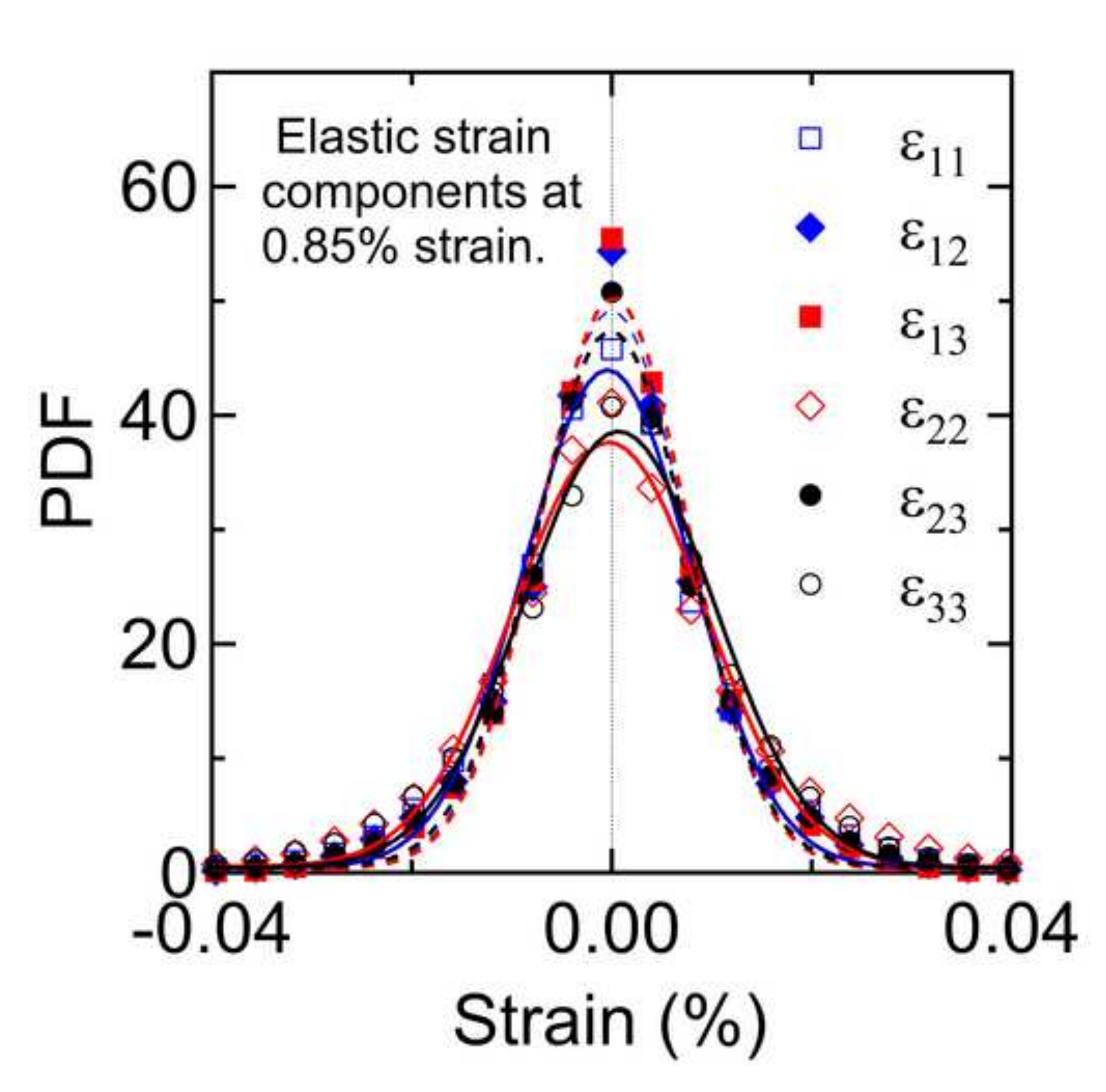

Figure_01a_color

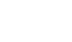

(2n
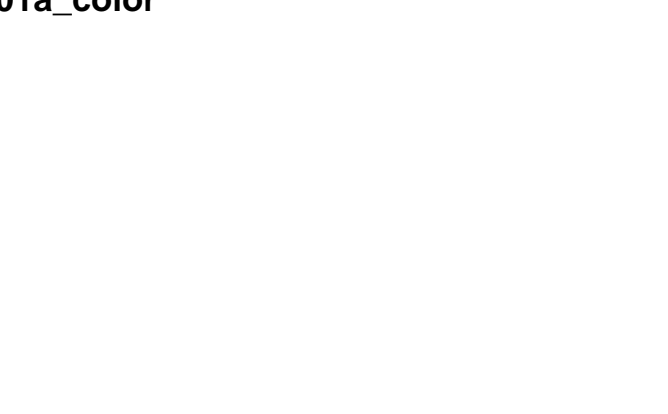

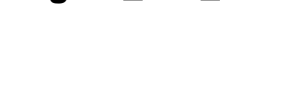

. 


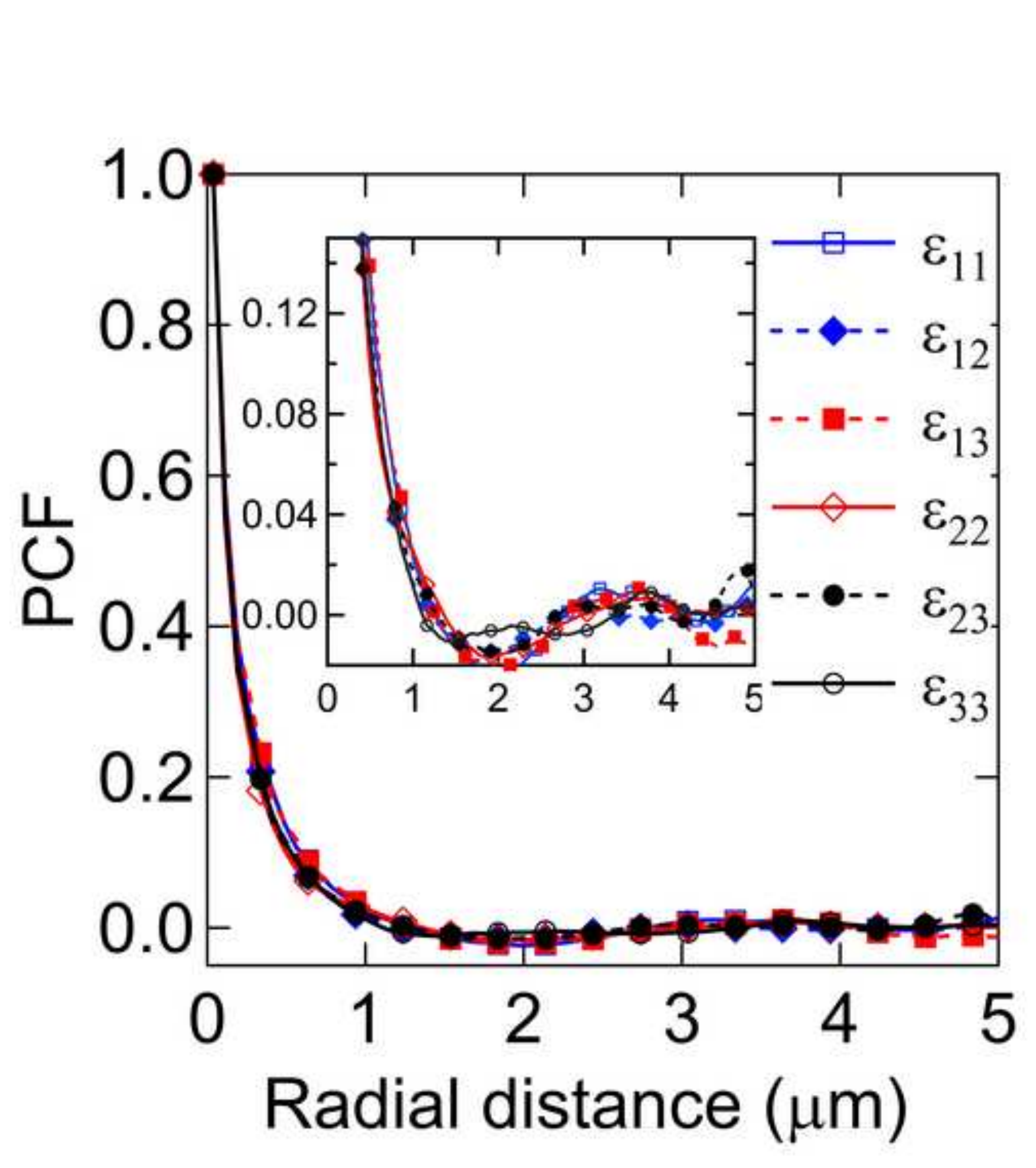

Figure_01b_color

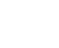
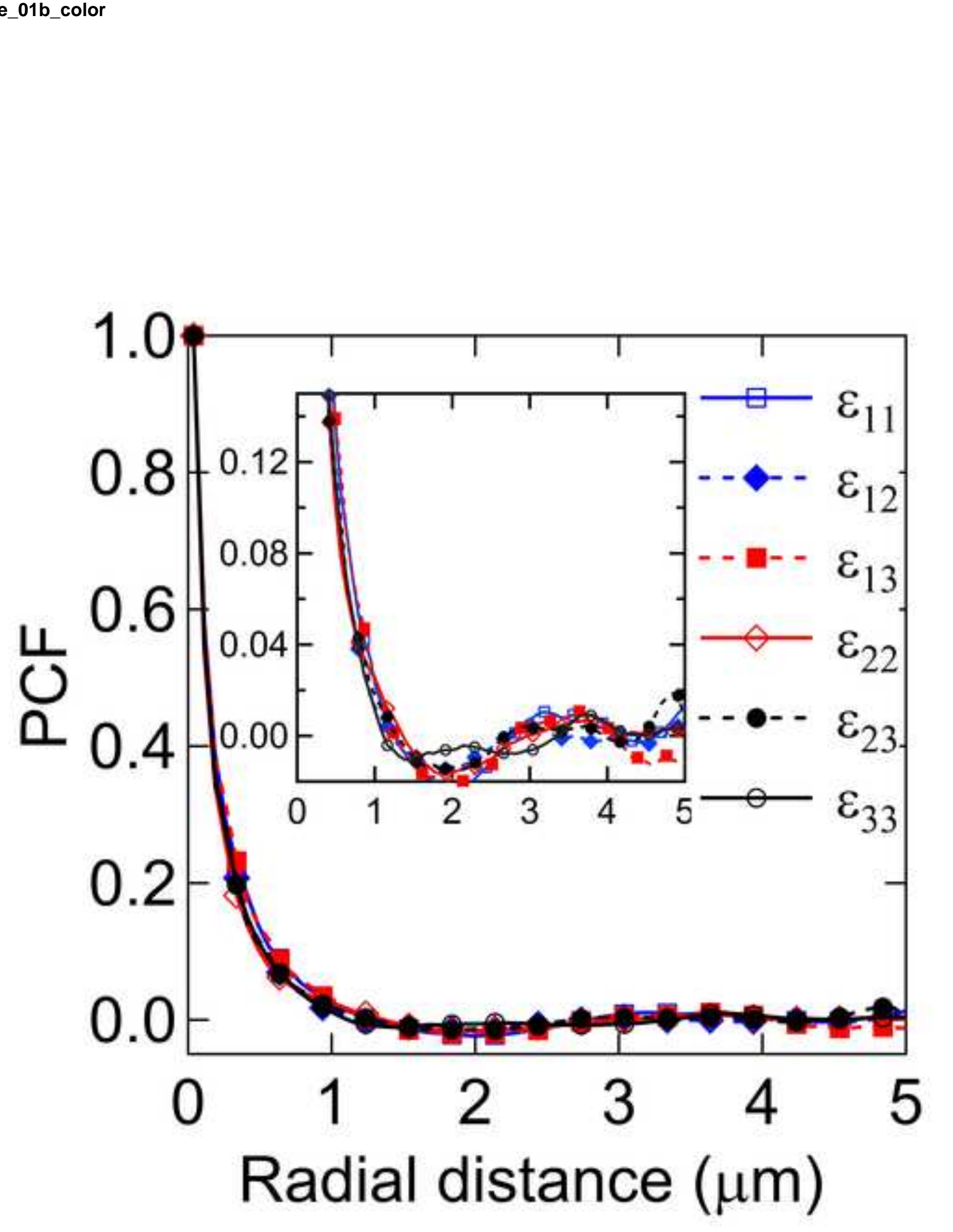


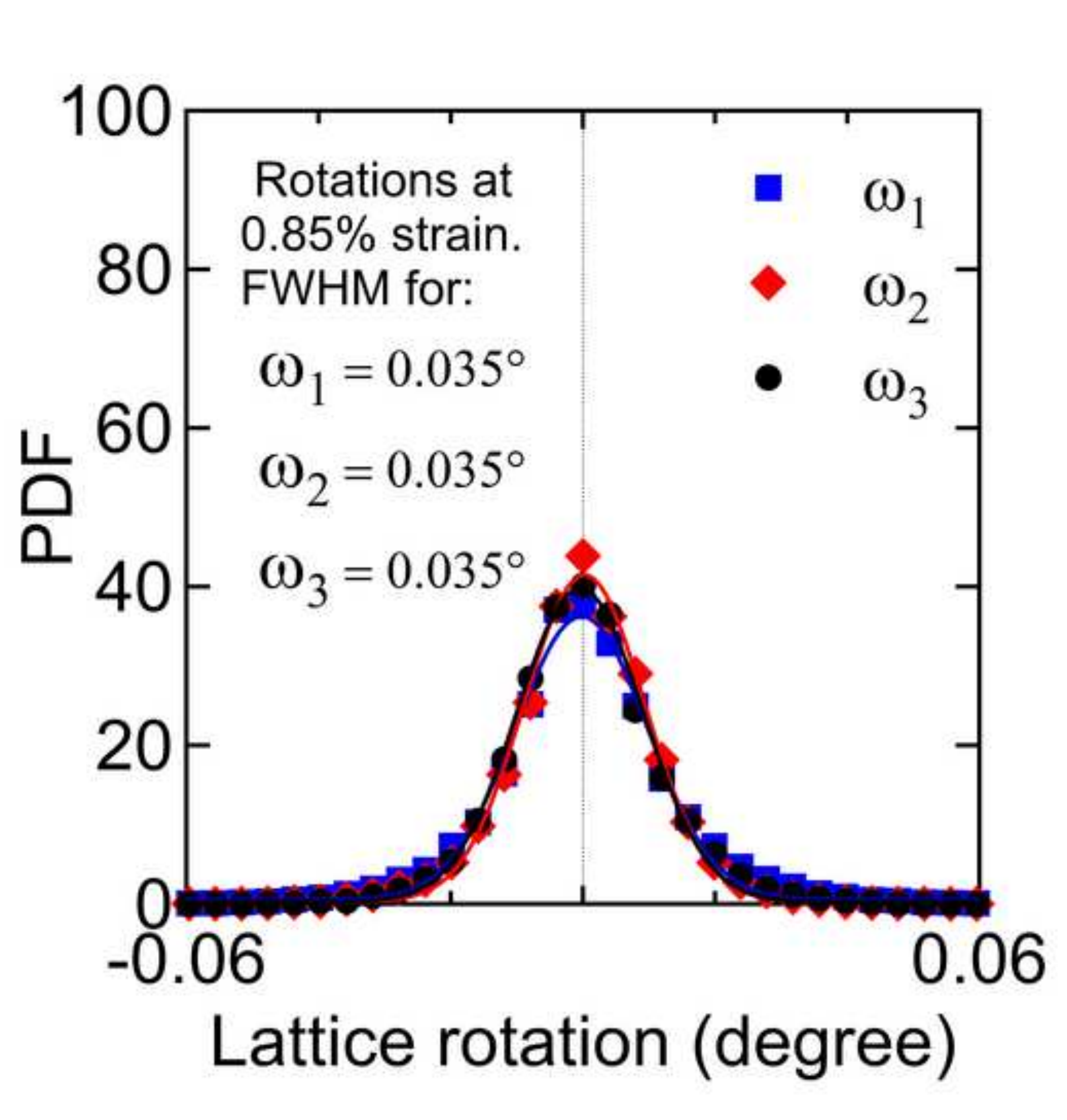

Figure_02b_color

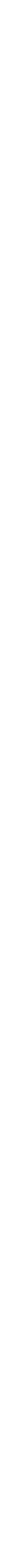

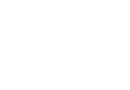
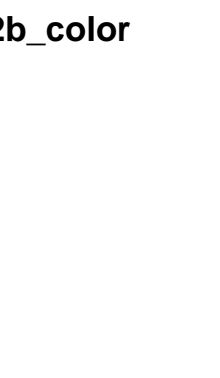

\footnotetext{
(atc)
}
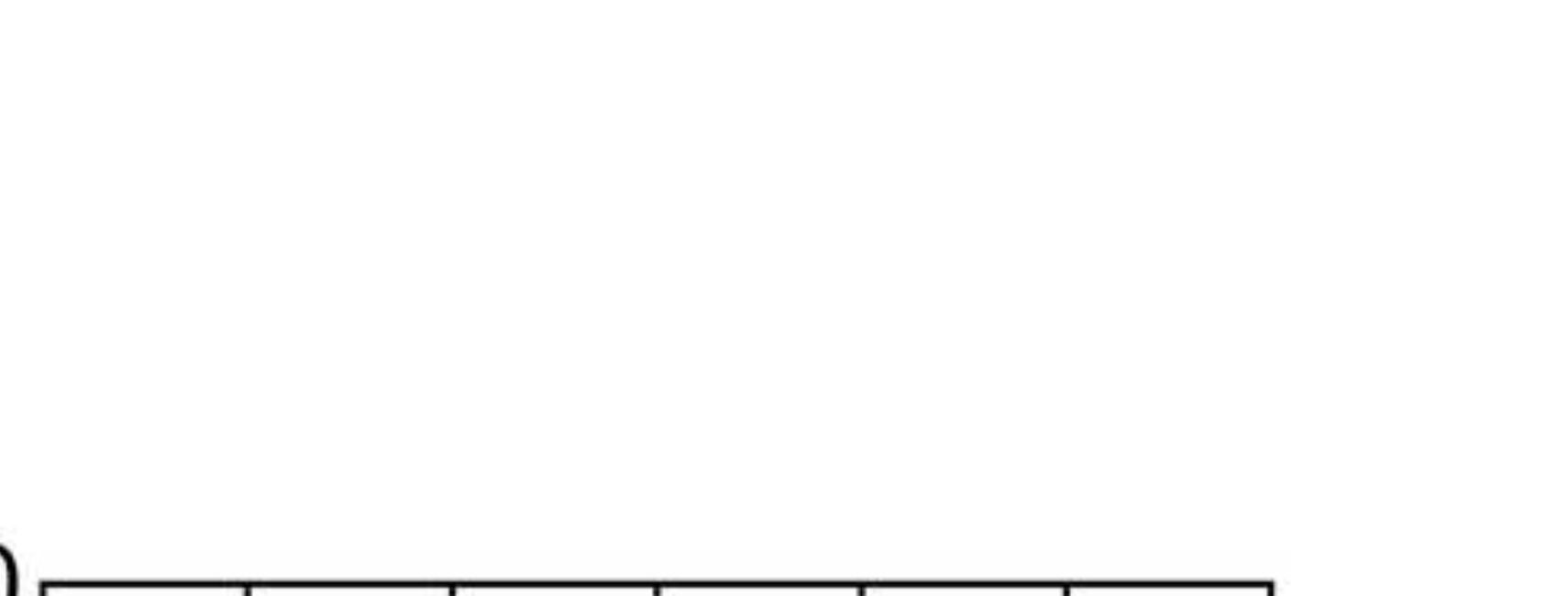

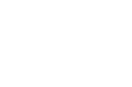
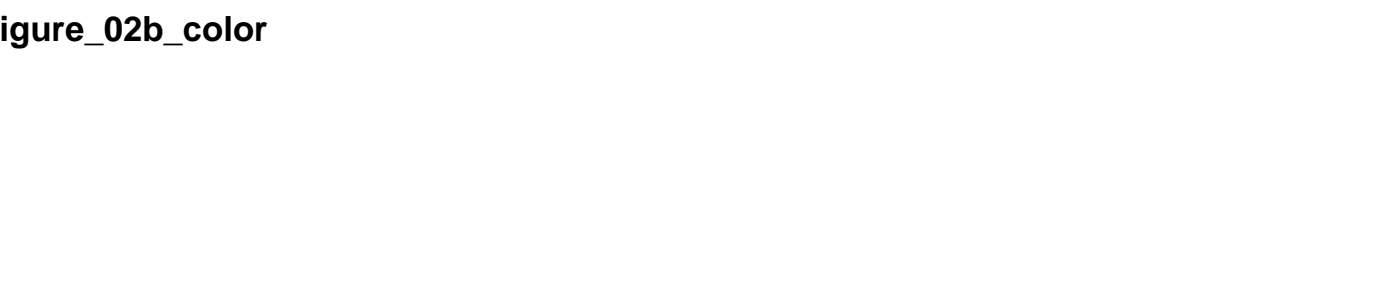

Fing
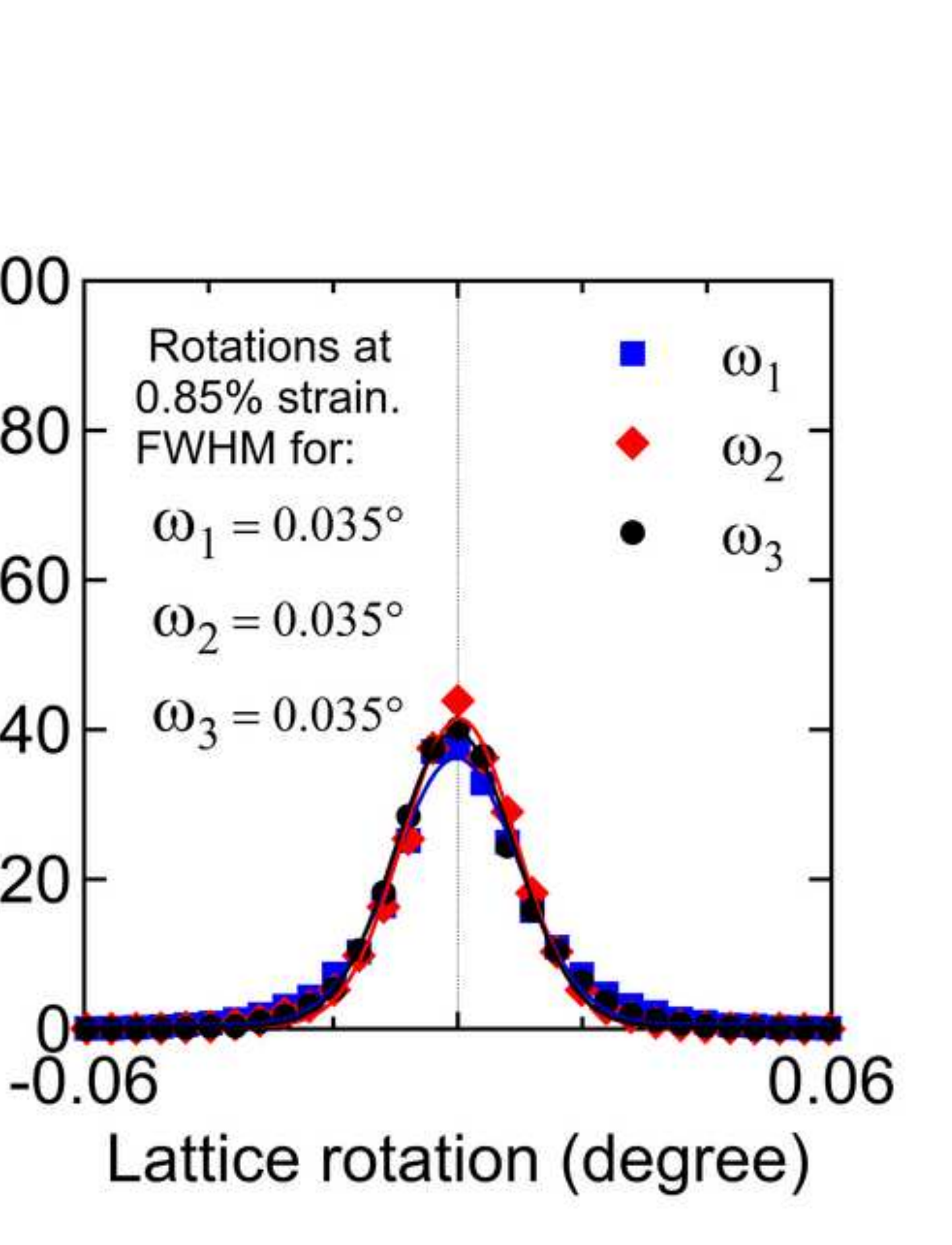

\section{Lattice rotation (degree)}

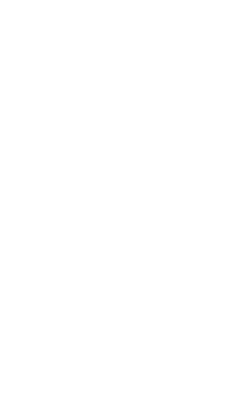




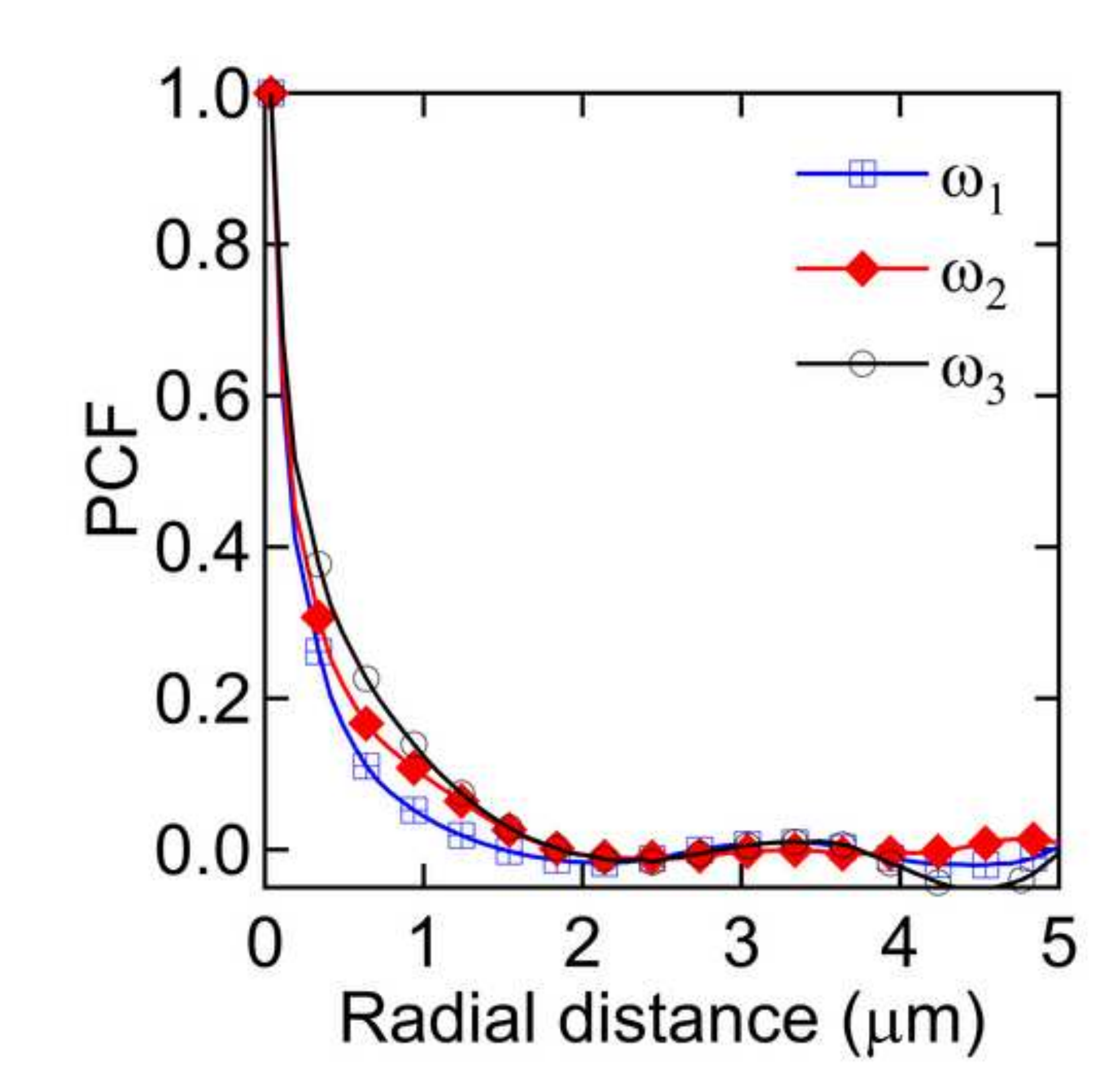

Figure_02c_color

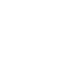




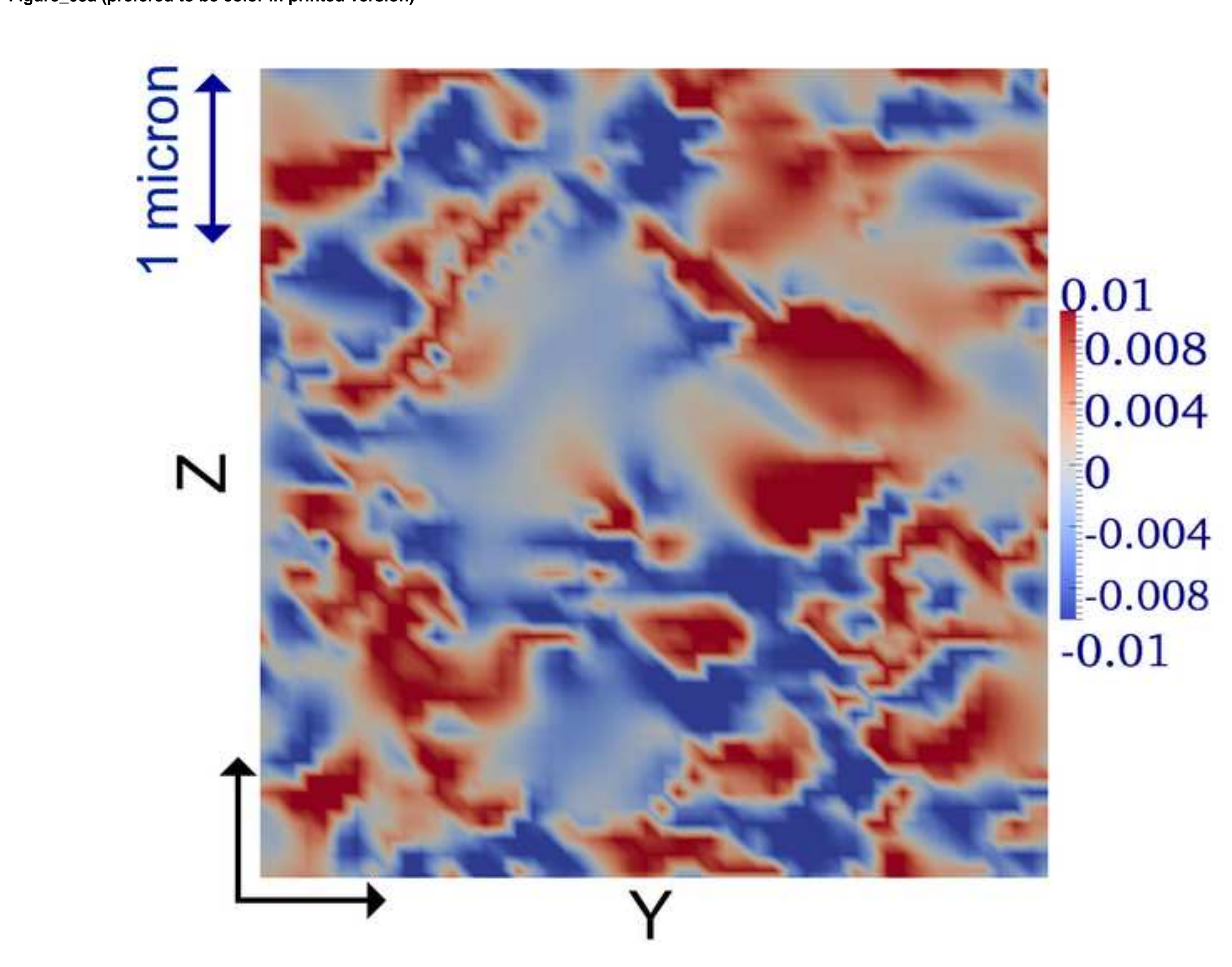

\section{.01}

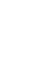

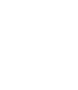



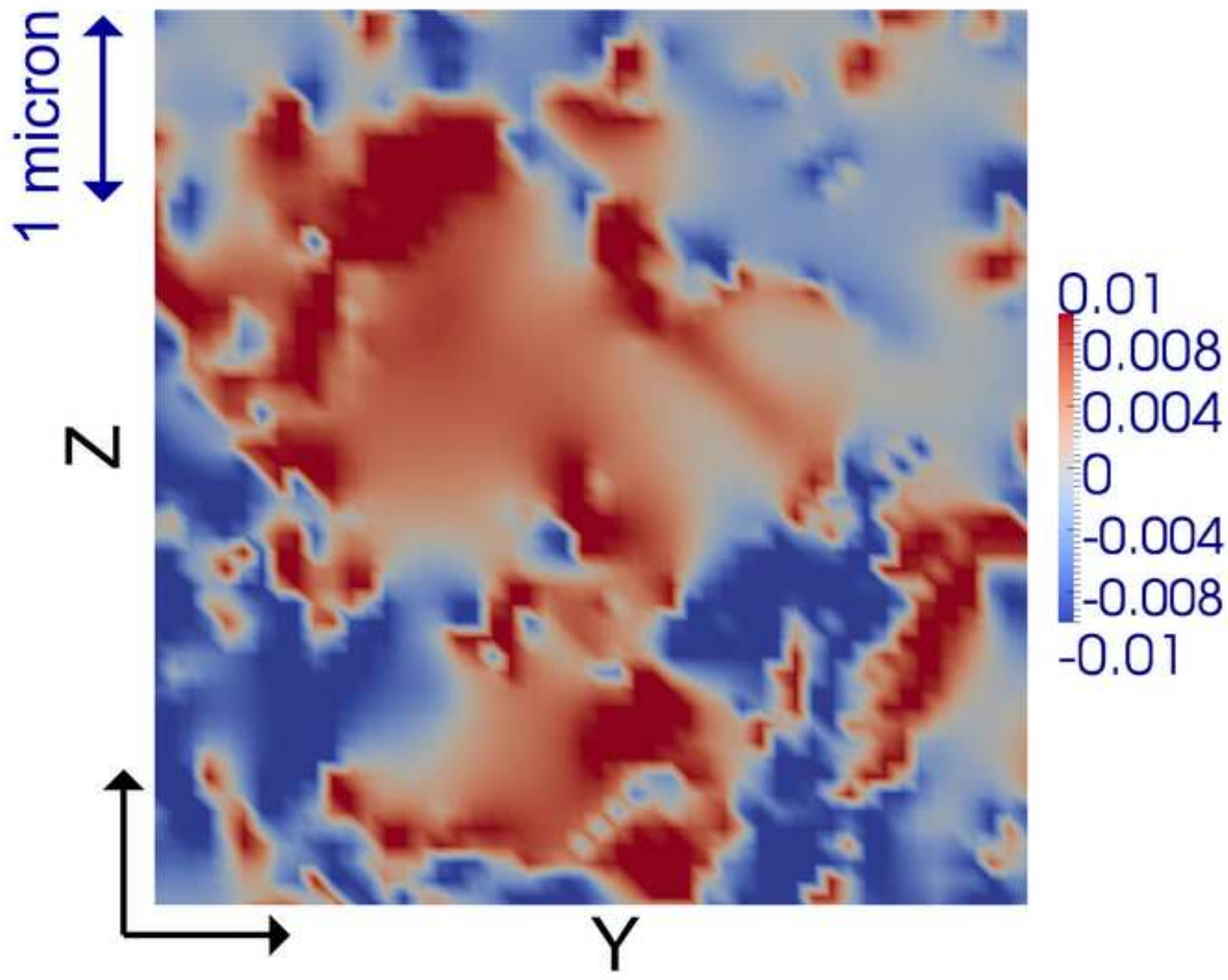
0.004 0 $-0.004$ $-0.008$ $-0.01$ 
Figure_04 (prefered to be color in printed version)

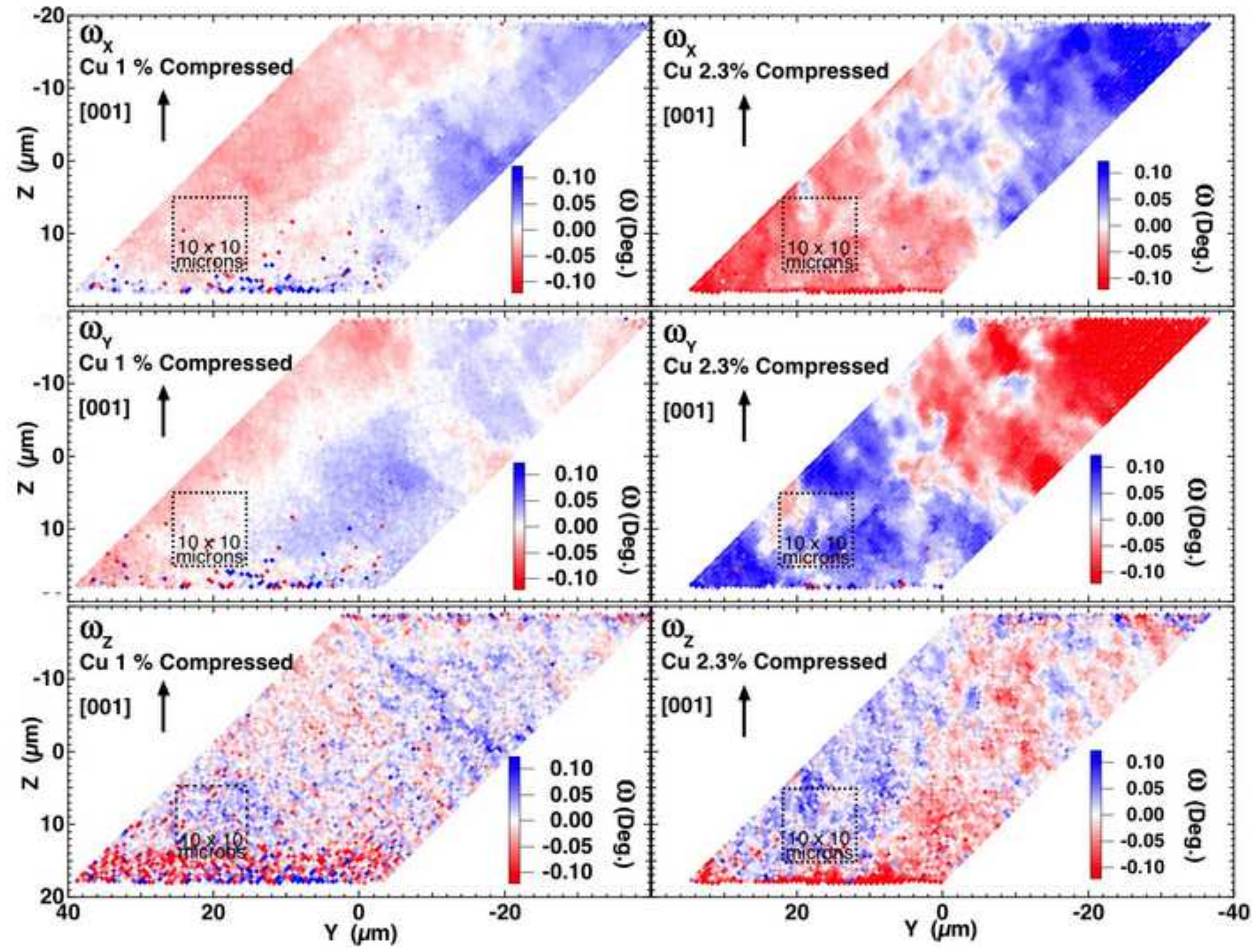




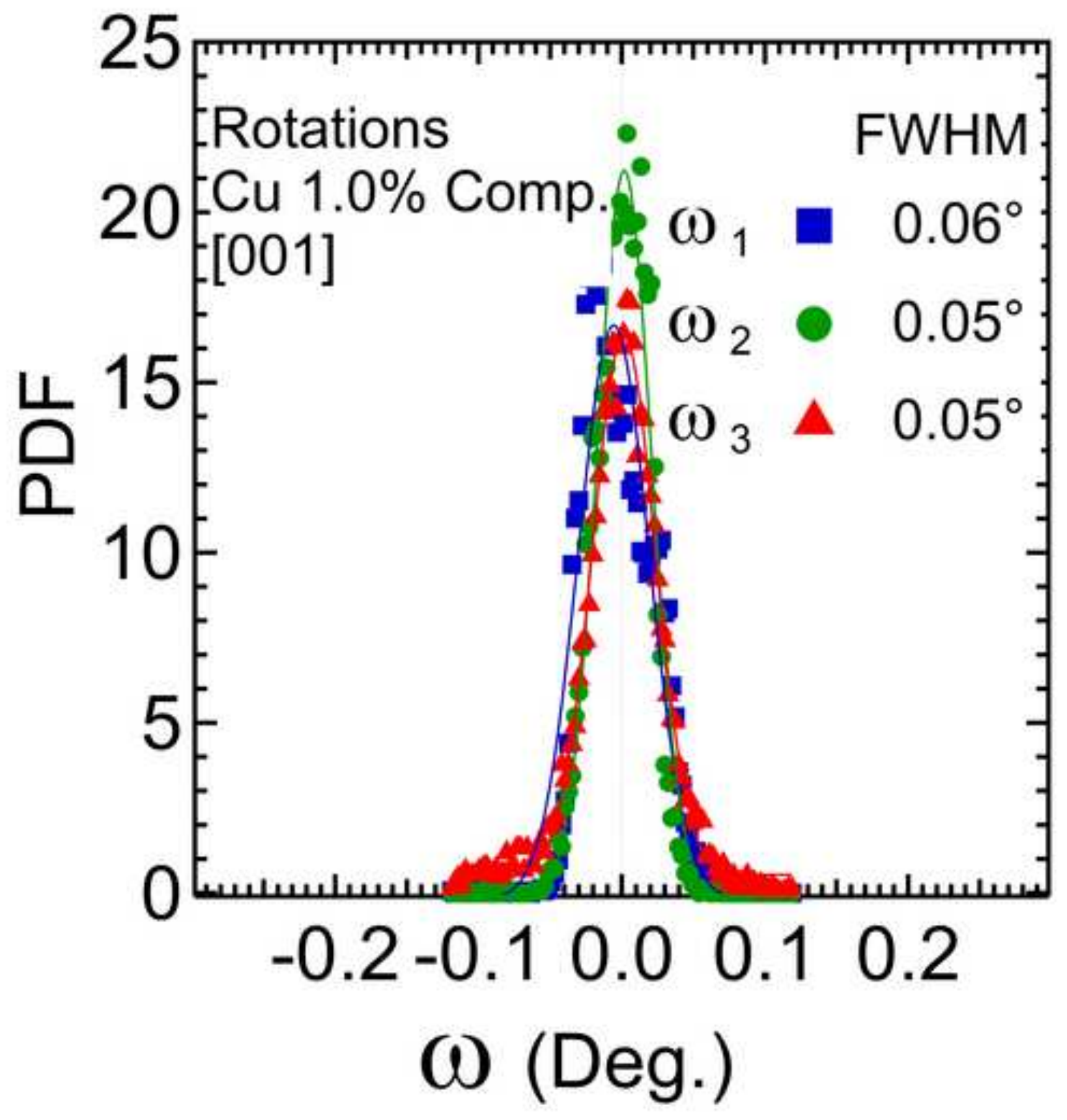

20:

$$
\begin{gathered}
-0.2-0.10 .00 .10 .2 \\
\omega \text { (Deg.) }
\end{gathered}
$$




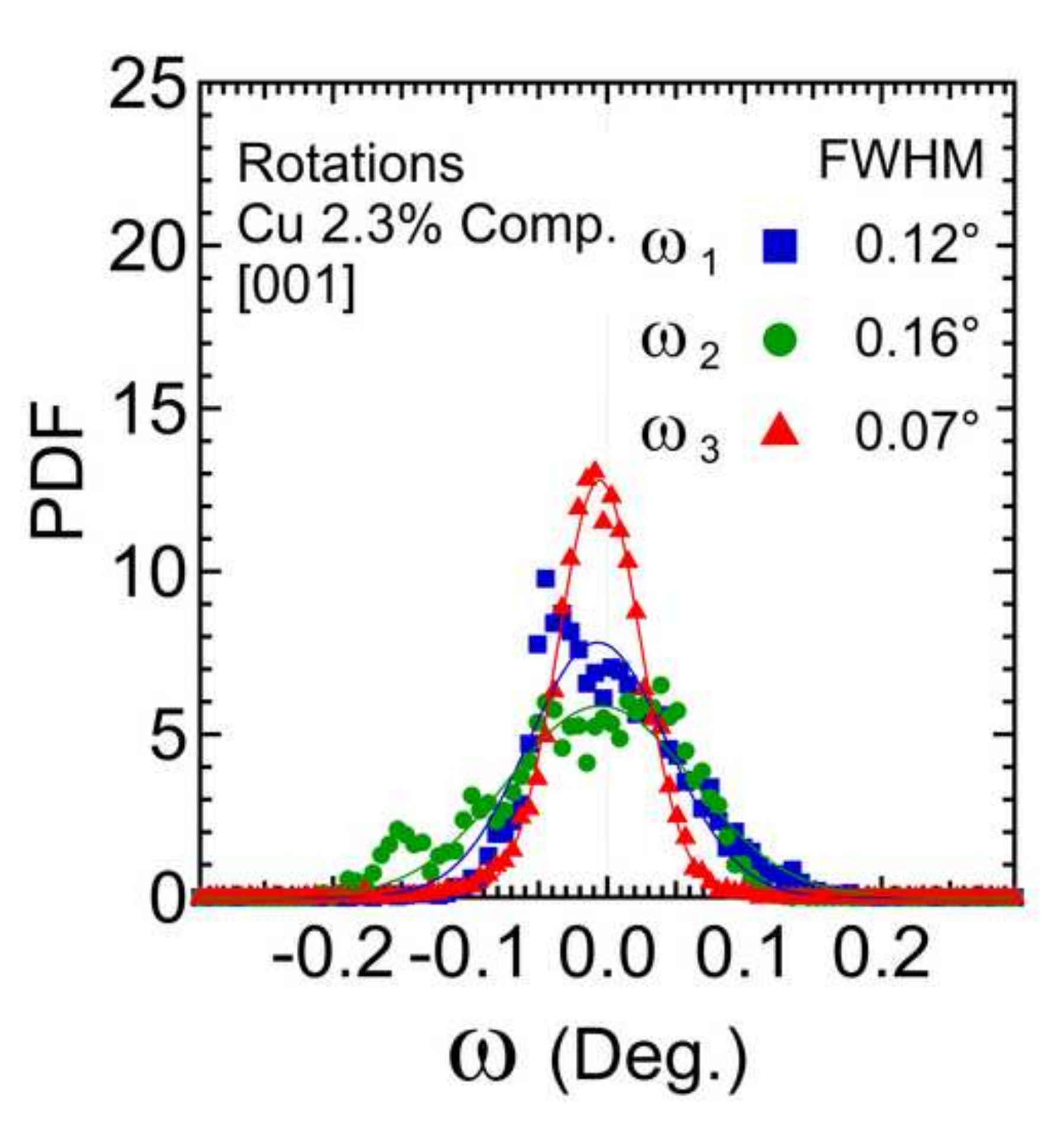

Figure_05b_color

\footnotetext{
列
}
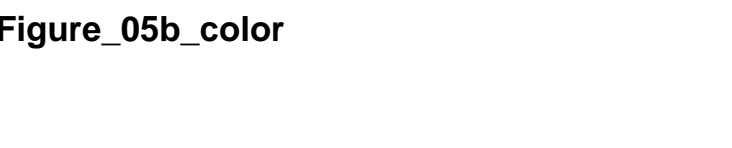

$$
\begin{array}{r}
-0.2-0.10 .00 .1 \\
\omega \text { (Deg.) }
\end{array}
$$

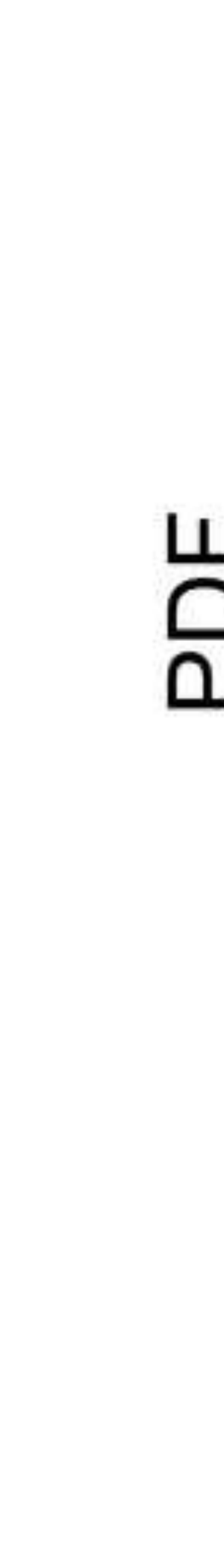


Figure_06a (prefered to be color in printed version)
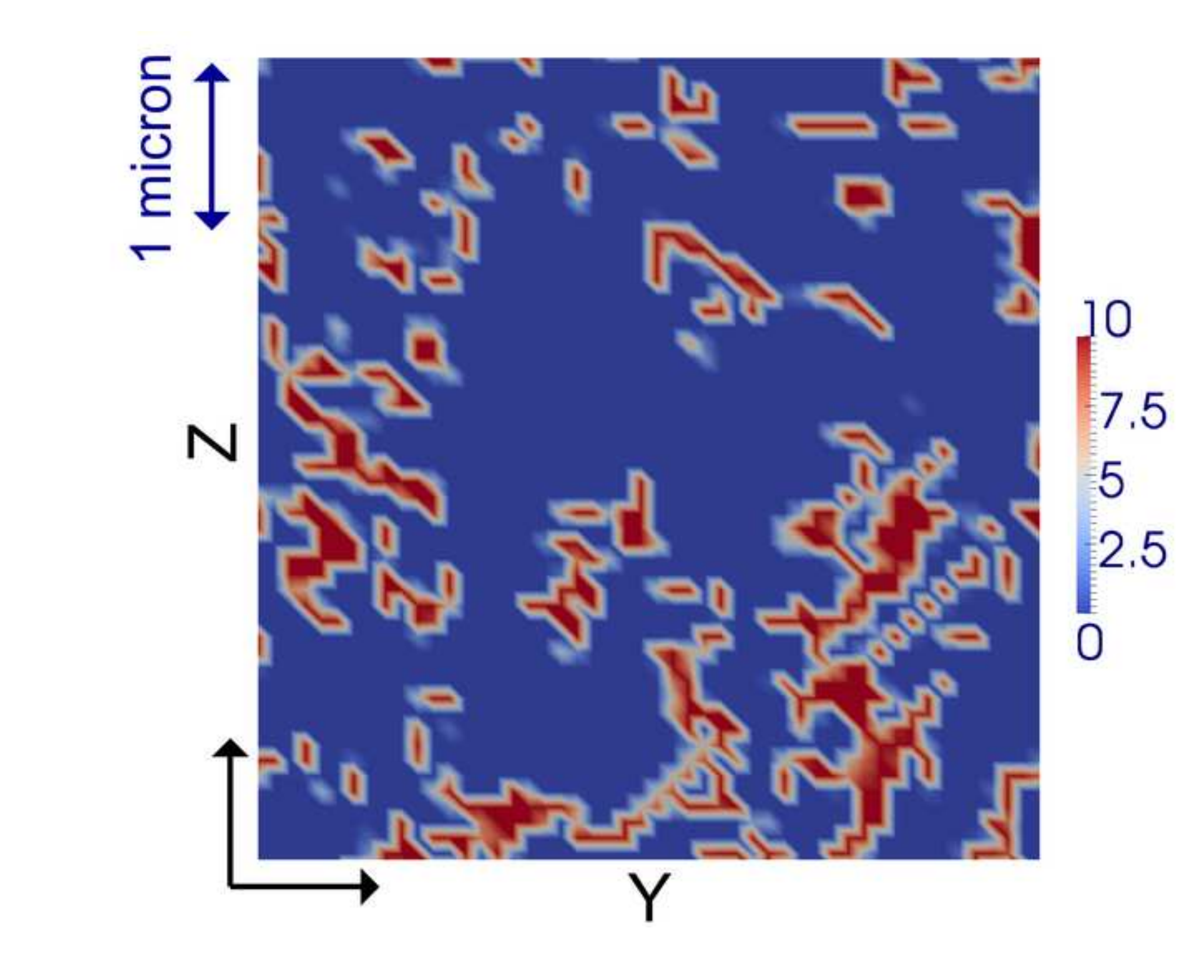

$$
\text { (1) }
$$
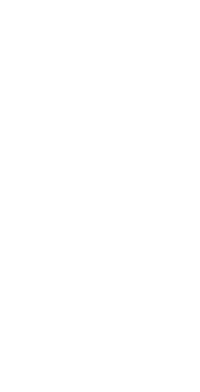


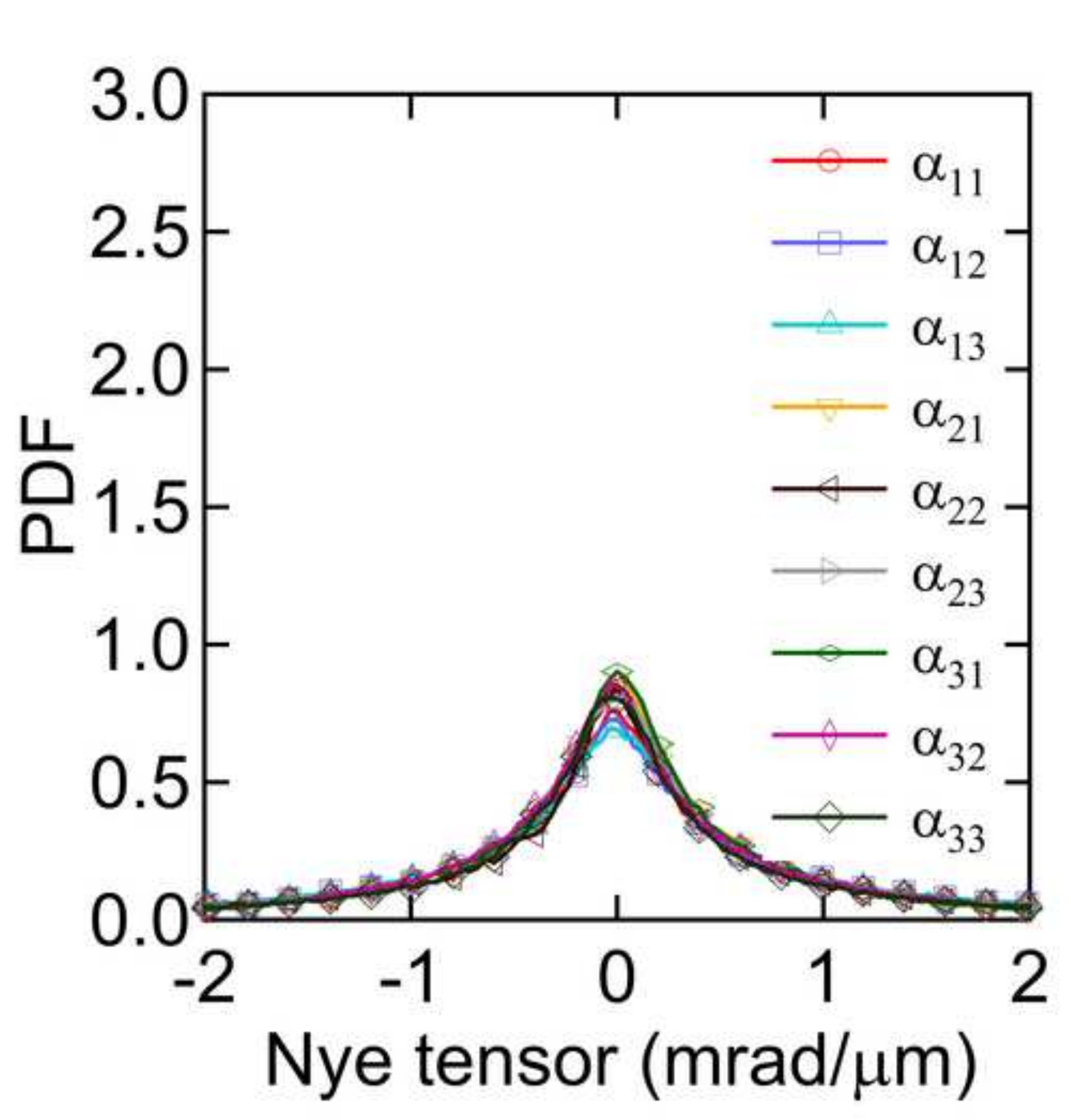

Figure_07b_color 
Figure_09

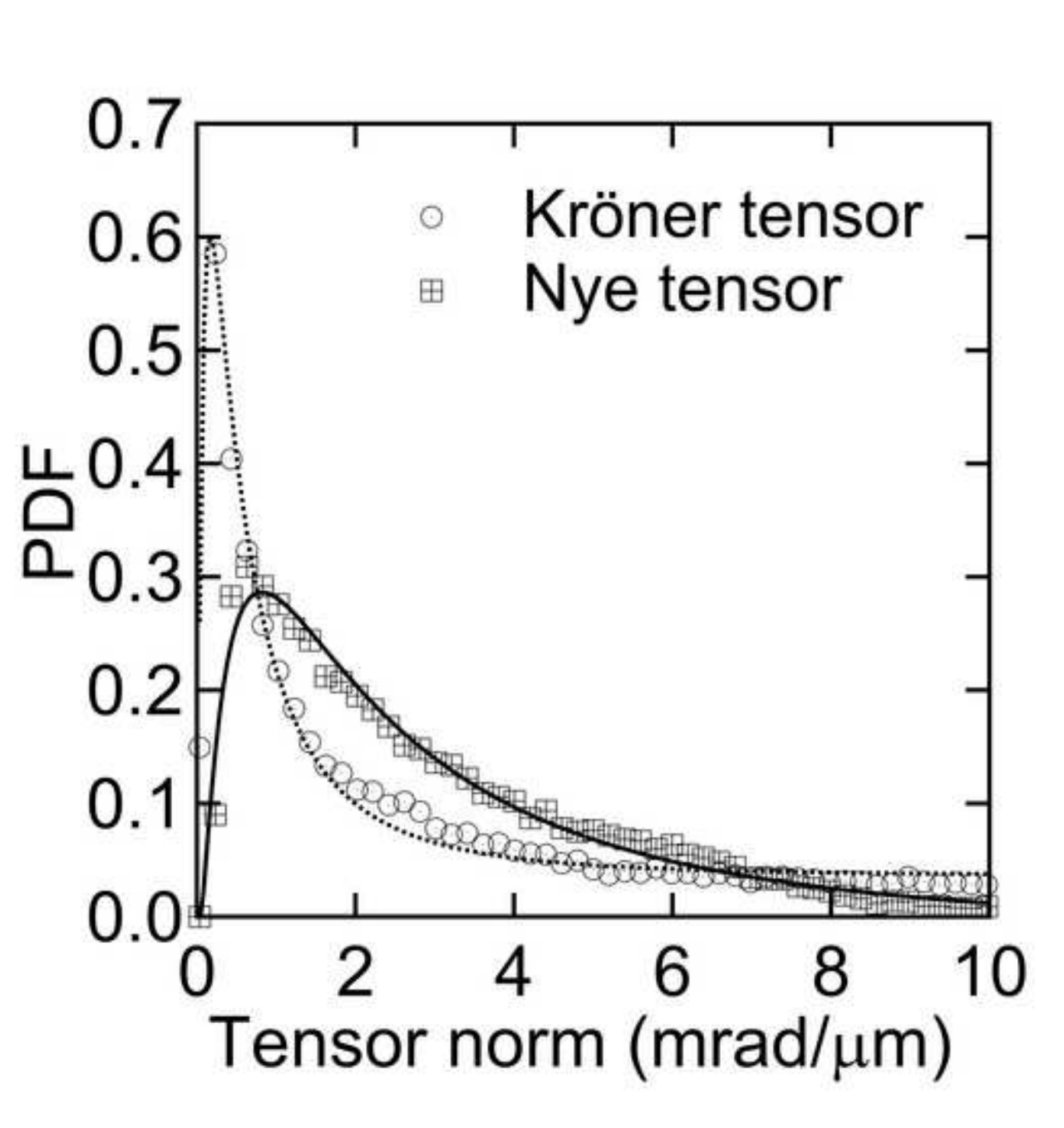

Figure 09
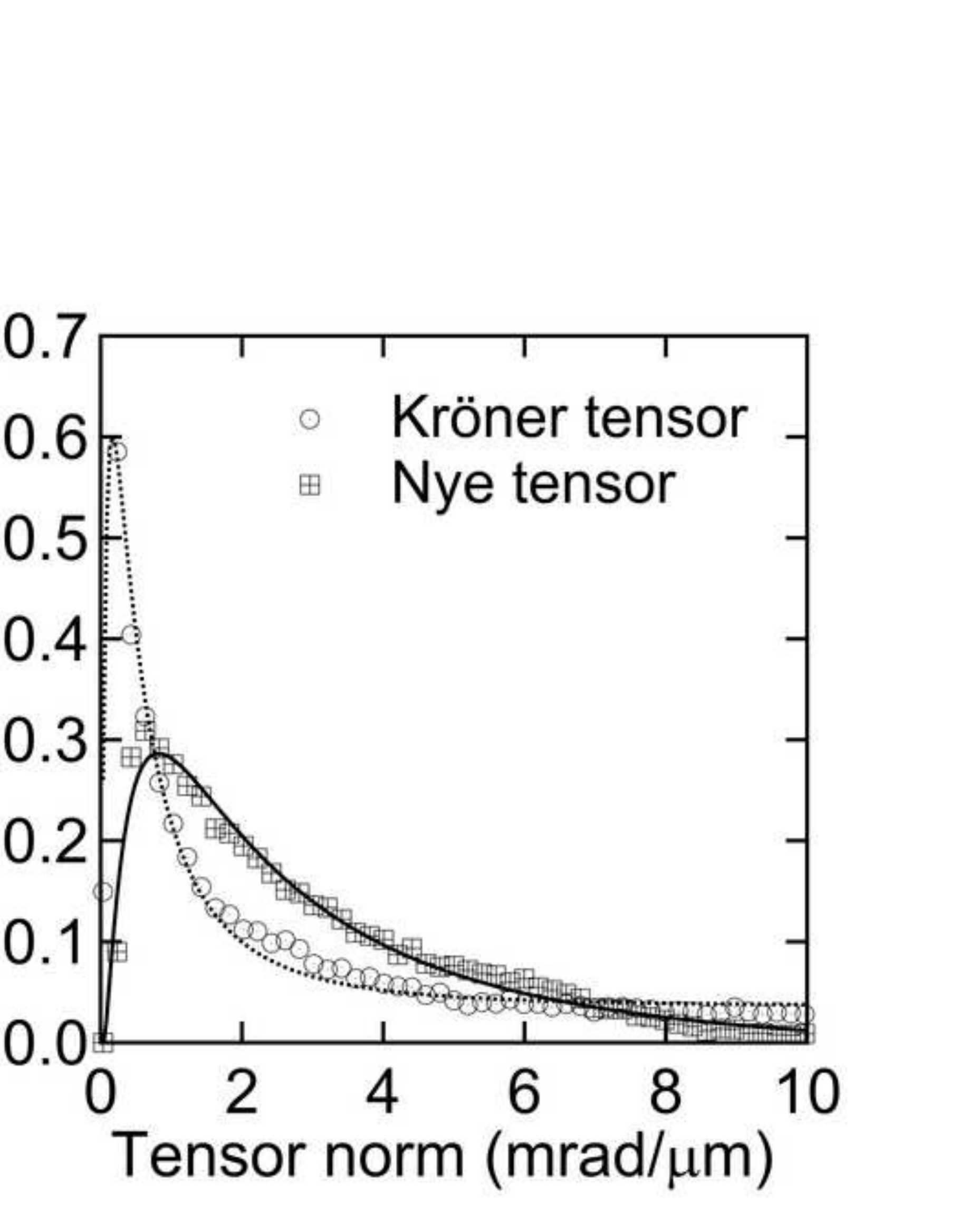


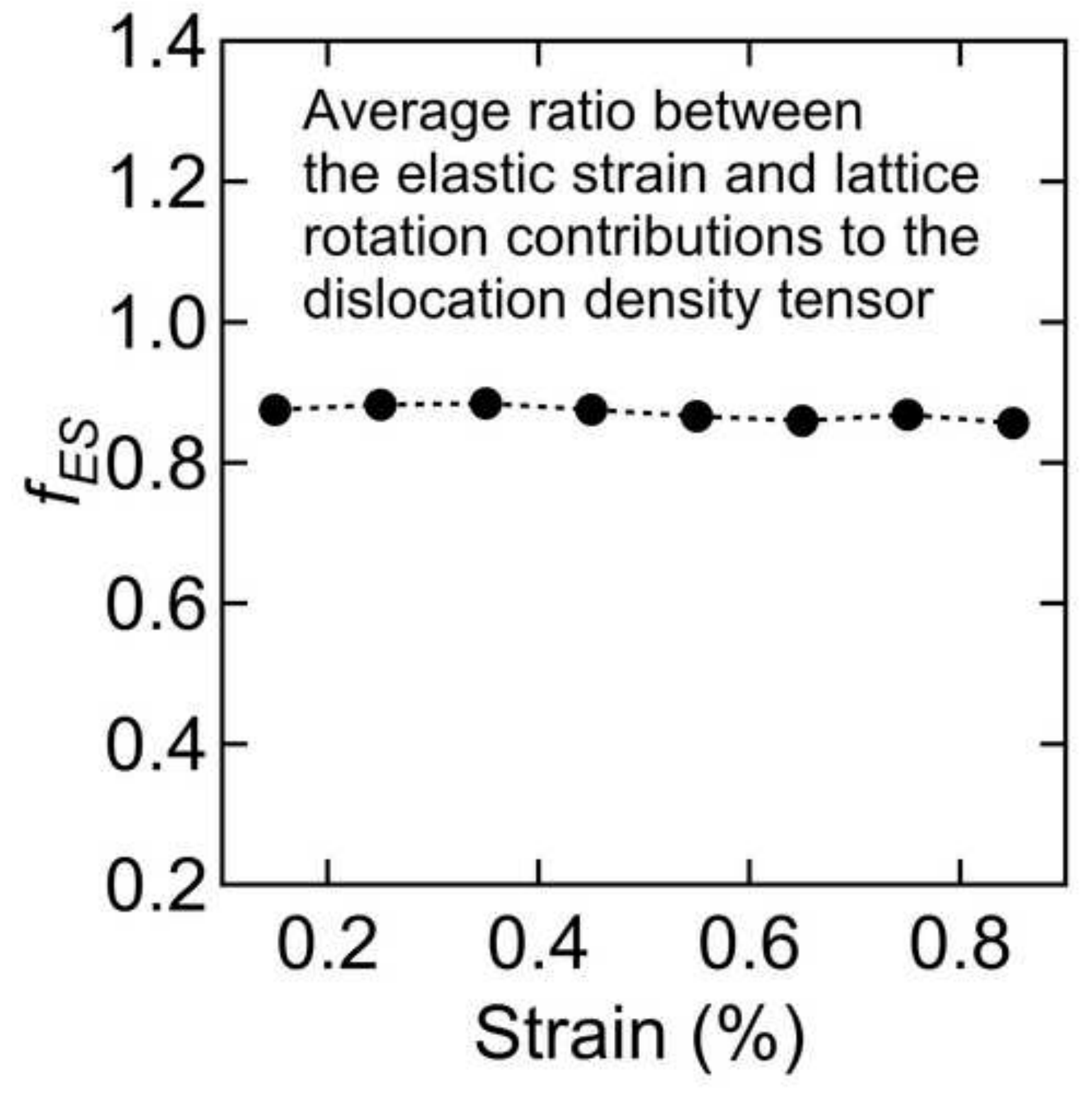




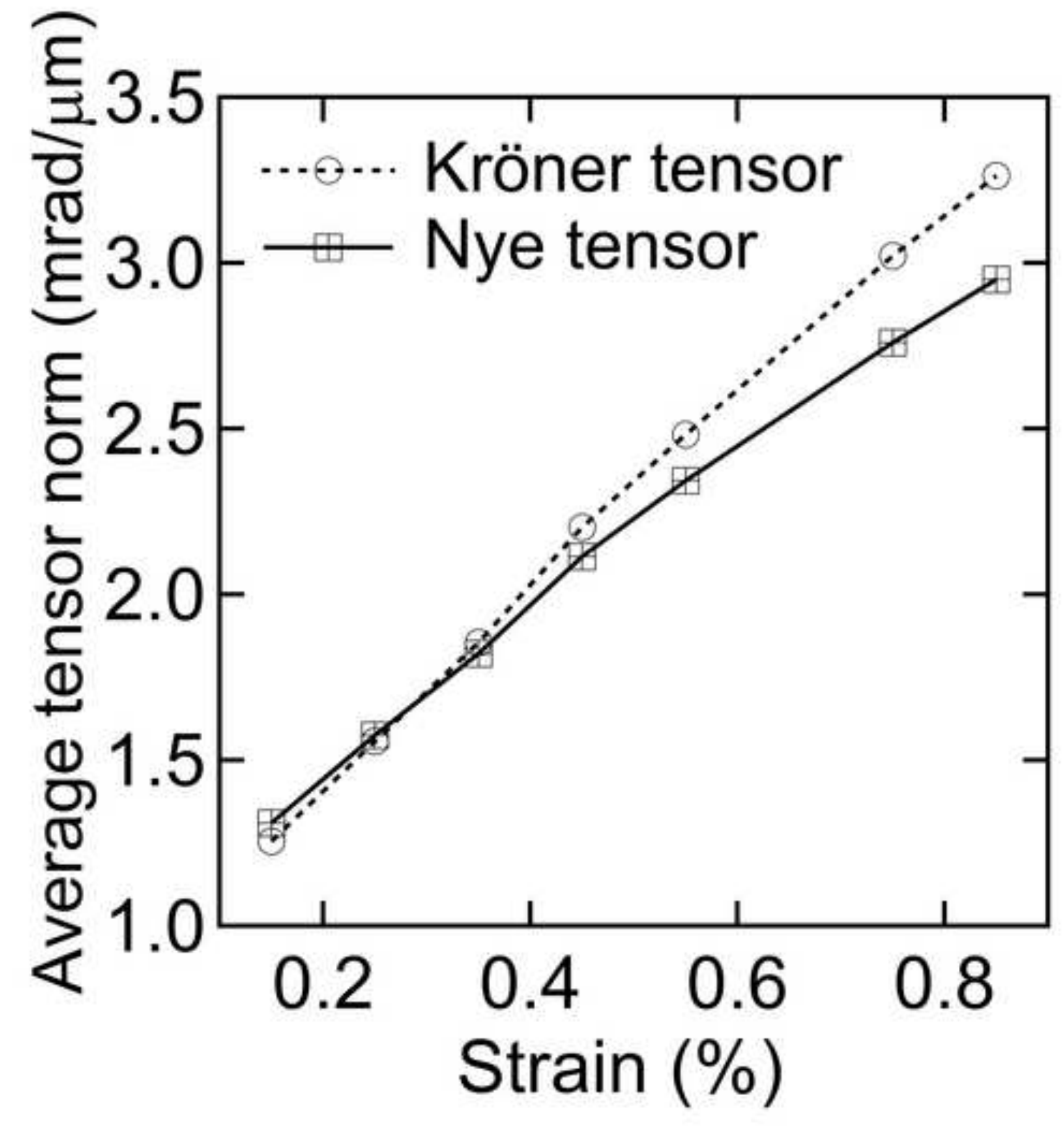

Figure_10b 


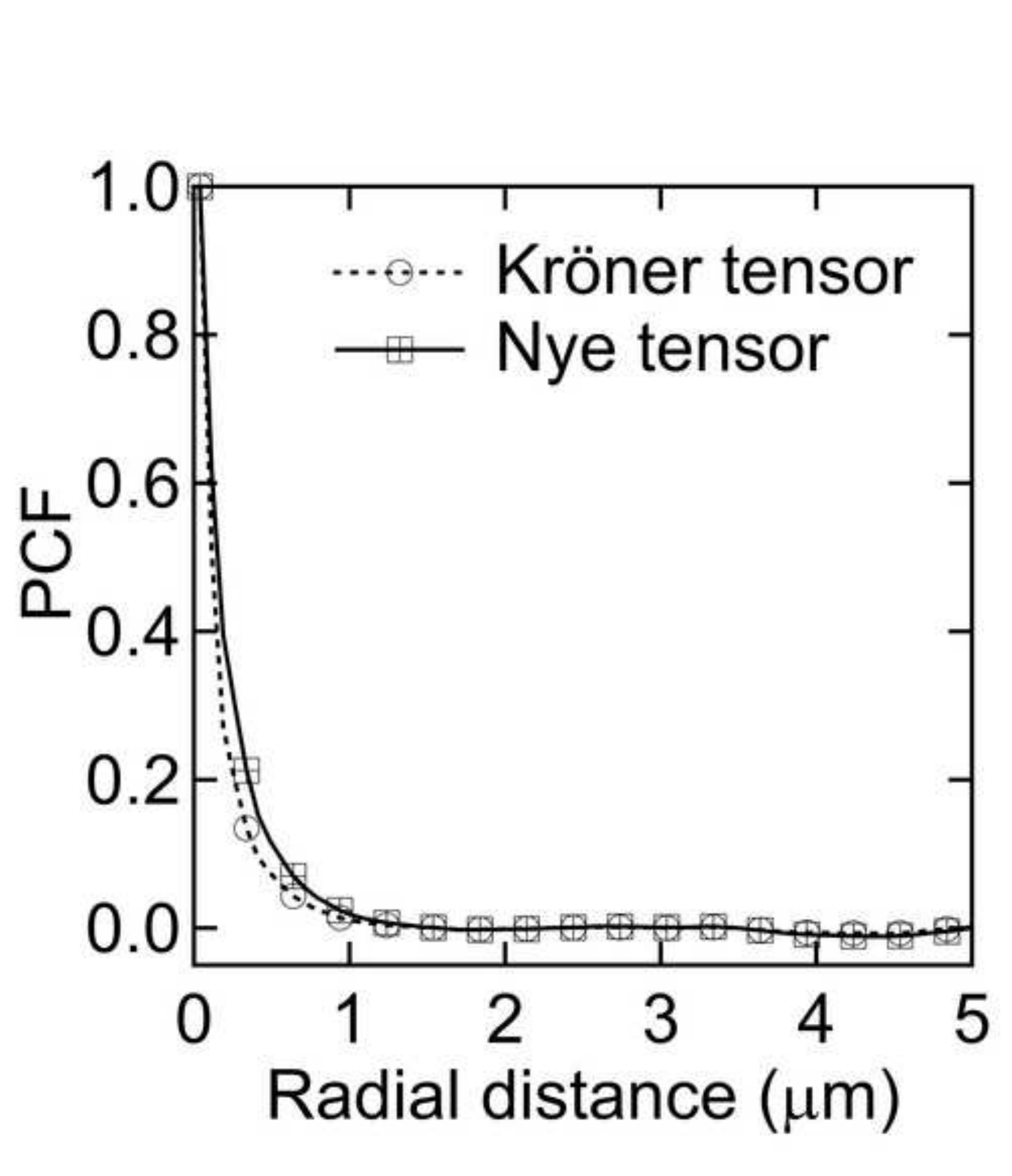

Figure_11

\begin{abstract}
-

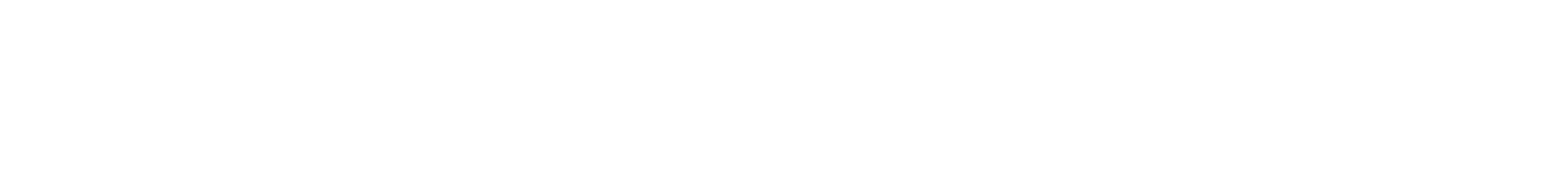

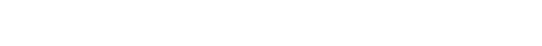
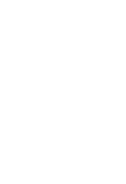

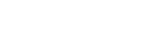

(

?

C

( 


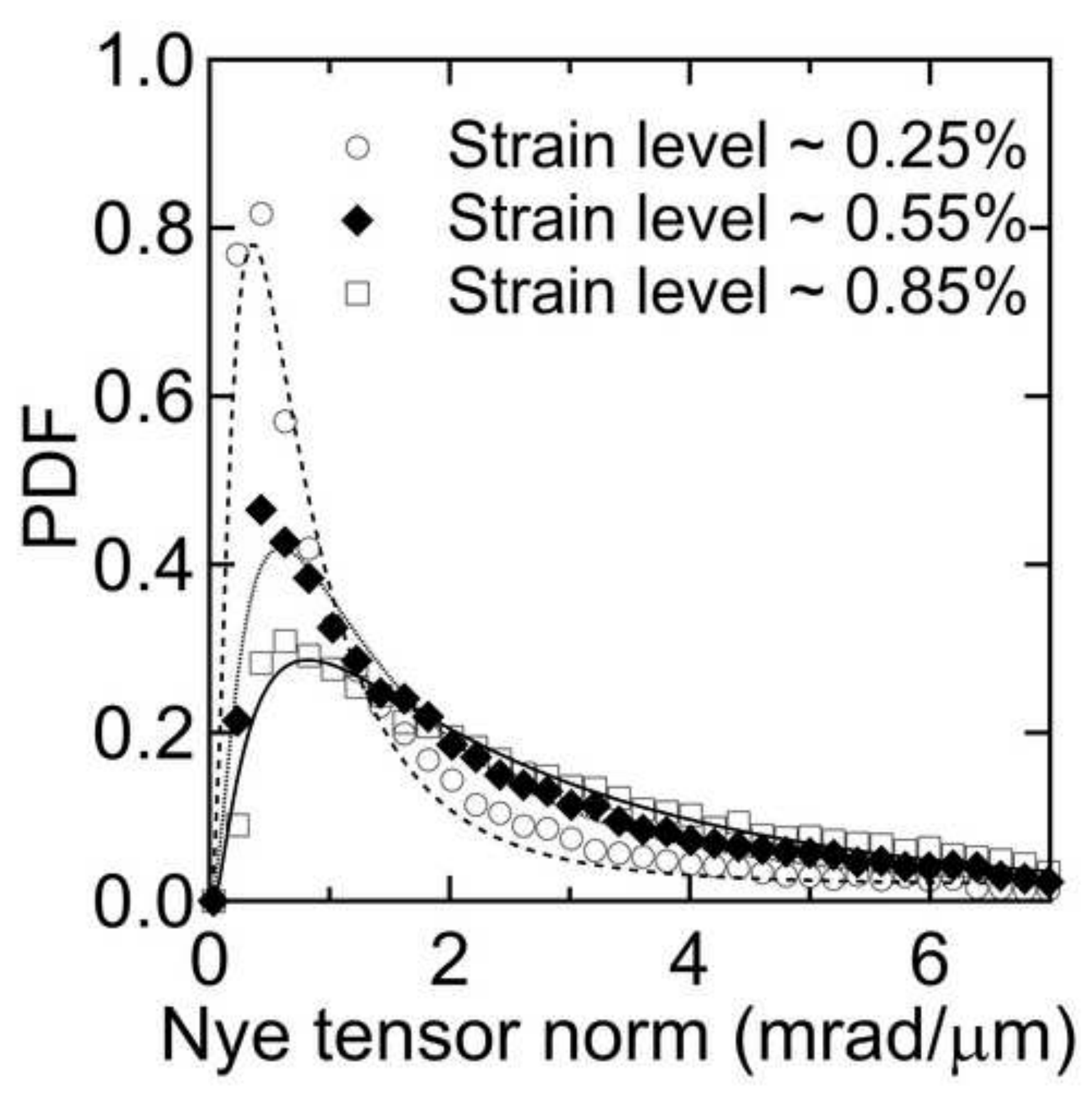

Figure_12a 


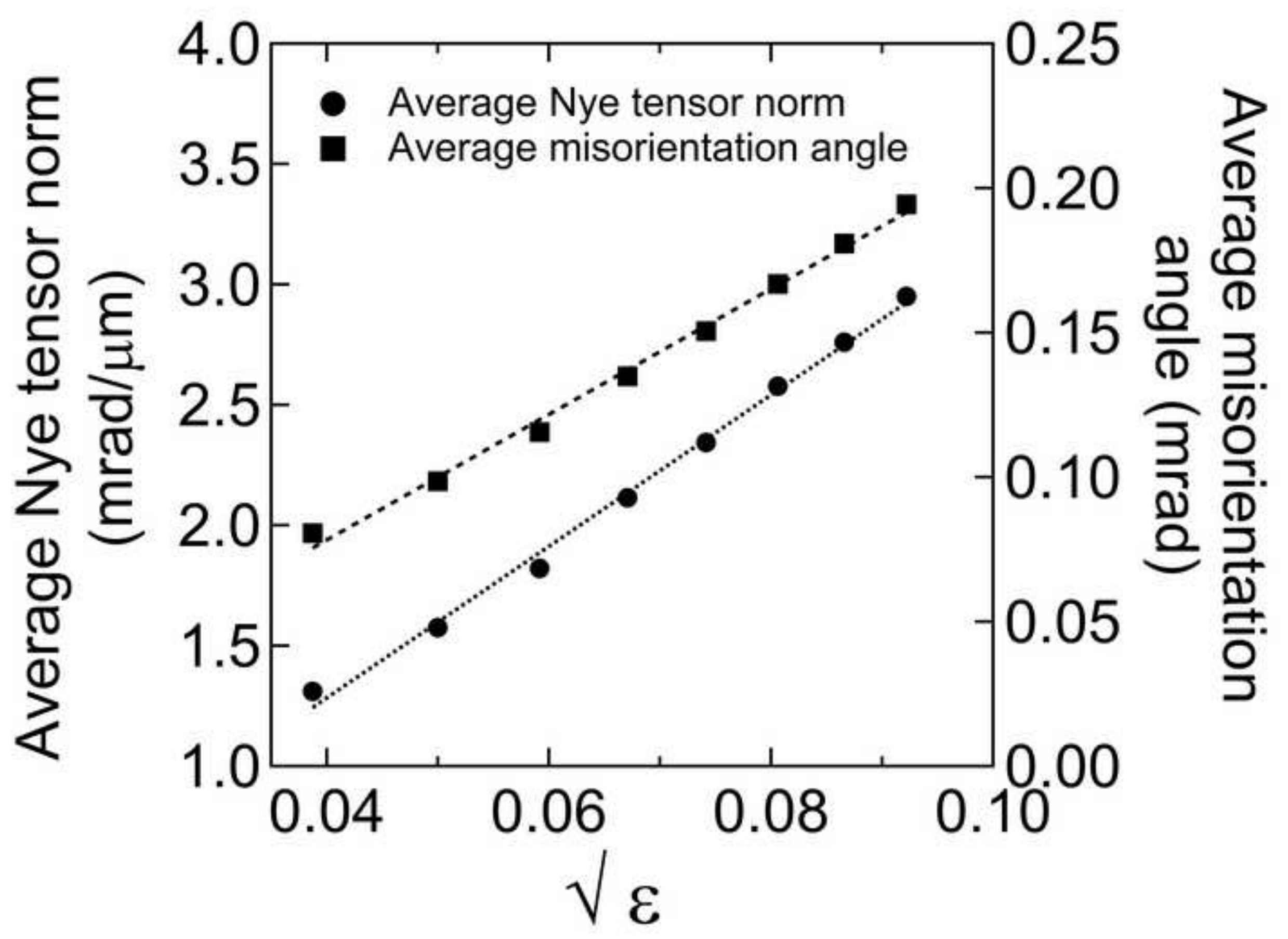




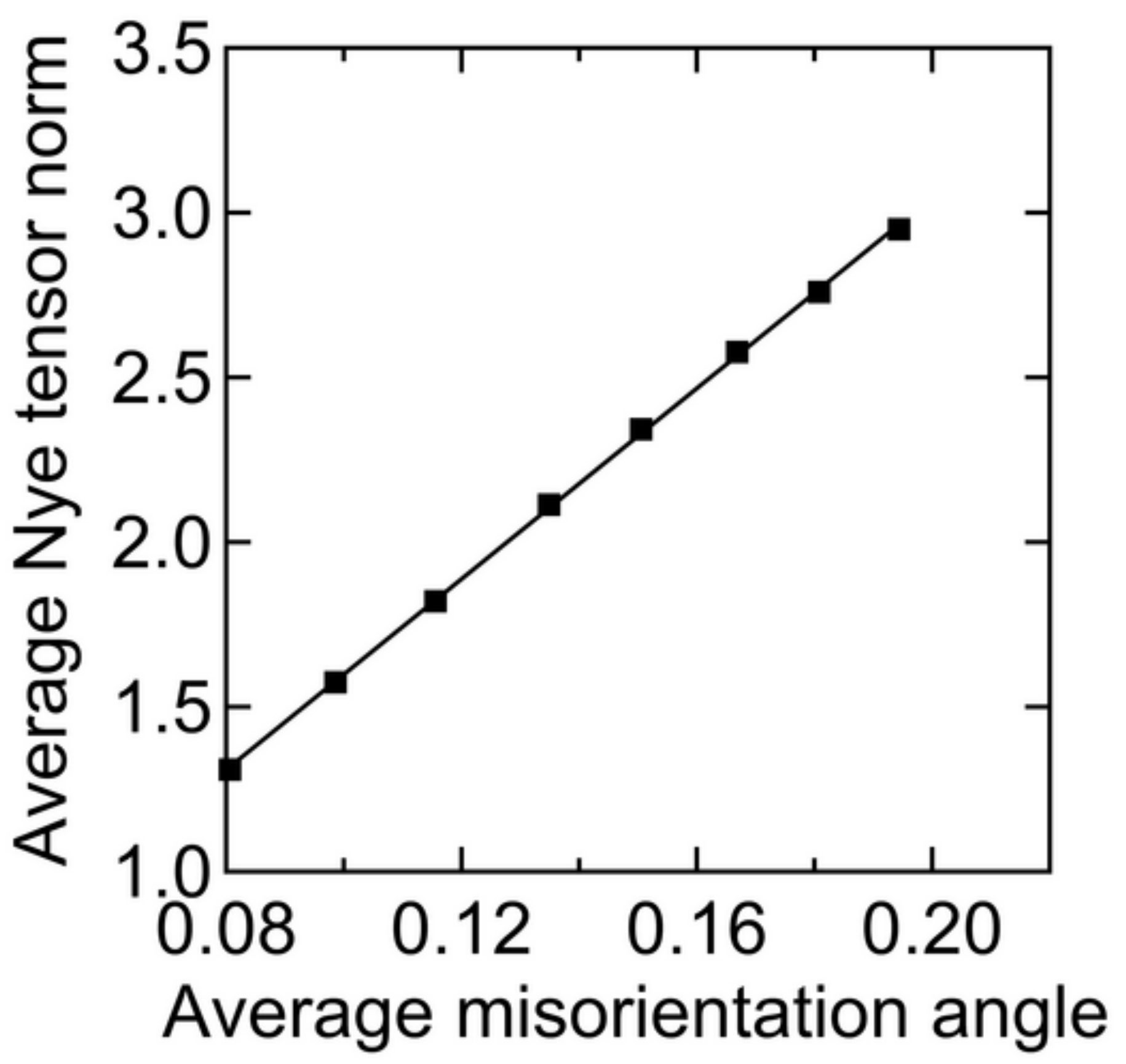


Figure_01a_blackwhite

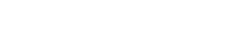

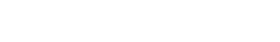

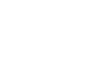
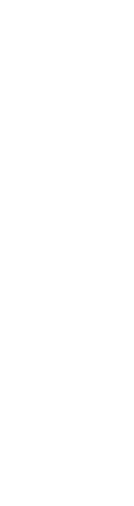


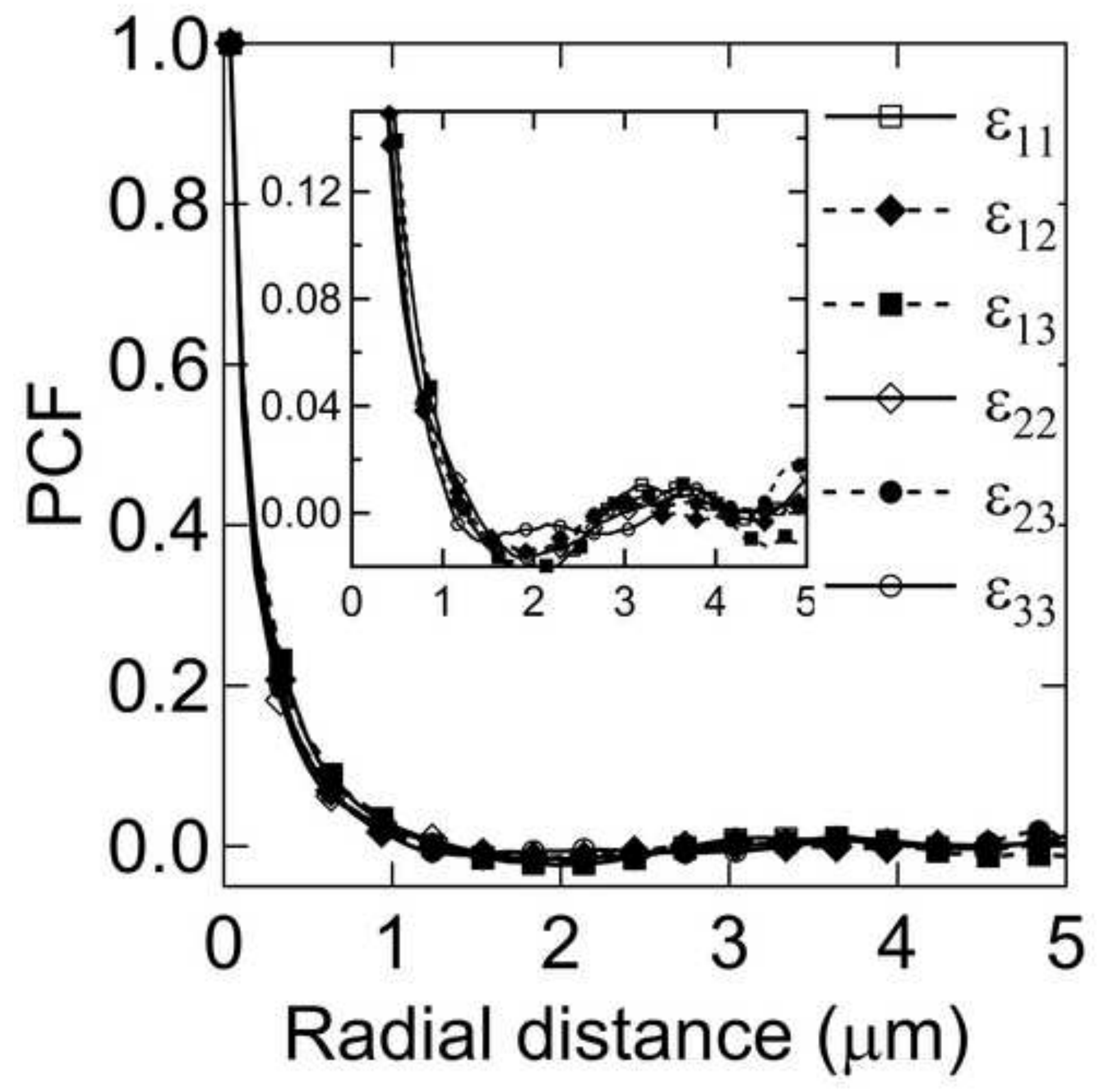

Figure_01b_blackwhite 


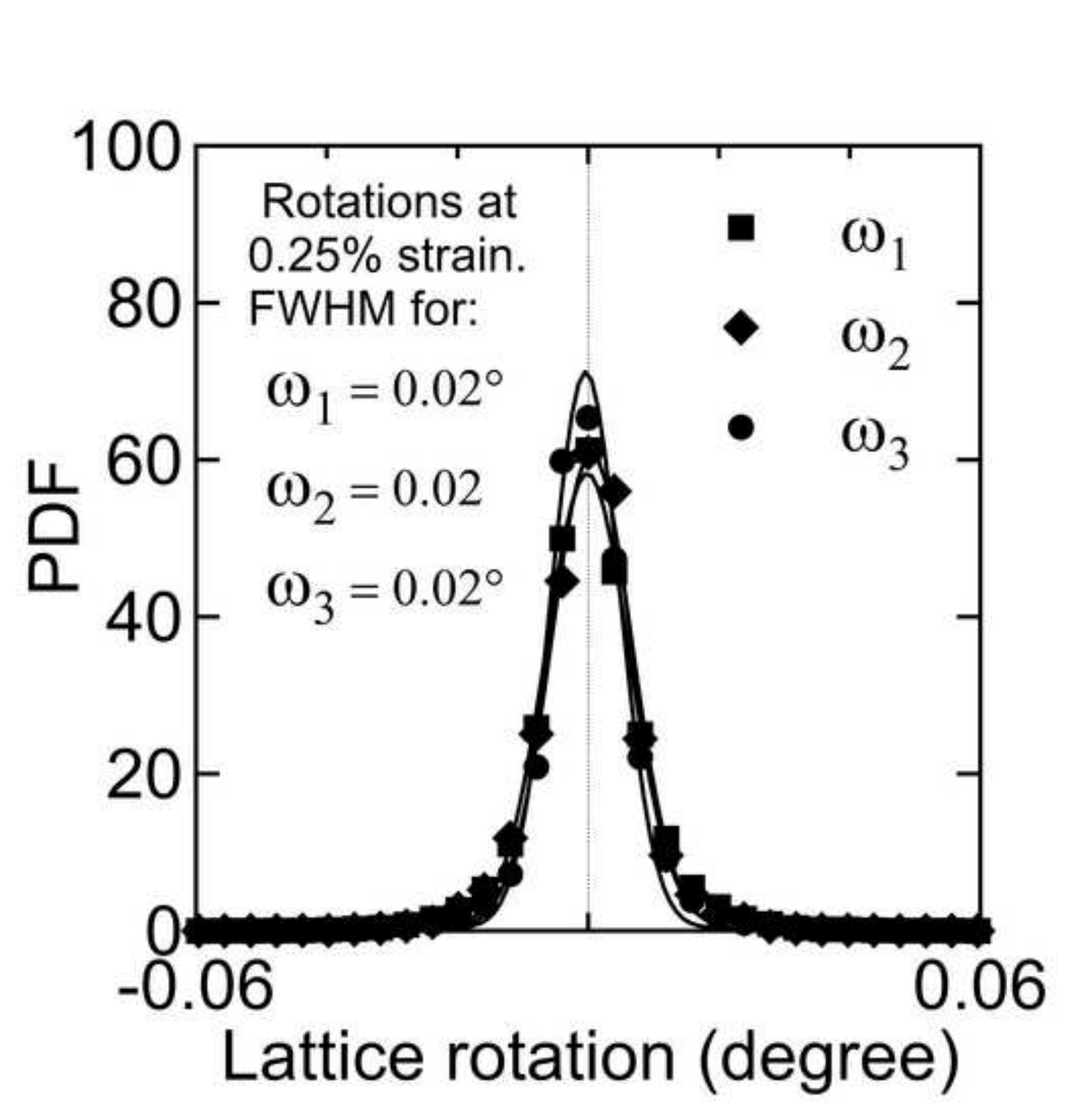

Figure_02a_blackwhite

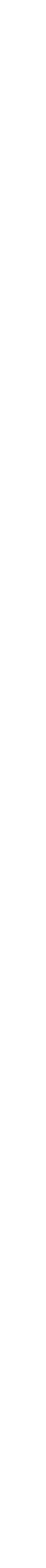

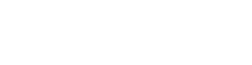

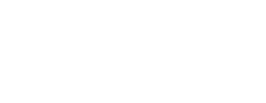

(
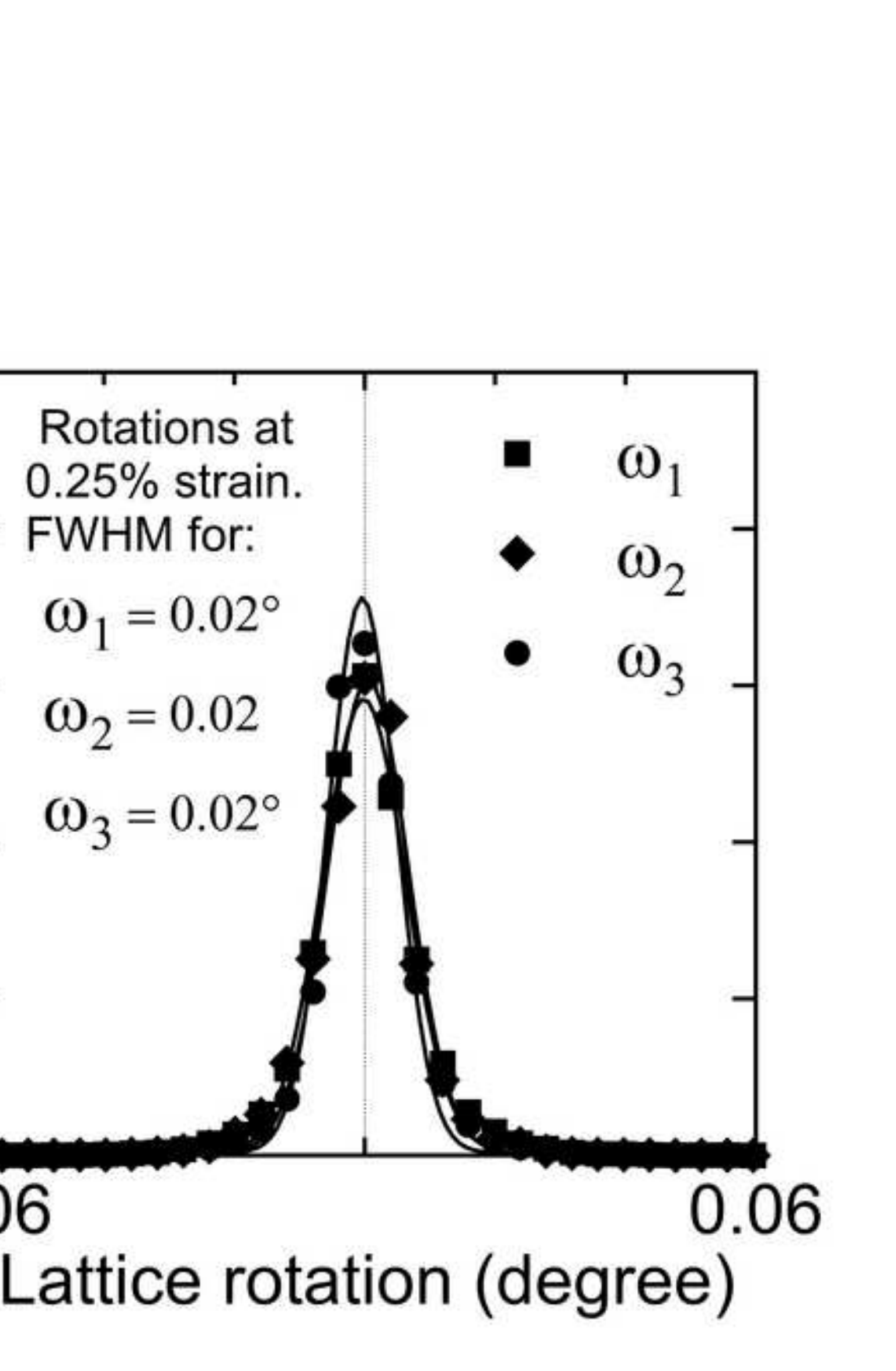
Figure_02b_blackwhite

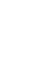

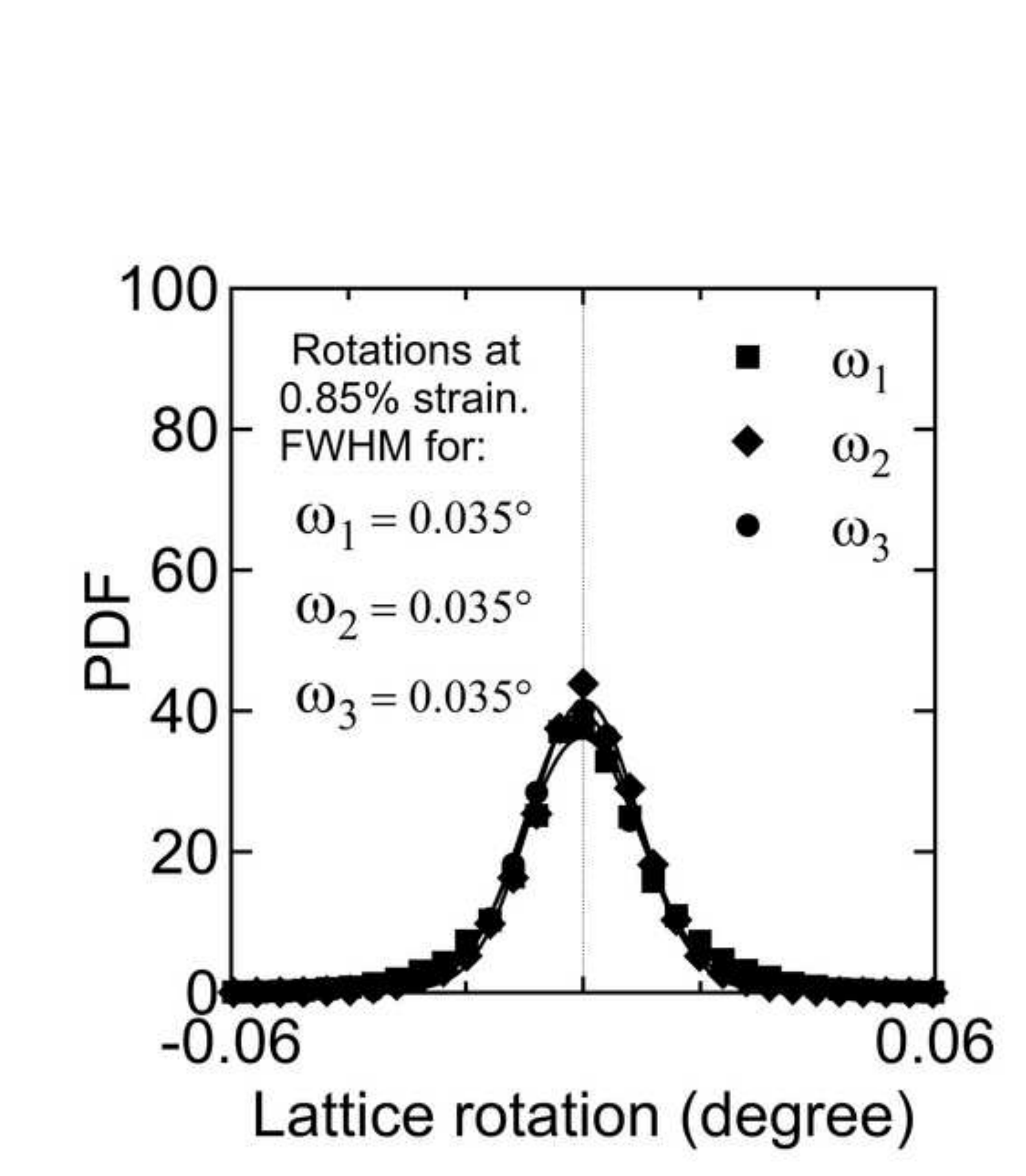

O

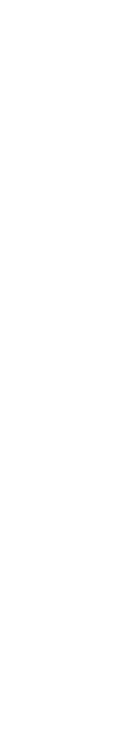




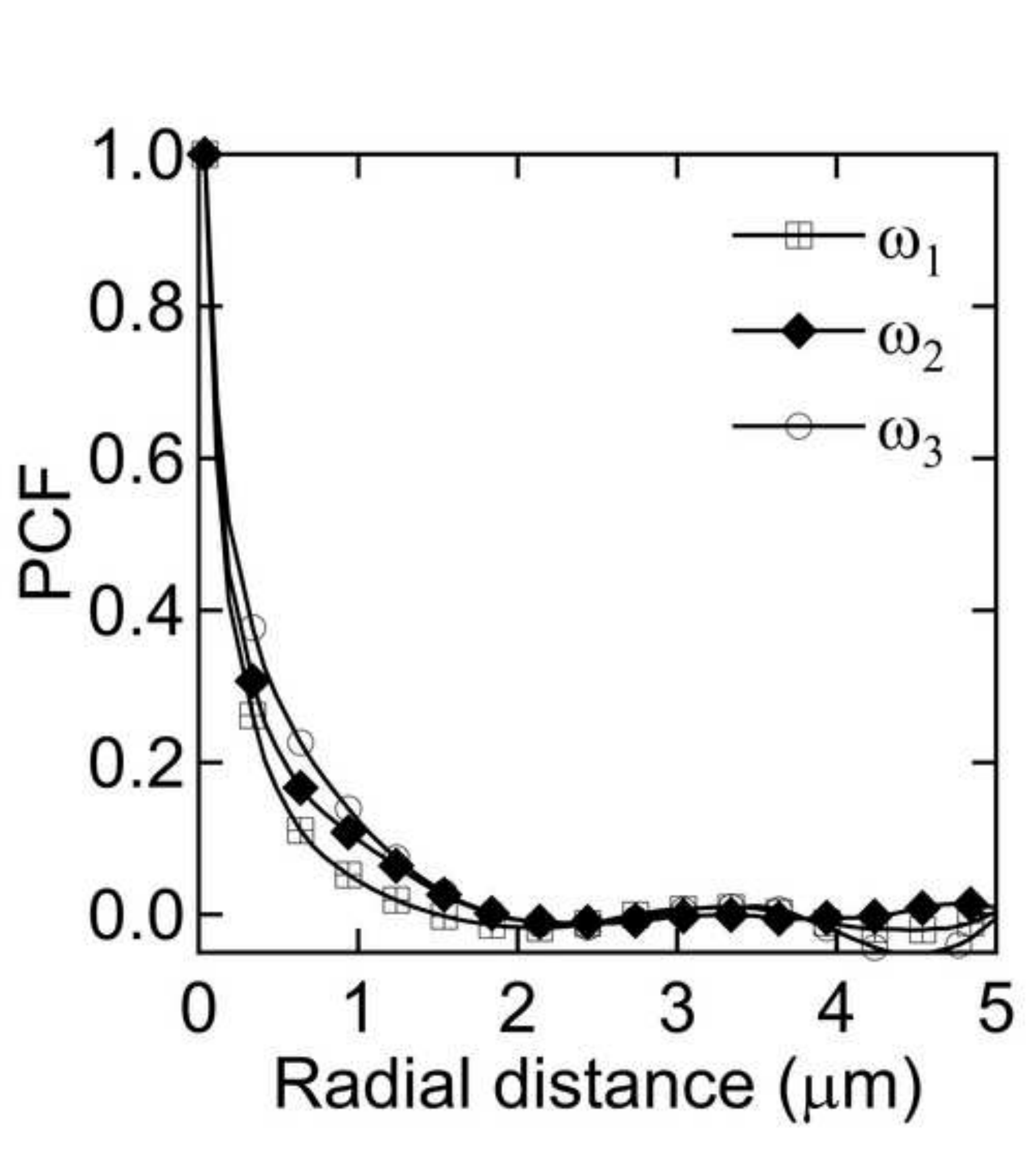

Figure_02c_blackwhite

Figure_02c_blackwhite

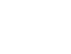

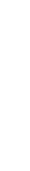
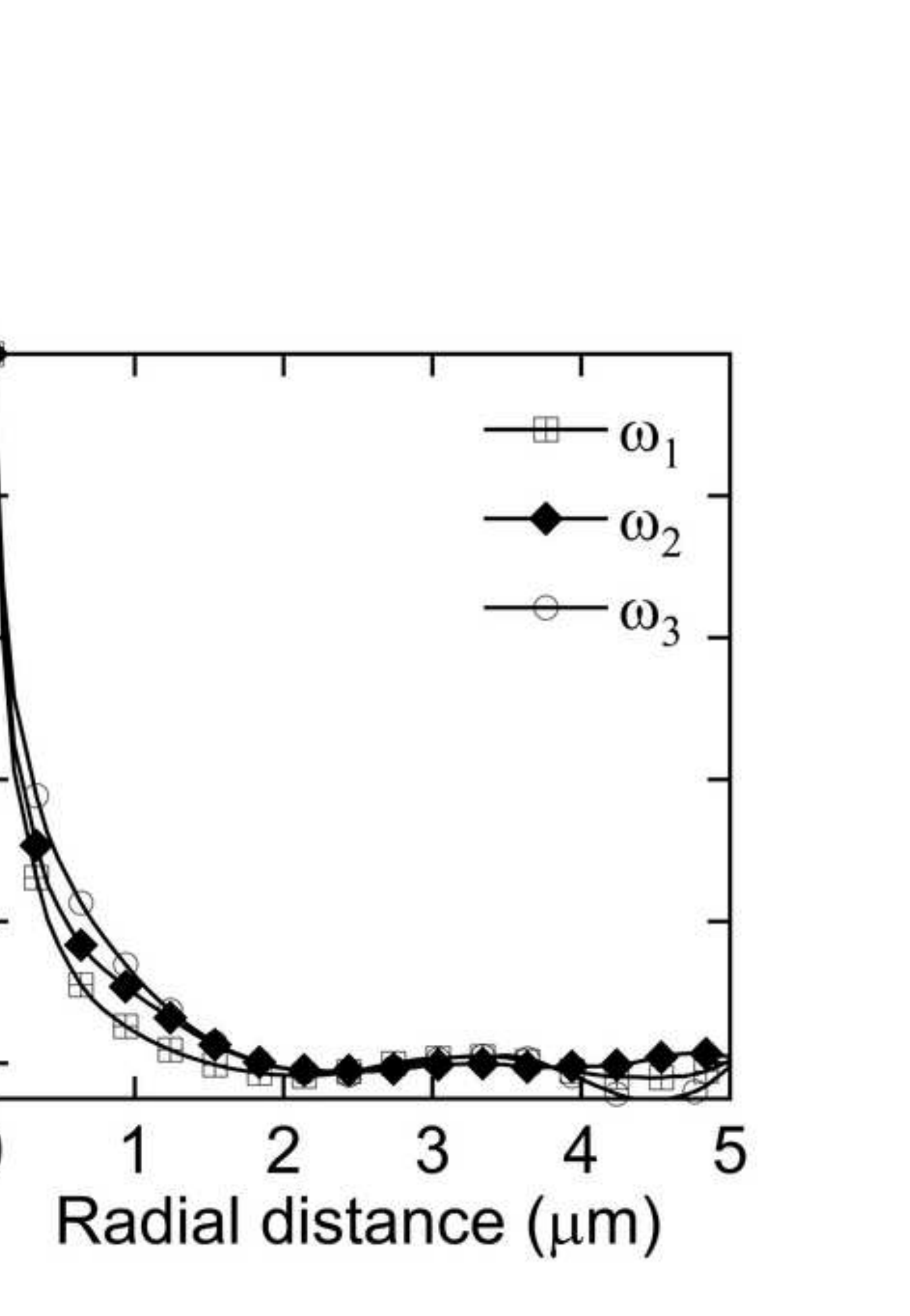

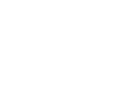




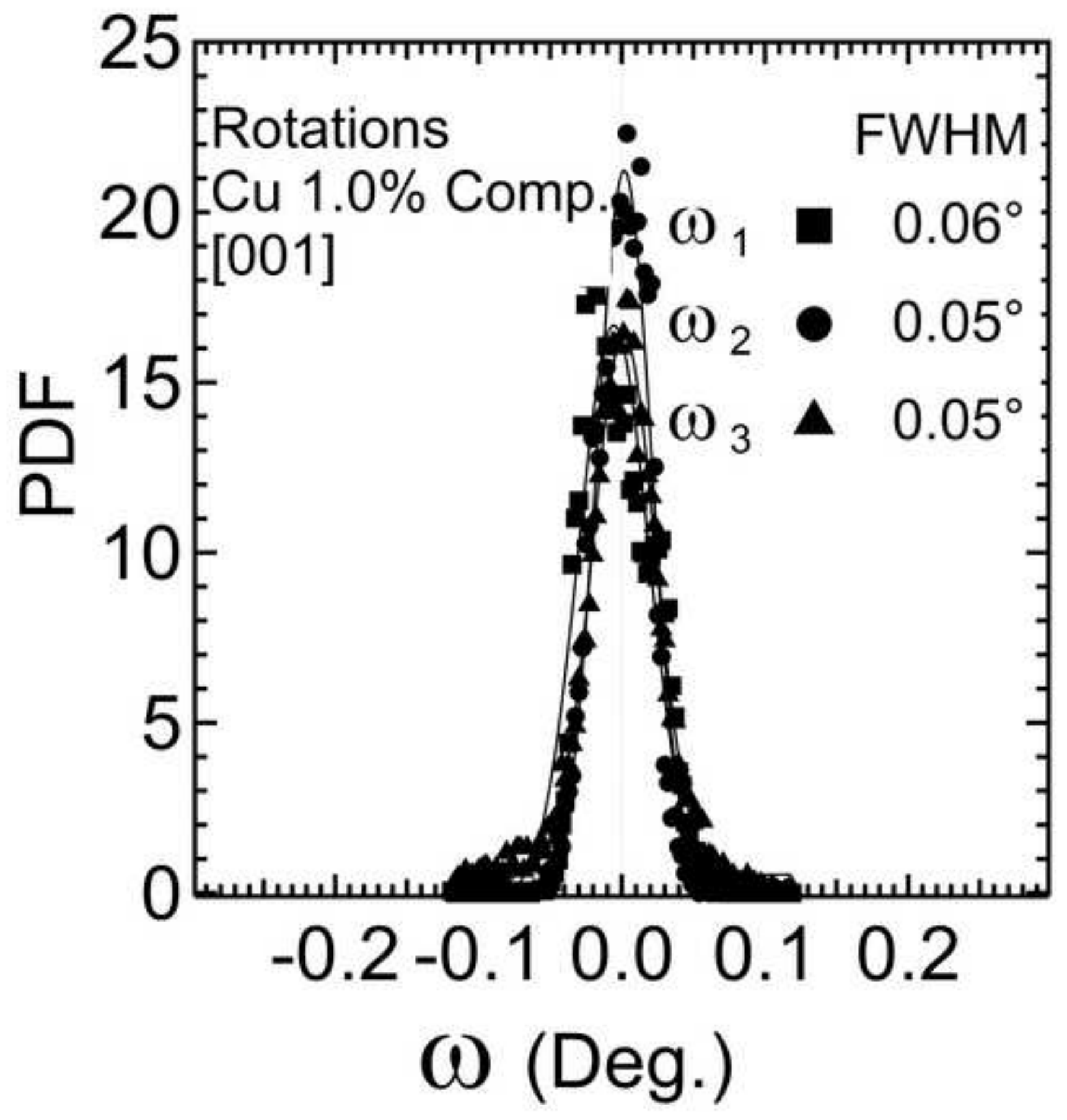

(1)

$$
\begin{gathered}
-0.2-0.10 .00 .10 .2 \\
\omega \text { (Deg.) }
\end{gathered}
$$




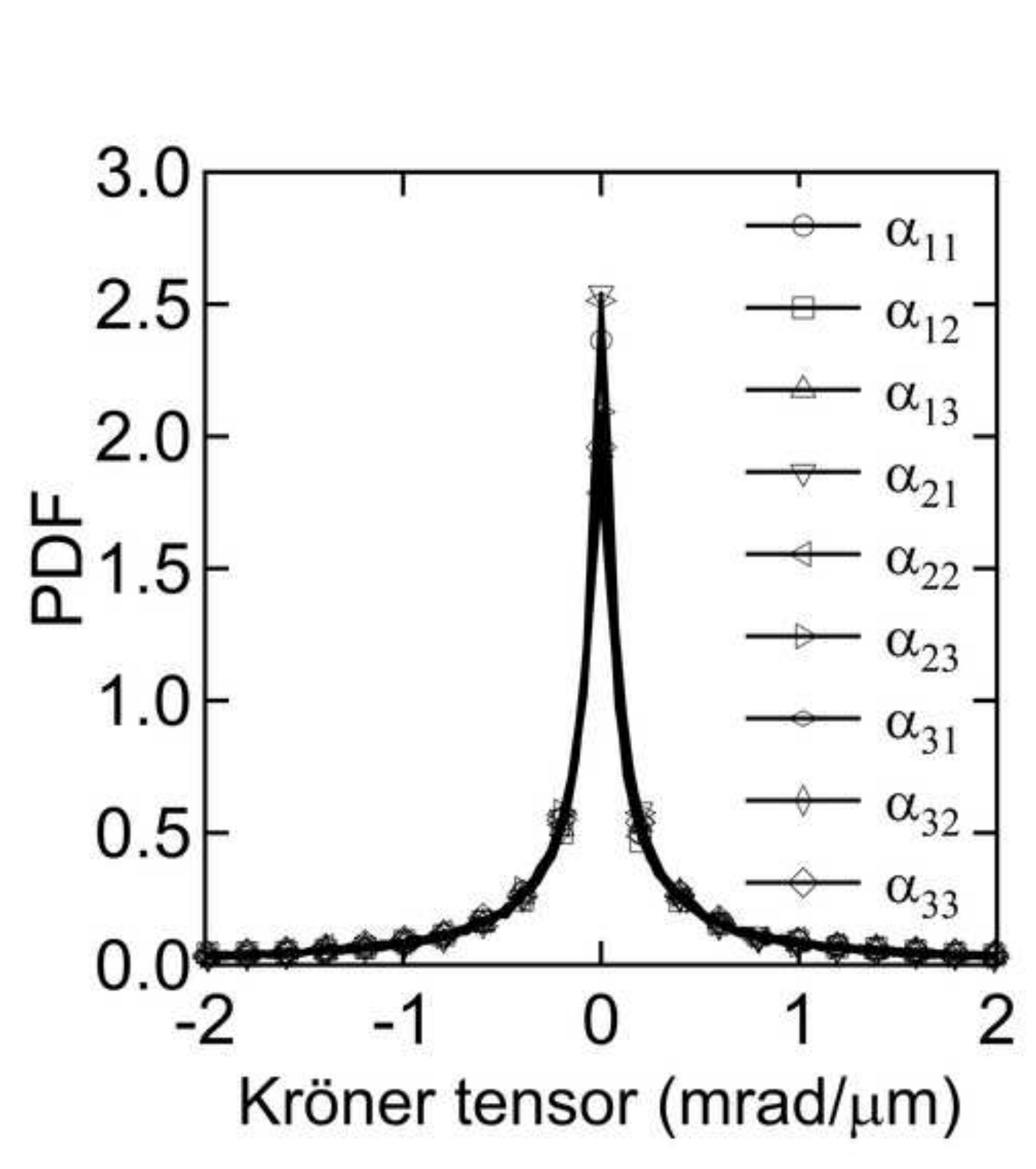

Figure_07a_blackwhite

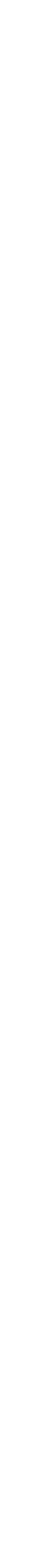
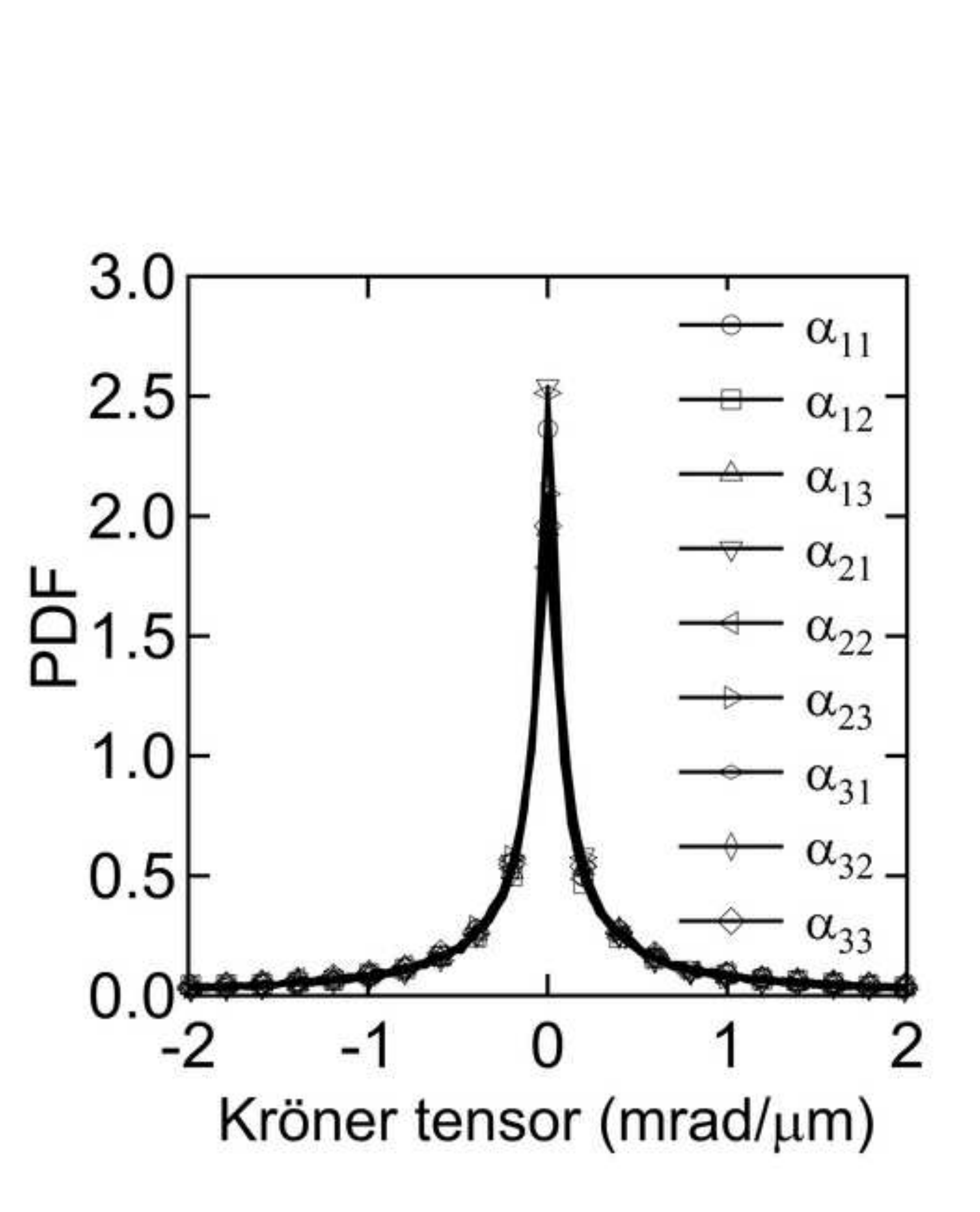


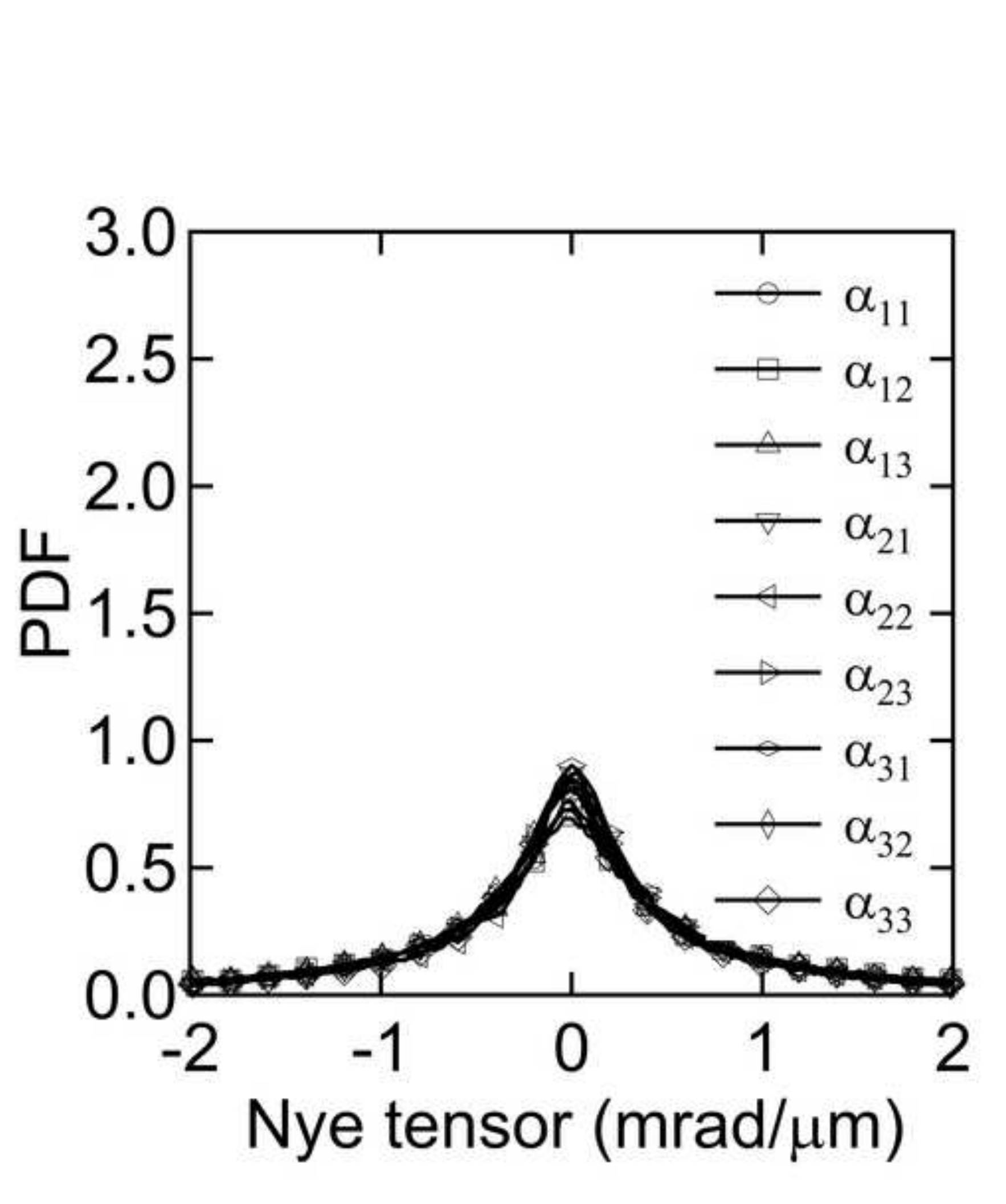

Figure_07b_blackwhite

\author{
Figure_07b_blackwhite_
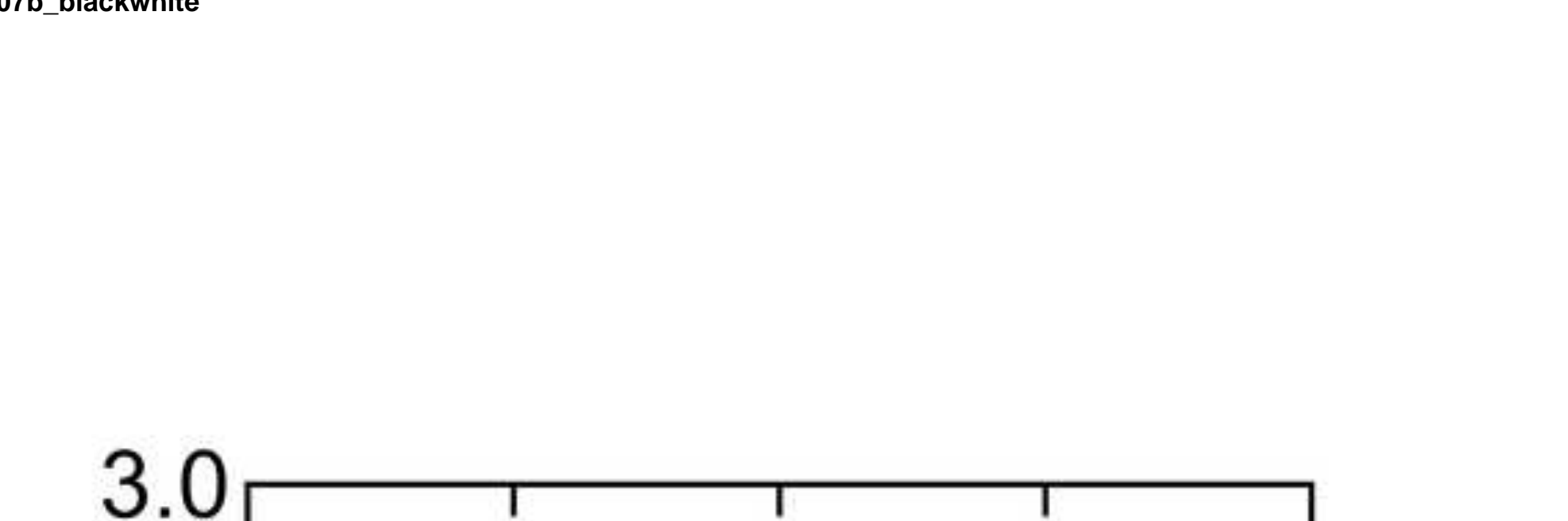

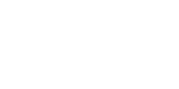

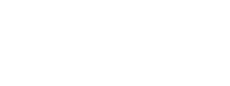

